\title{
An Algorithmic Proof of the Lovász Local Lemma via Resampling Oracles
}

\author{
Nicholas J. A. Harvey \\ University of British Columbia \\ Vancouver, Canada \\ nickhar@cs. ubc.ca
}

\author{
Jan Vondrák \\ IBM Almaden Research Center \\ San Jose, CA, USA \\ jvondrakeus.ibm.com
}

\begin{abstract}
The Lovász Local Lemma is a seminal result in probabilistic combinatorics. It gives a sufficient condition on a probability space and a collection of events for the existence of an outcome that simultaneously avoids all of those events. Finding such an outcome by an efficient algorithm has been an active research topic for decades. Breakthrough work of Moser and Tardos (2009) presented an efficient algorithm for a general setting primarily characterized by a product structure on the probability space.

In this work we present an efficient algorithm for a much more general setting. Our main assumption is that there exist certain functions, called resampling oracles, that can be invoked to address the undesired occurrence of the events. We show that, in all scenarios to which the original Lovász Local Lemma applies, there exist resampling oracles, although they are not necessarily efficient. Nevertheless, for essentially all known applications of the Lovász Local Lemma and its generalizations, we have designed efficient resampling oracles. As applications of these techniques, we present new results for packings of Latin transversals, rainbow matchings and rainbow spanning trees.
\end{abstract}




\section{Contents}

1 Introduction 2

1.1 Our contributions $\ldots \ldots \ldots \ldots \ldots \ldots \ldots \ldots$

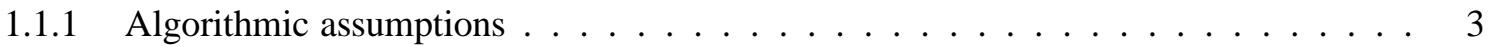

1.2 Our algorithm: MaximalSetResample . . . . . . . . . . . . . . . . . 4

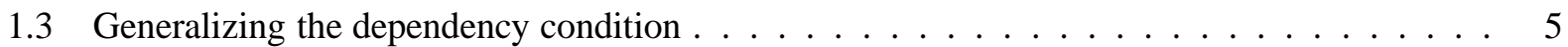

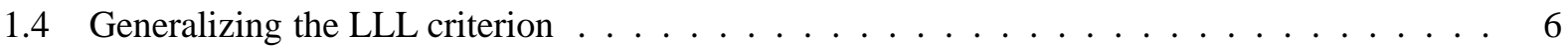

1.5 Techniques and related work $\ldots \ldots \ldots \ldots \ldots \ldots$

2 Resampling oracles: existence and efficiency 9

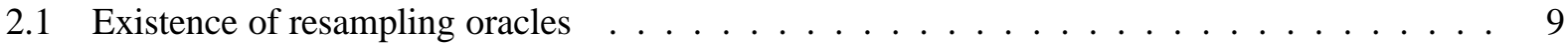

2.1 .1 Example: monotone events on lattices . . . . . . . . . . . . . . 11

2.2 Computational hardness of the LLL . . . . . . . . . . . . . . . . . . . 12

3 Implementation of resampling in specific settings $\quad 13$

3.1 The variable model . . . . . . . . . . . . . . . . . . . . . . . . . . 14

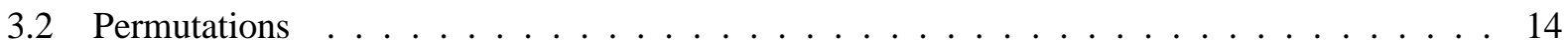

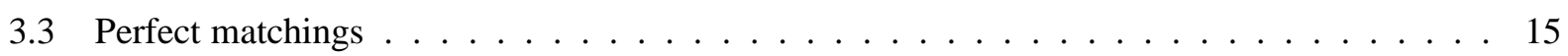

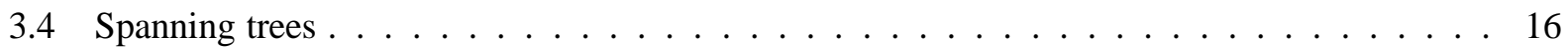

3.5 Composition of resampling oracles for product spaces . . . . . . . . . . . . 18

4 Applications 18

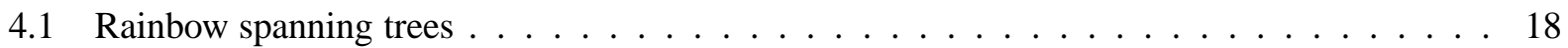

4.2 Rainbow matchings . . . . . . . . . . . . . . . . . . . . . . 20

4.3 Latin transversals . . . . . . . . . . . . . . . . . . . . 22

5 Analysis of the algorithm $\quad 23$

5.1 Stable set sequences and the coupling argument . . . . . . . . . . . . . . 24

5.2 A simple analysis: the General Lovász Lemma criterion, with slack . . . . . . . . . . . 25

5.3 Preliminaries on Shearer's criterion . . . . . . . . . . . . . . . . . 27

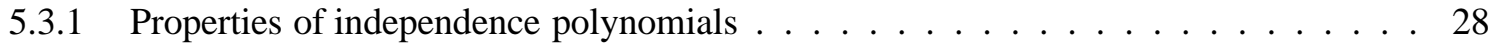

5.3.2 Connection to stable set sequences . . . . . . . . . . . . . . . . . 31

5.4 Shearer's criterion with slack $\ldots \ldots \ldots \ldots \ldots \ldots \ldots \ldots$

5.5 Quantification of slack in Shearer's criterion . . . . . . . . . . . . . . . . 34

5.6 The General LLL criterion, without slack . . . . . . . . . . . . . . . . . . . . . . . . . .

5.7 The cluster expansion criterion . . . . . . . . . . . . . . . . 37

5.7 .1 Proof of Lemma $5.41 \ldots \ldots \ldots \ldots \ldots$

5.7.2 Relationship between cluster expansion and stable set sequences . . . . . . . . . . 40

6 Conclusions 40

A A counterexample to the witness tree lemma 44 


\section{Introduction}

The Lovász Local Lemma (LLL) is a powerful tool with numerous uses in combinatorics and theoretical computer science. If a given probability space and collection of events satisfy a certain condition, then the LLL asserts the existence of an outcome that simultaneously avoids those events. The classical formulation of the LLL [15, 37] is as follows.

Let $\Omega$ be a probability space with probability measure $\mu$. Let $E_{1}, \ldots, E_{n}$ be certain "undesired" events in that space. Let $G$ be an undirected graph with vertex set $[n]=\{1, \ldots, n\}$. The edges of $G$ are denoted $E(G)$. Let $\Gamma(i)=\{j \neq i:\{i, j\} \in E(G)\}$ be the neighbors of vertex $i$. Also, let $\Gamma^{+}(i)=\Gamma(i) \cup\{i\}$ and let $\Gamma^{+}(I)=\bigcup_{i \in I} \Gamma^{+}(i)$ for $I \subseteq[n]$.

Theorem 1.1 (General Lovász Local Lemma [15, 37]). Suppose that the events satisfy the following condition that controls their dependences

$$
\operatorname{Pr}_{\mu}\left[E_{i} \mid \cap_{j \in J} \overline{E_{j}}\right]=\operatorname{Pr}_{\mu}\left[E_{i}\right] \quad \forall i \in[n], J \subseteq[n] \backslash \Gamma^{+}(i)
$$

and the following criterion that controls their probabilities

$$
\exists x_{1}, \ldots, x_{n} \in(0,1) \quad \text { such that } \quad \operatorname{Pr}_{\mu}\left[E_{i}\right] \leq x_{i} \prod_{j \in \Gamma(i)}\left(1-x_{j}\right) \quad \forall i \in[n] .
$$

Then $\operatorname{Pr}_{\mu}\left[\bigcap_{i=1}^{n} \overline{E_{i}}\right]>0$.

An equivalent statement of $(\overline{\mathrm{Dep}})$ is that the event $E_{i}$ must be independent of the joint distribution on the events $\left\{E_{j}: j \notin \Gamma^{+}(i)\right\}$. When (Dep) holds, $G$ is called a dependency graph. The literature contains several dependency conditions generalizing (Dep) and criteria generalizing (GLL) under which the conclusion of the theorem remains true. We will discuss several such generalizations below.

The LLL can also be formulated [6] in terms of a directed dependency graph instead of an undirected graph, but nearly all applications of which we are aware involve an undirected graph. Accordingly, our work focuses primarily on the undirected case, but we will mention below which of our results extend to the directed case.

Algorithms. Algorithms to efficiently find an outcome in $\bigcap_{i=1}^{n} \overline{E_{i}}$ have been the subject of research for several decades. In 2008, a nearly optimal result was obtained by Moser [29] for a canonical application of the LLL, the bounded-degree $k$-SAT problem. Shortly thereafter, Moser and Tardos [30] extended that result to a general scenario called the "variable model" in which $\Omega$ consists of independent variables, each $E_{i}$ depends on a subset of the variables, and events $E_{i}$ and $E_{j}$ are adjacent in $G$ if there is a variable on which they both depend. Clearly the resulting graph is a dependency graph. The Moser-Tardos algorithm is extremely simple: after drawing an initial sample of the variables, it repeatedly checks if any undesired event occurs, then resamples any such event. Resampling an event means that the variables on which it depends receive fresh samples according to $\mu$. Moser and Tardos prove that, if the (GLL) condition is satisfied, this algorithm will produced the desired outcome after at most $\sum_{i=1}^{n} \frac{x_{i}}{1-x_{i}}$ resampling operations, in expectation.

Numerous extensions of the Moser-Tardos algorithm have been proposed. These extensions can handle more general criteria [24, 33, 1, 25], derandomization [13], exponentially many events [20], distributed scenarios [14], etc. However, these results are restricted to the Moser-Tardos variable model and hence cannot be viewed as algorithmic proofs of the LLL in full generality. There are many known scenarios for 
the LLL and its generalizations that fall outside the scope of the variable model [26, 27]. Section 3 discusses several such scenarios, including random permutations, matchings and spanning trees.

Recently two efficient algorithms have been developed that go beyond the variable model. Harris and Srinivasan [21] extend the Moser-Tardos algorithm to a scenario involving random permutations that originates in work of Erdôs and Spencer [16]. Achlioptas and Iliopoulos [2] developed a novel algorithmic "flaw correction" framework which allows one to model various applications of the LLL in a flexible manner. They show how this captures several applications of the LLL outside the variable model, and even some results that might be beyond typical formulations of the LLL. In contrast to the other results mentioned here, their framework does not involve an underlying measure $\mu$ and is not directly tied to the probabilistic setting of the LLL. This has some benefits, but also some restrictions that seem to prevent it from recovering the LLL in full generality, In particular, their publication [2] does not claim a formal connection with Theorem 1.1 Section 1.5 contains further discussion of the related work.

\subsection{Our contributions}

The primary motivating question for this work is whether there is an "algorithmic proof" of the Lovász Local Lemma in general probability spaces. We answer this question in the following sense: We propose an algorithmic framework for the general Lovász Local Lemma, based on a new notion of resampling oracles. In this framework, we present an algorithm that finds a point in $\bigcap_{i=1}^{n} \overline{E_{i}}$ (avoiding all undesired events) efficiently, if given access to three types of subroutines outlined below (the most crucial one being resampling oracles). Whether these subroutines can be implemented efficiently is an instance-dependent issue, and we discuss this further below. However, we show that the existence of such subroutines is guaranteed by the assumptions of the Lovász Local Lemma. In particular, our algorithm provides a new proof of Theorem 1.1 (with no further assumptions), and several generalizations thereof, as described below. Algorithmically, we reduce the problem of finding a point in $\bigcap_{i=1}^{n} \overline{E_{i}}$ to the problem of implementing the three subroutines that we discuss next.

\subsubsection{Algorithmic assumptions}

In order to discuss algorithms for the LLL in full generality, one must assume some form of access to the probability space at hand. It is natural to assume that one can efficiently sample from $\mu$, and efficiently check whether a given event $E_{i}$ occurs. However, even under these assumptions, finding the desired output can be computationally hard. (We show an example demonstrating this in Section 2.2) Therefore, our framework assumes the existence of one more subroutine that can be used by our algorithm. This leads us to the notion of resampling oracles.

Let us introduce some notation. An atomic event $\omega$ in the probability space $\Omega$ will be called a state. We write $\omega \sim \mu$ to denote that a random state $\omega$ is distributed according to $\mu$, and $\left.\omega \sim \mu\right|_{E_{i}}$ to denote that the distribution is $\mu$ conditioned on $E_{i}$. The resampling oracles are defined with respect to a graph $G$ on $[n]$ with neighborhood structure $\Gamma$ (not necessarily satisfying the (Dep) condition).

The three subroutines required by our algorithm are as follows.

- Sampling from $\mu$ : There is a subroutine that provides an independent random state $\omega \sim \mu$.

- Checking events: For each $i \in[n]$, there is a subroutine that determines whether $\omega \in E_{i}$.

- Resampling oracles: For each $i \in[n]$, there is a randomized subroutine $r_{i}: \Omega \rightarrow \Omega$ with the following properties. 
(R1) If $E_{i}$ is an event and $\left.\omega \sim \mu\right|_{E_{i}}$, then $r_{i}(\omega) \sim \mu$. (The oracle $r_{i}$ removes conditioning on $E_{i}$.)

(R2) For any $j \notin \Gamma^{+}(i)$, if $\omega \notin E_{j}$ then also $r_{i}(\omega) \notin E_{j}$. (Resampling an event cannot cause new non-neighbor events to occur.)

When these conditions hold, we say that $r_{i}$ is a resampling oracle for events $E_{1}, \ldots, E_{n}$ and graph $G$.

If efficiency concerns are ignored, the first two subroutines trivially exist. We show that (possibly inefficient) resampling oracles exist if and only if a certain relaxation of (Dep) holds (see Section 1.3$)$.

Main Result. Our main result is that we can find a point in $\bigcap_{i=1}^{n} \overline{E_{i}}$ efficiently, whenever the three subroutines above have efficient implementations.

Theorem (Informal). Consider any probability space, any events $E_{1}, \ldots, E_{n}$, and any undirected graph $G$ on vertex set $[n]$. If (GLL) is satisfied and if the three subroutines described above are available, then our algorithm finds a state in $\bigcap_{i=1}^{n} \overline{E_{i}}$ efficiently in terms of the number of calls to these subroutines.

We make a more precise statement in the following section. We note that this theorem does not assume that (Dep) holds, and the existence of resampling oracles is actually a strictly weaker condition. Thus, our algorithm provides a new proof of Theorem 1.1 (the existential LLL) under its original assumptions.

\subsection{Our algorithm: MaximalSetResample}

A striking aspect of the work of Moser and Tardos [30] is the simplicity and flexibility of their algorithm - in each iteration, any event $E_{i}$ that occurs can be resampled. We propose a different algorithm that is somewhat less flexible, but whose analysis seems to be simpler in our scenario. Roughly speaking, our algorithm proceeds in iterations where in each iteration we resample events that form an independent set in $G$. The independent set is generated by a greedy algorithm that adds a vertex $i$ and resamples $E_{i}$, if $i$ is not adjacent to the previously selected vertices and $E_{i}$ occurs in the current state. This is repeated until no events occur. Pseudocode for this procedure is shown in Algorithm 1. Nearly identical algorithms have been proposed before, particularly parallel algorithms [30, 24], although our interest lies not in the parallel aspects but rather in making the LLL (and its stronger variants) algorithmic in our general setting.

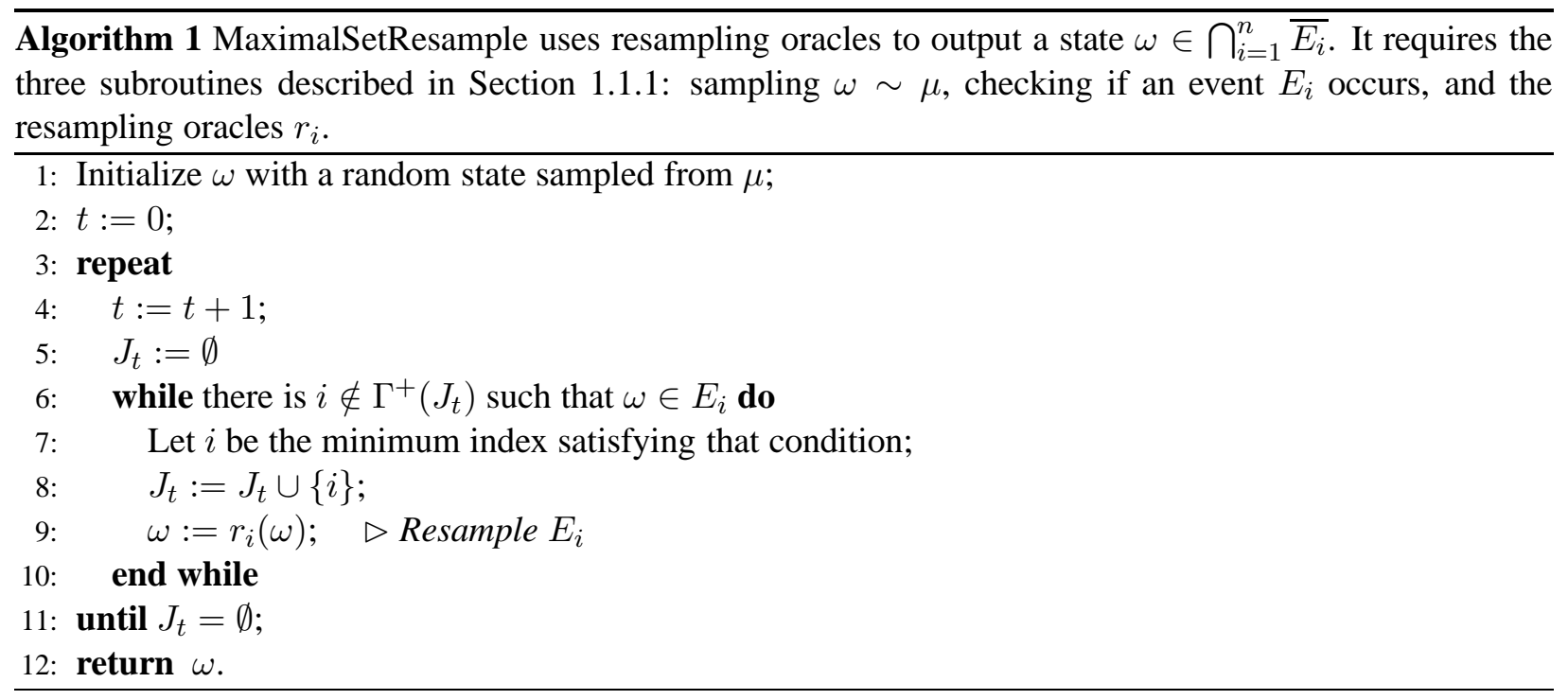


Our algorithmic proof of the LLL amounts to showing that MaximalSetResample terminates, at which point $\omega \in \bigcap_{i=1}^{n} \overline{E_{i}}$ clearly holds. Our bound on the running time of MaximalSetResample is shown by the following theorem, which is proven in Section 5. We note that our bound is at most quadratic in the quantity $\sum_{i=1}^{n} \frac{x_{i}}{1-x_{i}}$ which was the bound proved by Moser and Tardos [30].

Theorem 1.2. Suppose that the events $E_{1}, \ldots, E_{n}$ satisfy (GLL) and that the three subroutines described above in Section 1.1.1 are available. Then the expected number of calls to the resampling oracles before MaximalSetResample terminates is $O\left(\sum_{i=1}^{n} \frac{x_{i}}{1-x_{i}} \sum_{j=1}^{n} \log \frac{1}{1-x_{j}}\right)$.

\subsection{Generalizing the dependency condition}

Erdôs and Spencer [16] showed that Theorem 1.1 still holds when (Dep) is generalized td 1

$$
\operatorname{Pr}_{\mu}\left[E_{i} \mid \cap_{j \in J} \overline{E_{j}}\right] \leq \operatorname{Pr}_{\mu}\left[E_{i}\right] \quad \forall i \in[n], J \subseteq[n] \backslash \Gamma^{+}(i) .
$$

They playfully called this the "lopsidependency" condition, and called $G$ a "lopsidependency graph". This more general condition enables several interesting uses of the LLL in combinatorics and theoretical computer science, e.g., existence of Latin transversals [16] and optimal thresholds for satisfiability [18].

Recall that Theorem 1.2 did not assume $(\overline{D e p})$ and instead assumed the existence of resampling oracles. It is natural to wonder how the latter assumption relates to lopsidependency. We show that the existence of resampling oracles is equivalent to a condition that we call lopsided association, and whose strength lies strictly between (Dep) and (Lop). The lopsided association condition is

$$
\operatorname{Pr}_{\mu}\left[E_{i} \cap F\right] \geq \operatorname{Pr}_{\mu}\left[E_{i}\right] \cdot \operatorname{Pr}_{\mu}[F] \quad \forall i \in[n], \forall F \in \mathcal{F}_{i}
$$

where $\mathcal{F}_{i}$ contains all events $F$ whose indicator variable is a monotone non-decreasing function of the indicator variables of $\left(E_{j}: j \notin \Gamma^{+}(i)\right)$. We call a graph satisfying (LopA a lopsided association graph for events $E_{1}, \ldots, E_{n}$.

Theorem (Informal). Resampling oracles exist for events $E_{1}, \ldots, E_{n}$ and a graph $G$ if and only if $G$ is a lopsided association graph for events $E_{1}, \ldots, E_{n}$.

This equivalence follows essentially from LP duality: The existence of a resampling oracle can be formulated as a transportation problem for which the lopsided association condition is exactly the necessary and sufficient condition for a feasible transportation to exist. Section 2.1 proves this result in detail.

As remarked above, the dependency conditions are related by $(\overline{\text { Dep }}) \Rightarrow(\overline{\text { LopA }}) \Rightarrow($ Lop $)$. The first implication is obvious since (Dep) implies that $E_{i}$ is independent of $F$ in (LopA). To see the second implication, simply take $F=\bigcup_{j \in J} E_{j}$ for any $J \subseteq[n] \backslash \Gamma^{+}(i)$ to obtain that $\operatorname{Pr}_{\mu}\left[E_{i} \mid \cup_{j \in J} E_{j}\right] \geq \operatorname{Pr}_{\mu}\left[E_{i}\right]$. Although lopsided association is formally a stronger assumption than lopsidependency, every use of the LLL with lopsidependency that we have studied actually satisfies the stronger lopsided association condition. We demonstrate this in Section 3 by designing efficient resampling oracles for those scenarios. Consequently, Theorem 1.2 makes the LLL efficient in those scenarios.

As remarked above, Section 2.2 describes a scenario in which (Dep) and (GLL) are satisfied for a dependency graph $G$ but finding a state $\omega \in \bigcap_{i=1}^{n} \overline{E_{i}}$ is computationally hard, assuming standard complexity theoretic beliefs. In that scenario resampling oracles must necessarily exist since $(\overline{D e p})$ is satisfied, but they

\footnotetext{
${ }^{1}$ More precisely, (Lop should be restricted to $J$ for which $\operatorname{Pr}_{\mu}\left[\cap_{j \in J} \overline{E_{j}}\right]>0$. However that restriction is ultimately unnecessary because, in the context of the LLL, the theorem of Erdôs and Spencer implies that $\operatorname{Pr}_{\mu}\left[\cap_{j \in[n]} \overline{E_{j}}\right]>0$.
} 
cannot be efficiently implemented due to the computational hardness. Therefore the equivalence between (LopA and resampling oracles comes with no efficiency guarantees. Nevertheless in all lopsidependency scenarios that we have encountered in applications of the LLL, efficient implementations of the resampling oracles arise naturally from existing work, or can be devised with modest effort. In particular this is the case for random permutations, perfect matchings in complete graphs, and spanning trees in complete graphs, as discussed in Section 3 .

\subsection{Generalizing the LLL criterion}

In the early papers on the LLL [15, 37], the (GLL) criterion relating the dependency graph $G$ and the probabilities $\operatorname{Pr}_{\mu}\left[E_{i}\right]$ was shown to be a sufficient condition to ensure that $\operatorname{Pr}_{\mu}\left[\bigcap_{i=1}^{n} \overline{E_{i}}\right]>0$. Shearer [36] discovered a more general criterion that ensures the same conclusion. In fact, Shearer's criterion is the best possible: whenever his criterion is violated, there exist a corresponding measure $\mu$ and events $E_{1}, \ldots, E_{n}$ for which $\operatorname{Pr}_{\mu}\left[\bigcap_{i=1}^{n} \overline{E_{i}}\right]=0$.

Section 5 formally defines Shearer's criterion and uses it in a fundamental way to prove Theorem 1.2 Moreover, we give an algorithmic proof of the LLL under Shearer's criterion instead of the (GLL) criterion. This algorithm is efficient in typical situations, although the efficiency depends on Shearer's parameters. The following simplified result is stated formally and proven in Section 5.5

Theorem (Informal). Suppose that a graph $G$ and the probabilities $\operatorname{Pr}_{\mu}\left[E_{1}\right], \ldots, \operatorname{Pr}_{\mu}\left[E_{n}\right]$ satisfy Shearer's criterion with $\epsilon$ slack, and that the three subroutines described in Section 1.1.1 are available. Then the expected number of calls to the resampling oracles by MaximalSetResample is $O\left(\frac{n}{\epsilon} \log \frac{1}{\epsilon}\right)$.

We also prove a more refined bound valid for any probabilities satisfying Shearer's criterion. This bound is similar to the bound obtained by Kolipaka and Szegedy [24]; see Section 5.5 for details.

Unfortunately Shearer's criterion is unwieldy and has not seen much use in applications of the LLL. Recently several researchers have proposed criteria of intermediate strength between (GLL) and Shearer's criterion [8, 25]. The first of these, called the cluster expansion criterion, was originally devised by Bissacot et al. [8], and is based on insights from statistical physics. This criterion has given improved results in several applications of the local lemma [9, 21, 31]. Previous algorithmic work has also used the cluster expansion criterion in the variable model [1, 33] and for permutations [21].

We give a new, elementary proof that the cluster expansion criterion implies Shearer's criterion. In contrast, the previous proof is analytic and requires several ideas from statistical physics [8]. As a consequence, we obtain the first purely combinatorial proof that the existential LLL holds under the cluster expansion criterion. Another consequence (Theorem 1.3) is an algorithm for the LLL under the cluster expansion criterion, obtained using our algorithmic results under Shearer's criterion. This generalizes Theorem 1.2 by replacing (GLD) with the cluster expansion criterion, stated below as (CLD). To state the result, we require additional notation: let Ind denote the family of independent sets in the graph $G$.

Theorem 1.3. Suppose that the events $E_{1}, \ldots, E_{n}$ satisfy the following criterion

$$
\exists y_{1}, \ldots, y_{n}>0 \quad \text { such that } \quad \operatorname{Pr}_{\mu}\left[E_{i}\right] \leq \frac{y_{i}}{\sum_{J \subseteq \Gamma^{+}(i), J \in \operatorname{Ind}} \prod_{j \in J} y_{j}} .
$$

and that the three subroutines described in Section 1.1.1 are available. Then the expected number of calls to the resampling oracles before MaximalSetResample terminates is $O\left(\sum_{i=1}^{n} y_{i} \sum_{j=1}^{n} \ln \left(1+y_{j}\right)\right)$. 


\subsection{Techniques and related work}

The breakthrough work of Moser and Tardos [29, 30] stimulated a string of results on algorithms for the LLL. This section reviews the results that are most relevant to our work. Several interesting techniques play a role in the analyses of these previous algorithms. These can be roughly categorized as the entropy method [28, 2], witness trees or witness sequences [30, 21, 24] and forward-looking combinatorial analysis [19].

Moser [29, 28] developed the entropy method to analyze a very simple algorithm for the "symmetric" LLL [15], which incorporates the maximum degree of $G$ and a uniform bound on $\operatorname{Pr}_{\mu}\left[E_{i}\right]$. The entropy method roughly shows that, if the algorithm runs for a long time, a transcript of the algorithm's actions provides a compressed representation of the algorithm's random bits, which is unlikely due to entropy considerations.

Following this, Moser and Tardos [30] showed that a similar algorithm will produce a state in $\bigcap_{i=1}^{n} \overline{E_{i}}$, assuming the independent variable model and the (GLL) criterion. This paper is primarily responsible for the development of witness trees, and proved the "witness tree lemma", which yields an extremely elegant analysis in the variable model. The witness tree lemma has further implications. For example, it allows one to analyze separately for each event its expected number of resamplings. Moser and Tardos also extended the variable model to incorporate a limited form of lopsidependency, and showed that their analysis still holds in that setting.

The main advantage of our result over the Moser-Tardos result is that we address the occurrence of an event through the abstract notion of resampling oracles rather than directly resampling the variables of the variable model. Furthermore we give efficient implementations of resampling oracles for essentially all known probability spaces to which the LLL has been applied. A significant difference with our work is that we do not have an analogue of the witness tree lemma; our approach provides a simpler analysis when the LLL criterion has slack but requires a more complicated analysis to remove the slack assumption. As a consequence, our bound on the number of resampling oracle calls is larger than the Moser-Tardos bound. Our lack of a witness tree lemma is inherent. Appendix A shows that the witness tree lemma is false in the abstract scenario of resampling oracles.

The Moser-Tardos algorithm is known to terminate under criteria more general than (GLL), while still assuming the variable model. Pegden [33] showed that the cluster expansion criterion suffices, whereas Kolipaka and Szegedy [24] showed more generally that Shearer's criterion suffices. We also extend our analysis to the cluster expansion criterion as well as Shearer's criterion, in the more general context of resampling oracles. Our bounds on the number of resampling operations are somewhat weaker than those of [33, 24], but the increase is at most quadratic.

Kolipaka and Szegedy [24] present another algorithm, called GeneralizedResample, whose analysis proves the LLL under Shearer's condition for arbitrary probability spaces. GeneralizedResample is similar to MaximalSetResample in that they both work with abstract distributions and that they repeatedly choose a maximal independent set $J$ of undesired events to resample. However, the way that the bad events are resampled is different: GeneralizedResample needs to sample from $\left.\mu\right|_{\cap_{j \notin \Gamma^{+}(J)}} \overline{E_{j}}$, which is a complicated operation that seems difficult to implement efficiently. Thus MaximalSetResample can be viewed as a variant of GeneralizedResample that can be made efficient in all known scenarios.

Harris and Srinivasan [21] show that the Moser-Tardos algorithm can be adapted to handle certain events in a probability space involving random permutations. Their method for resampling an event is based on the Fischer-Yates shuffle. This scenario can also be handled by our framework; their resampling method perfectly satisfies the criteria of a resampling oracle. The Harris-Srinivasan's result is stronger than ours in that they do prove an analog of the witness tree lemma. Consequently their algorithm requires fewer resamplings than ours, and they are able to derive parallel variants of their algorithm. The work of Harris 
and Srinivasan is technically challenging, and generalizing it to a more abstract setting seems daunting.

Achlioptas and Iliopoulos [2, 3] proposed a general framework for finding "flawless objects", based on actions for addressing flaws. We call this the A-I framework. They show that, under certain conditions, a random walk over such actions rapidly converges to a flawless object. This naturally relates to the LLL by viewing each event $E_{i}$ as a flaw. At the same time, the A-I framework is not tied to the probabilistic formulation of the LLL, and can derive results, such as the greedy algorithm for vertex coloring, that seem to be outside the scope of typical LLL formulations, such as Theorem 1.1 The A-I framework [2, 3] has other restrictions and does not claim to recover any particular form of the LLL. Nevertheless, the framework can accommodate applications of the LLL where lopsidependency plays a role, such as rainbow matchings and rainbow Hamilton cycles. In contrast, our framework embraces the probabilistic formulation and can recover the original existential LLL (Theorem 1.1) in full generality, even incorporating Shearer's generalization. The A-I analysis [2] is inspired by Moser's entropy method. Technically, it entails an encoding of random walks by "witness forests" and combinatorial counting thereof to estimate the length of the random walk. The terminology of witness forests is reminiscent of the witness trees of Moser and Tardos, but conceptually they are different in that the witness forests grow "forward in time" rather than backward. This is conceptually similar to "forward-looking combinatorial analysis", which we discuss next.

Giotis et al. [19] show that a variant of Moser's algorithm gives an algorithmic proof in the variable model of the symmetric LLL. While this result is relatively limited when compared to the results above, their analysis is a clear example of forward-looking combinatorial analysis. Whereas Moser and Tardos use a backward-looking argument to find witness trees in the algorithm's "log", Giotis et al. analyze a forward-looking structure: the tree of resampled events and their dependencies, looking forward in time. This viewpoint seems more natural and suitable for extensions.

Our approach can be roughly described as forward-looking analysis with a careful modification of the Moser-Tardos algorithm, formulated in the framework of resampling oracles. Our main conceptual contribution is the simple definition of the resampling oracles, which allows the resamplings to be readily incorporated into the forward-looking analysis. Our modification of the Moser-Tardos algorithm is designed to combine this analysis with the technology of "stable set sequences" [24], defined in Section 5.1, which allows us to accommodate various LLL criteria, including Shearer's criterion. This plays a fundamental role in the full proof of Theorem 1.2

Our second contribution is a technical idea concerning slack in the LLL criteria. This idea is a perfectly valid statement regarding the existential LLL as well, although we will exploit it algorithmically. One drawback of the forward-looking analysis is that it naturally leads to an exponential bound on the number of resamplings, unless there is some slack in the LLL criterion; this same issue arises in [2, 19]. Our idea eliminates the need for slack in the (GLL) and (CLL) criteria. We prove that, even if (GLL) or (CLL) are tight, we can instead perform our analysis using Shearer's criterion, which is never tight because it defines an open set. For example, consider the familiar case of Theorem 1.1, and suppose that (GLL) holds with equality, i.e., $\operatorname{Pr}_{\mu}\left[E_{i}\right]=x_{i} \prod_{j \in \Gamma(i)}\left(1-x_{j}\right)$ for all $i$. We show that the conclusion of the LLL remains true even if each event $E_{i}$ actually had the larger probability $\operatorname{Pr}_{\mu}\left[E_{i}\right] \cdot\left(1+\left(2 \sum_{i} \frac{x_{i}}{1-x_{i}}\right)^{-1}\right)$. The proof of this fact crucially uses Shearer's criterion and it does not seem to follow from more elementary tools [15, 37].

Follow-up work. Subsequently, Achlioptas and Iliopoulos generalized their framework further to incorporate our notion of resampling oracles [4]. This subsequent work can be viewed as a unification of their framework and ours; it has the benefit of both capturing the framework of resampling oracles and allowing some additional flexibility (in particular, the possibility of regenerating the measure $\mu$ approximately rather than exactly). We remark that this work is still incomparable with ours, primarily due to the facts that our analysis is performed in Shearer's more general setting, and that our algorithm is efficient even when the 
LLL criteria are tight.

Organization. The rest of the paper is organized as follows. In Section 2 , we discuss the connection between resampling oracles and the assumptions of the Lovász Local Lemma. We also show here that resampling oracles as well as the LLL itself can be computationally hard in general. In Section 3, we show concrete examples of efficient implementations of resampling oracles. In Section 4 we discuss several applications of these resampling oracles. Finally, in Section 5 we present the full analysis of our algorithm.

\section{Resampling oracles: existence and efficiency}

The algorithms in this paper make no reference to the lopsidependency condition (Lop) and instead assume the existence of resampling oracles. In Section 2.1 we show that there is a close relationship between these two assumptions: the existence of a resampling oracle for each event is equivalent to the condition (LopA), which is a strengthening of (Lop).

We should emphasize that the efficiency of an implementation of a resampling oracle is a separate issue. There is no general guarantee that resampling oracles can be implemented efficiently. Indeed, as we show in Section 2.2, there are applications of the LLL such that the resampling oracles are hard to implement efficiently, and finding a state avoiding all events is computationally hard, under standard computational complexity assumptions.

Nevertheless, this is not an issue in common applications of the LLL: resampling oracles exist and can be implemented efficiently in all uses of the LLL of which we are aware, even those involving lopsidependency. Section 3 has a detailed discussion of several scenarios.

\subsection{Existence of resampling oracles}

This section proves an equivalence lemma connecting resampling oracles with the notion of lopsided association. First, let us define formally what we call a resampling oracle.

Definition 2.1. Let $E_{1}, \ldots, E_{n}$ be events on a space $\Omega$ with a probability measure $\mu$, and let $G=([n], E)$ be a graph with neighbors of $i \in[n]$ denoted by $\Gamma(i)$. Let $r_{i}$ be a randomized procedure that takes a state $\omega \in \Omega$ and outputs a state $r_{i}(\omega) \in \Omega$. We say that $r_{i}$ is a resampling oracle for $E_{i}$ with respect to $G$, if

(R1) For $\left.\omega \sim \mu\right|_{E_{i}}$, we obtain $r_{i}(\omega) \sim \mu$. (The oracle $r_{i}$ removes conditioning on $E_{i}$.)

(R2) For any $j \notin \Gamma^{+}(i)=\Gamma(i) \cup\{i\}$, if $\omega \notin E_{j}$ then also $r_{i}(\omega) \notin E_{j}$. (Resampling an event cannot cause new non-neighbor events to occur.)

Next, let us define the notion of a lopsided association graph. We denote by $E_{i}[\omega]$ the $\{0,1\}$-valued function indicating whether $E_{i}$ occurs at a state $\omega \in \Omega$.

Definition 2.2. A graph $G$ with neighborhood function $\Gamma$ is a lopsided association graph for events $E_{1}, \ldots, E_{n}$ if

$$
\operatorname{Pr}_{\mu}\left[E_{i} \cap F\right] \geq \operatorname{Pr}_{\mu}\left[E_{i}\right] \cdot \operatorname{Pr}_{\mu}[F] \quad \forall i \in[n], \forall F \in \mathcal{F}_{i}
$$

where $\mathcal{F}_{i}$ contains all events $F$ such that $F[\omega]$ is a monotone non-decreasing function of the functions $\left(E_{j}[\omega]: j \notin \Gamma^{+}(i)\right)$.

Lemma 2.3. Consider a fixed $i \in[n]$ and assume $\operatorname{Pr}_{\mu}\left[E_{i}\right]>0$. The following statements are equivalent. 
(a) There exists a resampling oracle $r_{i}$ satisfying the conditions $(\mathrm{R} 1)$ and $(\mathrm{R} 2)$ with respect to a neighborhood $\Gamma^{+}(i)$ (ignoring issues of computational efficiency).

(b) $\operatorname{Pr}_{\mu}\left[E_{i} \cap F\right] \geq \operatorname{Pr}_{\mu}\left[E_{i}\right] \cdot \operatorname{Pr}_{\mu}[F]$ for any event $F \in \mathcal{F}_{i}$.

Corollary 2.4. Resampling oracles $r_{1}, \ldots, r_{n}$ exist for events $E_{1}, \ldots, E_{n}$ with respect to a graph $G$ if and only if $G$ is a lopsided association graph for $E_{1}, \ldots, E_{n}$. Both statements imply that the lopsidependency condition (Lop) holds.

Proof (of Lemma2.3). (a) $\Rightarrow$ (b): Consider the coupled states $\left(\omega, \omega^{\prime}\right)$ where $\left.\omega \sim \mu\right|_{E_{i}}$ and $\omega^{\prime}=r_{i}(\omega)$. By (R1), $\omega^{\prime} \sim \mu$. For any event $F \in \mathcal{F}_{i}$, if $F$ does not occur at $\omega$ then it does not occur at $\omega^{\prime}$ either, due to (R2). This establishes that

$$
\operatorname{Pr}_{\mu}[F]=\mathbf{E}_{\omega^{\prime} \sim \mu}\left[F\left[\omega^{\prime}\right]\right] \leq \mathbf{E}_{\omega \sim \mu \mid E_{i}}[F[\omega]]=\operatorname{Pr}_{\mu}\left[F \mid E_{i}\right],
$$

which implies $\operatorname{Pr}_{\mu}\left[F \cap E_{i}\right] \geq \operatorname{Pr}_{\mu}[F] \cdot \operatorname{Pr}_{\mu}\left[E_{i}\right]$. In particular this implies $\left[\overline{\text { Lop }}\right.$, by taking $F=\bigcup_{j \in J} E_{j}$.

(b) $\Rightarrow$ (a): We begin by formulating the existence of a resampling oracle as the following transportation problem. Consider a bipartite graph $(U \cup W, E)$, where $U$ and $W$ are disjoint, $U$ represents all the states $\omega \in \Omega$ satisfying $E_{i}$, and $W$ represents all the states $\omega \in \Omega$. Edges represent the possible actions of the resampling oracle: $(u, w) \in E$ if $u$ satisfies every event among $\left(E_{j}: j \notin \Gamma^{+}(i)\right)$ that $w$ satisfies. Each vertex has an associated weight: For $w \in W$, we define $p_{w}=\operatorname{Pr}_{\mu}[w]$, and for $u \in U, p_{u}=$ $\operatorname{Pr}_{\mu}[u] / \operatorname{Pr}_{\mu}\left[E_{i}\right]$, i.e, $p_{u}$ is the probability of $u$ conditioned on $E_{i}$. We claim that the resampling oracle $r_{i}$ exists if and only if there is an assignment $f_{u w}$ of values to the edges such that

$$
\begin{array}{ll}
\sum_{w:(u, w) \in E} f_{u w}=p_{u} & \forall u \in U \\
\sum_{u:(u, w) \in E} f_{u w}=p_{w} & \forall w \in W \\
f_{u w} \geq 0 & \forall u \in U, w \in W .
\end{array}
$$

Such an assignment is called a feasible transportation. Given such a transportation, the resampling oracle is defined naturally by following each edge from $u \in U$ with probability $f_{u w} / p_{u}$, and the resulting distribution on $W$ is $p_{w}$. Conversely, for a resampling oracle which, for a given state $u \in U$, generates $w \in W$ with probability $q_{u w}$, we define $f_{u w}=p_{u} q_{u w}$. This assignment satisfies (1).

Our goal at this point is show that (b) implies feasibility of (1). A condition that is equivalent to (1), but more convenient for our purposes, can be determined from LP duality [34, Theorem 21.11]. A feasible transportation exists if and only if

$$
\begin{array}{ll}
\text { (2.1) } & \sum_{u \in U} p_{u}=\sum_{w \in W} p_{w} \\
\text { (2.2) } & \sum_{u \in A} p_{u} \leq \sum_{w \in \Gamma(A)} p_{w} \quad \forall A \subseteq U,
\end{array}
$$

where $\Gamma(A)=\{w \in W: \exists u \in A$ s.t. $(u, w) \in E\}$. This is an extension of Hall's condition for the existence of a perfect matching.

Our goal at this point is show that (b) implies feasibility of (2). Let us now simplify (2). Fix any $A \subseteq$ $U$. The neighborhood $\Gamma(A)$ consists of states satisfying at most those events among $\left\{E_{j}: j \notin \Gamma^{+}(i)\right\}$ satisfied by some state in $A$. Thus $\Gamma(A)$ corresponds to an event $F^{\prime}$ such that $F^{\prime}[\omega]$ is a non-increasing function of $\left(E_{j}[\omega]: j \notin \Gamma^{+}(i)\right)$. Next observe that, if the set of events among $\left\{E_{j}: j \in \Gamma^{+}(i)\right\}$ satisfied by $u^{\prime} \in U$ is a subset of those satisfied by $u \in U$, then $\Gamma\left(u^{\prime}\right) \subseteq \Gamma(u)$. Suppose that, for each $u \in A$, we add to $A$ all such vertices $u^{\prime}$. Doing so can only increase the left-hand side of (2) 2), but does not increase the right-hand side as $\Gamma(A)$ remains unchanged (since $\Gamma\left(u^{\prime}\right) \subseteq \Gamma(u)$ ). Furthermore, the resulting set $A$ 
corresponds to the same event $F^{\prime}$, but restricted to the states in $U$. Let us call such a set $A$ non-increasing. Let $\left(2^{*}\right)$ denote the simplification of (2) in which we restrict to non-increasing $A$. We have argued that (2) and $\left(2^{*}\right)$ are equivalent.

Our goal at this point is show that (b) implies feasibility of $\left(2^{*}\right)$. One may easily see that (b) is equivalent to

$$
\operatorname{Pr}_{\mu}\left[\bar{F} \cap E_{i}\right] \leq \operatorname{Pr}_{\mu}[\bar{F}] \cdot \operatorname{Pr}_{\mu}\left[E_{i}\right] \quad \forall F \in \mathcal{F}_{i} .
$$

Assuming $\operatorname{Pr}\left[E_{i}\right]>0$, we can rewrite this as $\operatorname{Pr}_{\mu}\left[\bar{F} \mid E_{i}\right] \leq \operatorname{Pr}_{\mu}[\bar{F}] \forall F \in \mathcal{F}_{i}$. Now consider using this inequality with $F=\overline{F^{\prime}}$ for each $F^{\prime}$ corresponding to some non-increasing set $A \subseteq U$. We then have $\operatorname{Pr}_{\mu}\left[F^{\prime} \mid E_{i}\right]=\sum_{u \in A} p_{u}$ and $\operatorname{Pr}_{\mu}\left[F^{\prime}\right]=\sum_{w \in \Gamma(A)} p_{w}$. This verifies the feasibility of $\left(2{ }^{k}\right)$.

\subsubsection{Example: monotone events on lattices}

This section presents an example of a setting where Lemma 2.3 implies the existence of a non-trivial resampling oracle, even though the lopsided association graph is empty. This setting was previously known to have connections to the existential LLL [26]. The probability space here is $\Omega=\{0,1\}^{M}$, viewed in the natural way as the Boolean lattice with operations $\wedge$ (meet) and $\vee$ (join), and with the partial order denoted $\geq$. Let $\mu:\{0,1\}^{M} \rightarrow[0,1]$ be a probability distribution, i.e., $\sum_{x \in\{0,1\}^{M}} \mu(x)=1$. We assume that $\mu$ is log-supermodular, meaning that

$$
\mu(x \vee y) \mu(x \wedge y) \geq \mu(x) \mu(y) \quad \forall x, y \in\{0,1\}^{M} .
$$

As an example, any product distribution is log-supermodular. Consider monotone increasing events $E_{i}$, i.e., such that $x^{\prime} \geq x \in E_{i} \Rightarrow x^{\prime} \in E_{i}$. Note that any monotone increasing function of such events is again monotone increasing. It follows directly from the FKG inequality [6] that condition (b) of Lemma 2.3 is satisfied for such events with an empty lopsided association graph. Therefore, a resampling oracle exists in this setting. However, the explicit description of its operation might be complicated and we do not know whether it can be implemented efficiently in general.

Alternatively, the existence of the resampling oracle can be proved directly, using a theorem of Holley [22, Theorem 6]. The resampling oracle is described in Algorithm 2 , The reader can verify that this satisfies the assumptions (R1) and (R2), using Holley's Theorem.

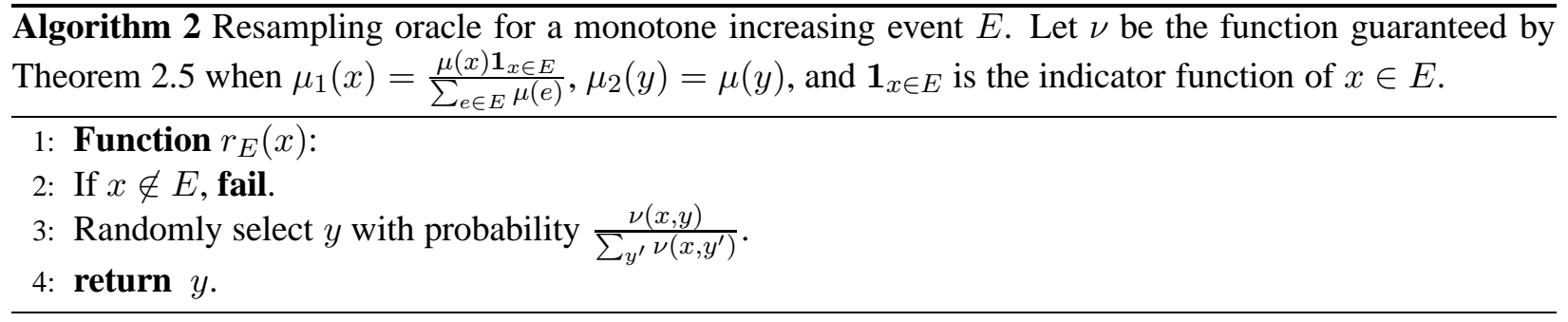

Theorem 2.5 (Holley's Theorem). Let $\mu_{1}$ and $\mu_{2}$ be probability measures on $\{0,1\}^{M}$ satisfying

$$
\mu_{1}(x \vee y) \mu_{2}(x \wedge y) \geq \mu_{1}(x) \mu_{2}(y) \quad \forall x, y \in\{0,1\}^{M} .
$$


Then there exists a probability distribution $\nu:\{0,1\}^{M} \times\{0,1\}^{M} \rightarrow \mathbb{R}$ satisfying

$$
\begin{gathered}
\mu_{1}(x)=\sum_{y} \nu(x, y) \\
\mu_{2}(y)=\sum_{x} \nu(x, y) \\
\nu(x, y)=0 \text { unless } x \geq y .
\end{gathered}
$$

\subsection{Computational hardness of the LLL}

This section considers whether the LLL can always be made algorithmic. We show that, even in fairly simple scenarios where the LLL applies, finding the desired output can be computationally hard, a fact that surprisingly seems to have been overlooked. We first observe that the question of algorithmic efficiency must be stated carefully otherwise hardness is trivial.

A trivial example. Given a Boolean formula $\phi$, let the probability space be $\Omega=\{0,1\}$, and let $\mu$ be the uniform measure on $\Omega$. There is a single event $E_{1}$ defined to be $E_{1}=\{1\}$ if $\phi$ is satisfiable, and $E_{1}=\{0\}$ if $\phi$ is not satisfiable. Since $\operatorname{Pr}\left[E_{1}\right]=1 / 2$, the (GLL) criterion holds trivially with $x_{1}=1 / 2$. The LLL gives the obvious conclusion that there is a state $\omega \notin E$. Yet, finding this state requires deciding satisfiability of $\phi$, which is NP-complete.

The reason that this example is trivial is that even deciding whether the undesired event has occurred is computationally hard. A more meaningful discussion of LLL efficiency ought to rule out this trivial example by considering only scenarios that satisfy some reasonable assumptions. With that in mind, we will assume that

- there is a probability space $\Omega$, whose states can be described by $m$ bits;

- a graph $G$ satisfying $(\overline{D e p})$ for events $E_{1}, \ldots, E_{n}$ is explicitly provided;

- $x_{1}, \ldots, x_{n} \in(0,1)$ satisfying the (GLL) conditions are provided, and $\sum_{i=1}^{n} \frac{x_{i}}{1-x_{i}}$ is at most poly $(n)$;

- there is a subroutine that provides an independent random state $\omega \sim \mu$ in poly $(m)$ time;

- for each $i \in[n]$, there is a subroutine which determines for any given $\omega \in \Omega$ whether $\omega \in E_{i}$, in poly $(m)$ time.

As far as we know, no prior work refutes the possibility that there is an algorithmic form of the LLL, with running time poly $(m, n)$, in this general scenario.

Our results imply that resampling oracles do exist in this general scenario, so it is only the question of whether these resampling oracles are efficient that prevents Theorem 1.2 from providing an efficient algorithm. Nevertheless, we show that there is an instance of the LLL that satisfies the reasonable assumptions stated above, but for which finding a state in $\bigcap_{i} \overline{E_{i}}$ requires solving a problem that is computationally hard (under standard computational complexity assumptions). As a consequence, we conclude that the resampling oracles cannot always be implemented efficiently, even under the reasonable assumptions of this general scenario.

We remark that NP-completeness is not the right notion of hardness here [32]. Problems in NP involve deciding whether a solution exists, whereas the LLL guarantees that a solution exists, and the goal is to explicitly find a solution. Our result is instead based on hardness of the discrete logarithm problem, a standard belief in computational complexity theory. In the following, $\operatorname{GF}\left(p^{n}\right)$ for a prime $p$ and integer $n$ denotes a finite field of order $p^{n}$, and $\mathrm{GF}^{*}\left(p^{n}\right)$ its multiplicative group of nonzero elements.

Theorem 2.6. There are instances of events $E_{1}, \ldots, E_{n}$ on a probability space $\Omega=\{0,1\}^{n}$ under the uniform probability measure, such that

- the events $E_{i}$ are mutually independent; 
- for each $i \in[n]$, the condition $\omega \in E_{i}$ can be checked in poly $(n)$ time for given $\omega \in \Omega$;

- the (GLL) conditions are satisfied with $x_{i}=1 / 2$ for each $i \in[n]$;

but finding a state in $\bigcap_{i=1}^{n} \overline{E_{i}}$ is as hard as solving the discrete logarithm problem in $\operatorname{GF}^{*}\left(2^{n}\right)$.

Remark. Superficially, this result seems to contradict the fact that the LLL can be made algorithmic in the variable model [30], where events are defined on underlying independent random variables. The key point is that the variable model also relies on a particular type of dependency graph (defined by shared variables) which might be more conservative than the true dependencies between the events. Theorem 2.6 shows that, even if the probability space consists of independent $\{0,1\}$ random variables, the LLL cannot in general be made algorithmic if the true dependencies are considered.

Proof. Consider an instance of the discrete logarithm problem in the multiplicative group $\mathrm{GF}^{*}\left(2^{n}\right)$. The input is a generator $g$ of $\operatorname{GF}^{*}\left(2^{n}\right)$ and an element $h \in \mathrm{GF}^{*}\left(2^{n}\right)$. The goal is to find an integer $1 \leq k \leq 2^{n}-1$ such that $g^{k}=h$. We define an instance of $n$ events on $\Omega=\{0,1\}^{n}$ as follows.

We identify $\Omega=\{0,1\}^{n}$ with $\left[2^{n}\right]$ as well as $\operatorname{GF}\left(2^{n}\right)$ in a natural way. We define $f:\left[2^{n}\right] \rightarrow \operatorname{GF}\left(2^{n}\right)$ by $f(0)=0$ and $f(x)=g^{x}$ for $x \neq 0$, where the exponentiation is performed in $\operatorname{GF}\left(2^{n}\right)$. For each $i \in[n]$, we define an event $E_{i}$ that occurs for $\omega \in\{0,1\}^{n}$ iff $(f(\omega))_{i}=1-h_{i}$. This is a condition that can be checked in time poly $(n)$, by computing $f(\omega)=g^{\omega}$ where we interpret $\omega$ as $\sum_{i=0}^{n-1} \omega_{i} 2^{i}$ and compute $g^{\omega}$ by taking squares iteratively.

Observe that for $\omega$ distributed uniformly in $\Omega=\{0,1\}^{n}, f(\omega)$ is again distributed uniformly in $\Omega$, since $f$ is a bijection ( 0 is mapped to 0 , and $f(\omega)$ for $\omega \neq 0$ generates each element of the multiplicative group $\mathrm{GF}^{*}\left(2^{n}\right)$ exactly once). Therefore, the probability of $E_{i}$ is $1 / 2$, for each $i \in[n]$. Further, the events $E_{1}, \ldots, E_{n}$ are mutually independent, since for any $J \subseteq[n], \bigcap_{j \in J} E_{j} \cap \bigcap_{j^{\prime} \notin J} \overline{E_{j^{\prime}}}$ occurs iff $f(\omega)=h \oplus \mathbf{1}_{J}$, which happens with probability $1 / 2^{n}$. Here $\mathbf{1}_{J} \in\{0,1\}^{n}$ is the indicator vector for the set $J$, and $\oplus$ denotes addition in $\operatorname{GF}\left(2^{n}\right)$ (i.e., component-wise xor in $\{0,1\}^{n}$ ). Hence the dependency graph is empty, and the LLL with parameters $x_{i}=1 / 2$ trivially implies that there exists a state $\omega$ avoiding all the events. In this instance, we know explicitly that the state avoiding all the events is $f^{-1}(h)$. Therefore, if we had an efficient algorithm to find this point for any given $h \in \mathrm{GF}^{*}\left(2^{n}\right)$, we would also have an efficient algorithm for the discrete logarithm problem in $\mathrm{GF}\left(2^{n}\right)$.

\section{Implementation of resampling in specific settings}

In this section, we present efficient implementations of resampling oracles in four application settings: independent random variables (which was the setting of [30]), random permutations (handled by [21]), perfect matchings in complete graphs (some of whose applications are made algorithmic by [2]), and spanning trees in complete graphs (which is a new scenario that we can handle). To be more precise, resampling oracles also depend on the types of events and dependencies that we want to handle 2 In the setting of independent random variables, we can handle arbitrary events with dependencies defined by overlapping relevant variables, just like [30]. In the setting of permutations, we handle the appearance of patterns in permutations as in [21]. In the settings of matchings and spanning trees, we consider the "canonical events" defined by [26], characterized by the appearance of a certain subset of edges. We also show in Section 3.5 how resampling oracles for a certain probability space can be extended in a natural way to products of such probability spaces (for example, how to go from resampling oracles for one random permutation to a collection of independent random permutations). These settings cover all the applications of the lopsided LLL that we are aware of.

\footnotetext{
${ }^{2}$ In Section 2.2 we give an example of events on independent random variables for which resampling oracles exist but cannot be made efficient.
} 


\subsection{The variable model}

This is the most common setting, considered originally by Moser and Tardos [30]. Here, $\Omega$ has a product structure corresponding to independent random variables $\left\{X_{a}: a \in \mathcal{U}\right\}$. The probability measure $\mu$ here is a product measure. Each bad event $E_{i}$ depends on a particular subset of variables $A_{i}$, and two events are independent iff $A_{i} \cap A_{j}=\emptyset$.

Here our algorithmic assumptions correspond exactly to the Moser-Tardos framework [30]. Sampling from $\mu$ means generating a fresh set of random variables independently. The resampling oracle $r_{i}$ takes a state $\omega$ and replaces the random variables $\left\{X_{a}: a \in A_{i}\right\}$ by fresh random samples. It is easy to see that the assumptions are satisfied: in particular, a random state sampled from $\mu$ conditioned on $E_{i}$ has all variables outside of $A_{i}$ independently random. Hence, resampling the variables of $A_{i}$ produces the distribution $\mu$. Clearly, resampling $\left\{X_{a}: a \in A_{i}\right\}$ does not affect any events whose variables do not intersect $A_{i}$.

We note that this resampling oracle is also consistent with the notion of lopsidependency on product spaces considered by [30]: They call two events $E_{i}, E_{j}$ lopsidependent, if $A_{i} \cap A_{j} \neq \emptyset$ and it is possible to cause $E_{j}$ to occur by resampling $A_{i}$ in a state where $E_{i}$ holds but $E_{j}$ does not (the definition in [30] is worded differently but equivalent to this). This is exactly the condition that we require our resampling oracle to satisfy.

\subsection{Permutations}

The probability space $\Omega$ here is the space of all permutations $\pi$ on a set $[n]$, with a uniform measure $\mu$. The bad events are assumed to be "simple" in the following sense: Each bad event $E_{i}$ is defined by a "pattern" $P\left(E_{i}\right)=\left\{\left(x_{1}, y_{1}\right), \ldots,\left(x_{t(i)}, y_{t(i)}\right)\right\}$. The event $E_{i}$ occurs if $\pi\left(x_{j}\right)=y_{j}$ for each $1 \leq j \leq t(i)$. Let $\operatorname{vbl}\left(E_{i}\right)=\left\{x: \exists y,(x, y) \in P\left(E_{i}\right)\right\}$ denote the variables of $\pi$ relevant to event $E_{j}$. Let us define a relation $i \sim i^{\prime}$ to hold iff there are pairs $(x, y) \in P\left(E_{i}\right),\left(x^{\prime}, y^{\prime}\right) \in P\left(E_{i^{\prime}}\right)$ such that $x=x^{\prime}$ or $y=y^{\prime}$; i.e., the two events entail the same value in either the range or domain. This relation defines a lopsidependency graph. It is known that the lopsided LLL holds in this setting.

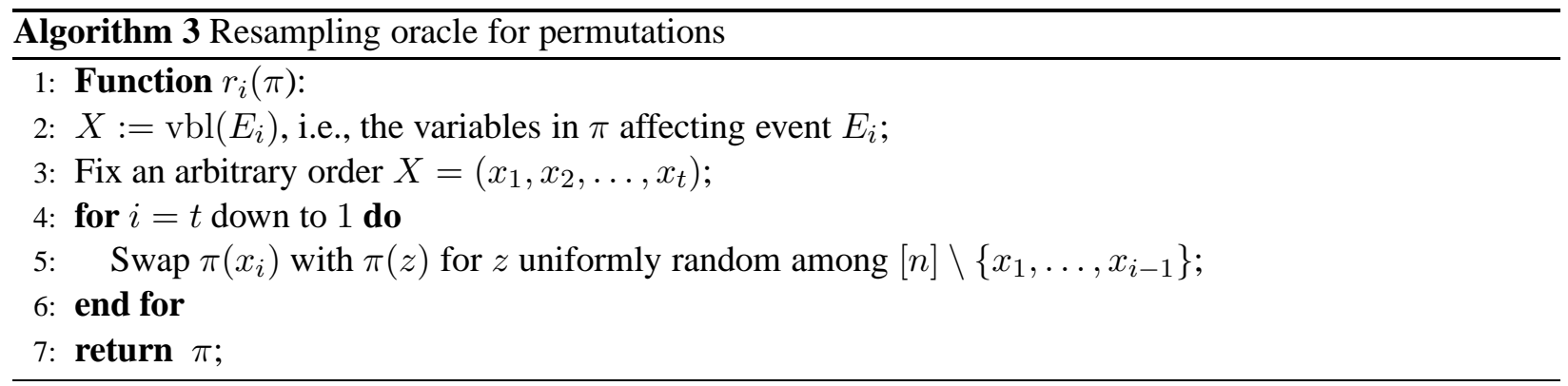

Harris and Srinivasan [21] showed how, under the LLL criteria, a permutation avoiding all bad events can be found algorithmically. We implement the resampling oracle based on their algorithm (see Algorithm 3 ). To prove the correctness of this resampling oracle within our framework, we need the following lemma.

Lemma 3.1. Suppose that a permutation $\pi$ has some arbitrary fixed assignment on the variables in $X$, $\left.\pi\right|_{X}=\phi$, and it is uniformly random among all permutations satisfying $\left.\pi\right|_{X}=\phi$. Then the output of Shuffle $(\pi, X)$ is a uniformly random permutation.

The procedure is known as the Fisher-Yates shuffle for generating uniformly random permutations (and was used in [21] as well). In contrast to the full shuffle, we assume that some part of the permutation has 
been shuffled already: $X$ is the remaining portion that still remains to be shuffled, and conditioned on its assignment the rest is uniformly random. This would be exactly the distribution achieved after performing the Fisher-Yates shuffle on the complement of $X$. Our procedure performs the rest of the Fisher-Yates shuffle, which produces a uniformly random permutation. For completeness we give a self-contained proof.

Proof. Let $X=\left\{x_{1}, \ldots, x_{t}\right\}$. By induction, after performing the swap for $x_{i}$, the permutation is uniform among all permutations with a fixed assignment of $\left\{x_{1}, \ldots, x_{i-1}\right\}$ (consistent with $\phi$ ). This holds because, before the swap, the permutation was by induction uniform conditioned on the assignment of $\left\{x_{1}, \ldots, x_{i}\right\}$ being consistent with $\phi$, and we choose a uniformly random swap for $x_{i}$ among the available choices. This makes every permutation consistent with $\phi$ on $\left\{x_{1}, \ldots, x_{i-1}\right\}$ equally likely after this swap.

This verifies the first condition for our resampling oracle. The second condition is that resampling of occurring events does not affect non-neighbor events. This is true because of the following lemma.

Lemma 3.2. The resampling oracle $r_{i}(\pi)$ applied to a permutation satisfying $E_{i}$ does not cause any new event outside of $\Gamma^{+}(I)$ to occur.

Proof. Suppose $E_{j}$ changed its status during a call to $r_{i}(\pi)$. This means that something changed among its relevant variables $\operatorname{vbl}\left(E_{j}\right)$. This could happen in two ways:

(1) either a variable $z \in \operatorname{vbl}\left(E_{j}\right)$ was swapped because $z \in X=\operatorname{vbl}\left(E_{i}\right)$; then clearly $j \in \Gamma^{+}(i)$.

(2) or, a variable in $\operatorname{vbl}\left(E_{j}\right)$, although outside of $X$, received a new value by a swap with some variable in $X=\operatorname{vbl}\left(E_{i}\right)$. Note that in the Shuffle procedure, every time a variable $z$ outside of $X$ changes its value, it is by a swap with a fresh variable of $X$, i.e. one that had not been processed before. Therefore, the value that $z$ receives is one that previously caused $E_{i}$ to occur. If it causes $E_{j}$ to occur, it means that $E_{i}$ and $E_{j}$ share a value in the range space and we have $j \in \Gamma^{+}(i)$ as well.

\subsection{Perfect matchings}

Here, the probability space $\Omega$ is the set of all perfect matchings in $K_{2 n}$, with the uniform measure. This is a setting considered by [2] and it is also related to the setting of permutations. (Permutations on $[n]$ can be viewed as perfect matchings in $K_{n, n}$.) A state here is a perfect matching in $K_{2 n}$, which we denote by $M \in \Omega$. We consider bad events of the following form: $E_{A}$ for a set of edges $A$ occurs if $A \subseteq M$. Obviously, $\operatorname{Pr}_{\mu}\left[E_{A}\right]>0$ only if $A$ is a (partial) matching. Let us define $A \sim B$ iff $A \cup B$ is not a matching. It was proved in [26] that this defines a lopsidependency graph.

Our goal is to implement a resampling oracle in this setting. We describe such an operation in Algorithm 4

Lemma 3.3. Let $A$ be a matching in $K_{2 n}$ and let $M$ be distributed uniformly among perfect matchings in $K_{2 n}$ such that $A \subseteq M$. Then after calling the resampling oracle, $r_{A}(M)$ is a uniformly random perfect matching.

Proof. We prove by induction that at any point, $M^{\prime}$ is a uniformly random perfect matching conditioned on containing $A^{\prime}$. This is satisfied at the beginning: $M^{\prime}=M, A^{\prime}=A$ and $M$ is uniformly random conditioned on $A \subseteq M$.

Assume this is true at some point, we pick $(u, v) \in A^{\prime}$ arbitrarily and $(x, y) \in M^{\prime} \backslash A^{\prime}$ uniformly at random. Denote the vertices covered by $M^{\prime} \backslash A^{\prime}$ by $V\left(M^{\prime} \backslash A^{\prime}\right)$. Observe that for a uniformly random perfect matching on $V\left(M^{\prime} \backslash A^{\prime}\right) \cup\{u, v\}$, the edge $(u, v)$ should appear with probability $1 /\left(2\left|M^{\prime} \backslash A^{\prime}\right|+1\right)$ since $u$ has $2\left|M^{\prime} \backslash A^{\prime}\right|+1$ choices to be matched with and $v$ is 1 of them. Consequently, we keep the edge $(u, v)$ 


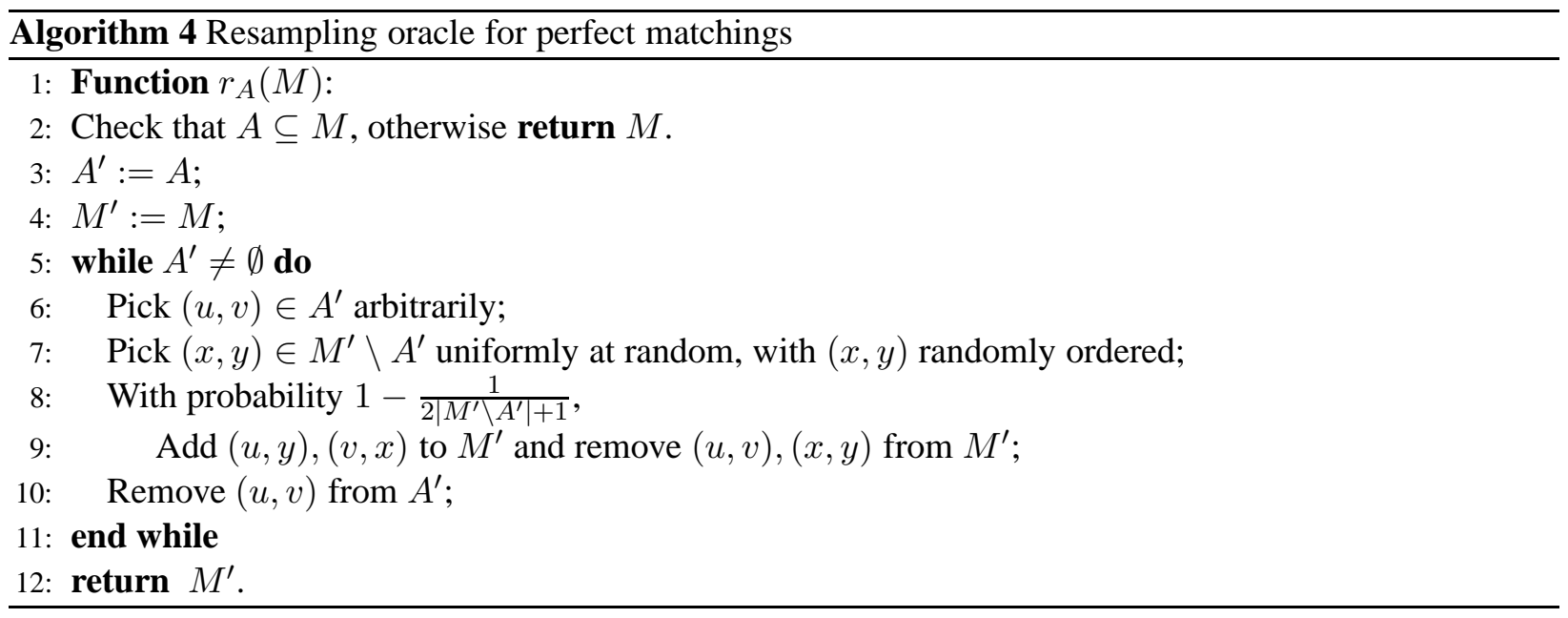

with probability $1 /\left(2\left|M^{\prime} \backslash A^{\prime}\right|+1\right)$ and conditioned on this $M^{\prime} \backslash A^{\prime}$ is uniformly random by the inductive hypothesis. Conditioned on $(u, v)$ not being part of the matching, we re-match $(u, v)$ with another random edge $(x, y) \in M^{\prime} \backslash A^{\prime}$ where $(x, y)$ is randomly ordered. In this case, $u$ and $v$ get matched to a uniformly random pair of vertices $x, y \in V\left(M^{\prime} \backslash A^{\prime}\right)$, as they should be. The rest of the matching $M^{\prime} \backslash A^{\prime} \backslash\{(x, y)\}$ is uniformly random on $V\left(M^{\prime} \backslash A^{\prime} \backslash\{x, y\}\right)$ by the inductive hypothesis.

Therefore, after each step $M^{\prime} \backslash A^{\prime}$ is uniformly random conditioned on containing $A^{\prime}$. At the end, $A^{\prime}=\emptyset$ and $M^{\prime}$ is uniformly random.

Lemma 3.4. The resampling oracle $r_{A}(M)$ applied to a perfect matching satisfying event $E_{A}$ does not cause any new event $E_{B}$ such that $B \notin \Gamma^{+}(A)$.

Proof. Observe that all the new edges that the resampling oracle adds to $M$ are incident to some vertex matched by $A$. So if an event $E_{B}$ was not satisfied before the operation and it is satisfied afterwards, it must be the case that $B$ contains some edge not present in $A$ but sharing a vertex with $A$. Hence, $A \cup B$ is not a matching and $A \sim B$.

\subsection{Spanning trees}

Here, the probability space $\Omega$ is the set of all spanning trees in $K_{n}$. Let us consider events $E_{A}$ for a set of edges $A$, where $E_{A}$ occurs for $T \in \Omega$ iff $A \subseteq T$. Define $A \sim B$ for distinct $A, B$ unless $A$ and $B$ are vertex-disjoint. Lu et al. [26, Lemma 7] show that this in fact defines a dependency graph for spanning trees. It is worth emphasizing that in this scenario the $(\overline{D e p})$ condition holds (the more general condition $(\overline{L o p})$ is not needed), but the scenario does not fall within the scope of the Moser-Tardos variable model. It does fall within the scope of our framework, but one must design a non-trivial resampling oracle.

To implement a resampling oracle in this setting, we will use as a subroutine an algorithm to generate a uniformly random spanning tree in a given graph $G$. This can be done efficiently by several methods, for example by a random walk [10].

Lemma 3.5. If $A$ is a fixed forest and $T$ is a uniformly random spanning tree in $K_{n}$ conditioned on $A \subseteq T$, then $r_{A}(T)$ produces a uniformly random spanning tree in $K_{n}$. 


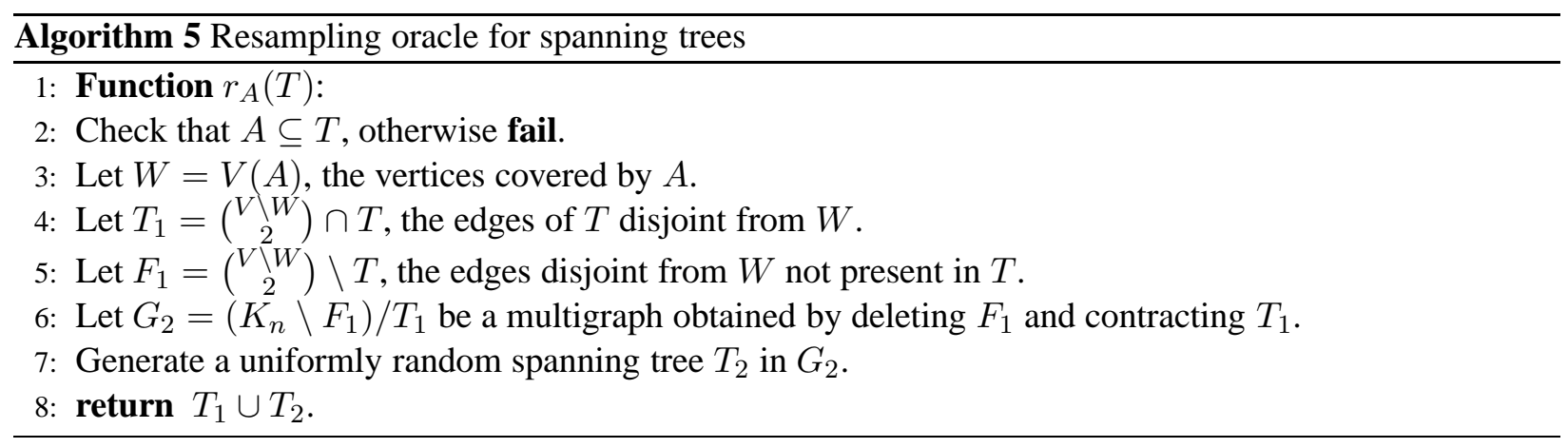

Proof. First, observe that since $T_{2}$ is a spanning tree of $G_{2}=\left(K_{n} \backslash F_{1}\right) / T_{1}$, it is also a spanning tree of $K_{n} / T_{1}$ where $T_{1}$ is a forest, and therefore $T_{1} \cup T_{2}$ is a spanning tree of $K_{n}$. We need to prove that it is a uniformly random spanning tree.

First, we appeal to a known result [26, Lemma 6] stating that given a forest $F$ in $K_{n}$ with components of sizes (number of vertices) $f_{1}, f_{2}, \ldots, f_{m}$, the number of spanning trees containing $F$ is exactly

$$
n^{n-2} \prod_{i=1}^{m} \frac{f_{i}}{n^{f_{i}-1}} .
$$

Equivalently (since $n^{n-2}$ is the total number of spanning trees), for a uniformly random spanning tree $T$, $\operatorname{Pr}[F \subseteq T]=\prod_{i=1}^{m} f_{i} / n^{f_{i}-1}$. This has the surprising consequence that for vertex-disjoint forests $F_{1}, F_{2}$, we have $\operatorname{Pr}\left[F_{1} \cup F_{2} \subseteq T\right]=\operatorname{Pr}\left[F_{1} \subseteq T\right] \cdot \operatorname{Pr}\left[F_{2} \subseteq T\right]$, i.e., the containment of $F_{1}$ and $F_{2}$ are independent events. (In a general graph, the appearances of different edges in a random spanning tree are negatively correlated, but here we are in a complete graph.)

Let $W=V(A)$ and let $B$ be any forest on $V \backslash W$, i.e., vertex-disjoint from $A$. By the above, the appearance of $B$ in a uniformly random spanning tree is independent of the appearance of $A$. Hence, if $T$ is uniformly random, we have $\operatorname{Pr}[B \subseteq T \mid A \subseteq T]=\operatorname{Pr}[B \subseteq T]$. This implies that the distribution of $T \cap\left(\begin{array}{c}V \backslash W \\ 2\end{array}\right)$ is exactly the same for a uniformly random spanning tree $T$ as it is for one conditioned on $A \subseteq T$ (formally, by applying the inclusion-exclusion formula). Therefore, the forest $T_{1}=T \cap\left(\begin{array}{c}V \backslash W \\ 2\end{array}\right)$ is distributed as it should be in a random spanning tree restricted to $V \backslash W$.

The final step is that we extend $T_{1}$ to a spanning tree $T_{1} \cup T_{2}$, where $T_{2}$ is a uniform spanning tree in $G_{2}=\left(K_{n} \backslash F_{1}\right) / T_{1}$. Note that $G_{2}$ is a multigraph, i.e., it is important that we preserve the multiplicity of edges after contraction. The spanning trees $T_{2}$ in $G_{2}=\left(K_{n} \backslash F_{1}\right) / T_{1}$ are in a one-to-one correspondence with spanning trees in $K_{n}$ conditioned on $T \cap\left(\begin{array}{c}V \backslash W \\ 2\end{array}\right)=T_{1}$. This is because each such tree $T_{2}$ extends $T_{1}$ to a different spanning tree of $K_{n}$, and each spanning tree where $T \cap\left(\begin{array}{c}V \backslash W \\ 2\end{array}\right)=T_{1}$ can be obtained in this way. Therefore, for a fixed $T_{1}, T_{1} \cup T_{2}$ is a uniformly random spanning tree conditioned on $T \cap\left(\begin{array}{c}V \backslash W \\ 2\end{array}\right)=T_{1}$. Finally, since the distribution of $T_{1}$ is equal to that of a uniformly random spanning tree restricted to $V \backslash W$, $T_{1} \cup T_{2}$ is a uniformly random spanning tree.

Lemma 3.6. The resampling oracle $r_{A}(T)$ applied to a spanning tree satisfying $E_{A}$ does not cause any new event $E_{B}$ such that $B \notin \Gamma^{+}(A)$.

Proof. Note that the only edges that we modify are those incident to $W=V(A)$. Therefore, any new event $E_{B}$ that the operation of $r_{A}$ could cause must be such that $B$ contains an edge incident to $W$ and not contained in $A$. Such an edge shares exactly one vertex with some edge in $A$ and hence $B \sim A$. 


\subsection{Composition of resampling oracles for product spaces}

Suppose we have a product probability space $\Omega=\Omega_{1} \times \Omega_{2} \times \ldots \times \Omega_{N}$, where on each $\Omega_{i}$ we have resampling oracles $r_{i j}$ for events $E_{i j}, j \in \mathcal{E}_{i}$, with respect to a graph $G_{i}$. Our goal is to show that there is a natural way to combine these resampling oracles in order to handle events on $\Omega$ that are obtained by taking intersections of the events $E_{i j}$. The following theorem formalizes this notion.

Theorem 3.7. Let $\Omega_{1}, \ldots, \Omega_{N}$ be probability spaces, where for each $\Omega_{i}$ we have resampling oracles $r_{i j}$ for events $E_{i j}, j \in \mathcal{E}_{i}$ with respect to a graph $G_{i}$. Let $\Omega=\Omega_{1} \times \Omega_{2} \times \ldots \Omega_{N}$ be a product space with the respective product probability measure. For any set $J$ of pairs $(i, j), j \in \mathcal{E}_{i}$ where each $i \in[N]$ appears at most once, define an event $E_{J}$ on $\Omega$ to occur in a state $\omega=\left(\omega_{1}, \ldots, \omega_{N}\right)$ iff $E_{i j}$ occurs in $\omega_{i}$ for each $(i, j) \in J$. Define a graph $G$ on these events by $J \sim J^{\prime}$ iff there exist pairs $(i, j) \in J,\left(i, j^{\prime}\right) \in J^{\prime}$ such that $j \sim j^{\prime}$ in $G_{i}$. Then there exist resampling oracles $r_{J}$ for the events $E_{J}$ with respect to $G$, which are obtained by calling in succession each of the oracles $r_{i j}$ for $(i, j) \in J$.

Proof. For notational simplicity, let us assume that on each $\Omega_{i}$ we have a trivial event $E_{i 0}=\Omega_{i}$ and the respective resampling oracle $r_{i 0}$ is the identity on $\Omega_{i}$. Then we can assume that each collection of events $J$ is in the form $J=\left\{\left(1, j_{1}\right),\left(2, j_{2}\right), \ldots,\left(N, j_{N}\right)\right\}$, where we set $j_{\ell}=0$ for components where there is no event to resample. We define

$$
r_{J}\left(\omega_{1}, \ldots, \omega_{N}\right)=\left(r_{1 j_{1}}\left(\omega_{1}\right), r_{2 j_{2}}\left(\omega_{2}\right), \ldots, r_{N j_{N}}\left(\omega_{N}\right)\right) .
$$

We claim that these are resampling oracles with respect to $G$ as defined in the theorem.

Let us denote by $\mu_{i}$ the probability distribution on $\Omega_{i}$ and by $\mu$ the product distribution on $\Omega$. For the first condition, suppose that $\left.\omega \sim \mu\right|_{E_{J}}$. By the product structure of $\Omega$, this is the same as having $\omega=\left(\omega_{1}, \ldots, \omega_{N}\right)$ where the components are independent and $\left.\omega_{\ell} \sim \mu_{\ell}\right|_{E_{\ell j_{\ell}}}$ for each $\left(\ell, j_{\ell}\right) \in J$, and $\omega_{\ell} \sim \mu_{\ell}$ for components such that $j_{\ell}=0$. By the properties of the resampling oracles $r_{\ell j_{\ell}}$, we have $r_{\ell j_{\ell}}\left(\omega_{\ell}\right) \sim \mu_{\ell}$. Since the resampling oracles are applied with independent randomness for each component, we have

$$
r_{J}(\omega)=\left(r_{1 j_{1}}\left(\omega_{1}\right), r_{2 j_{2}}\left(\omega_{2}\right), \ldots, r_{N j_{N}}\left(\omega_{N}\right)\right) \sim \mu_{1} \times \mu_{2} \times \ldots \times \mu_{N}=\mu .
$$

For the second condition, note that if $\omega \notin E_{J^{\prime}}$ and $r_{J}(\omega) \in E_{J^{\prime}}$, it must be the case that there is $\left(\ell, j_{\ell}\right) \in J$ and $\left(\ell, j_{\ell}^{\prime}\right) \in J^{\prime}$ such that $\omega_{\ell} \notin E_{\ell j_{\ell}^{\prime}}$ and $r_{\ell j_{\ell}}(\omega) \in E_{\ell j_{\ell}^{\prime}}$. However, this is possible only if $j_{\ell} \sim j_{\ell}^{\prime}$ in the graph $G_{\ell}$. By the definition of $G$, this means that $J \sim J^{\prime}$ as well.

As a result, we can extend our resampling oracles to spaces like $N$-tuples of independent random permutations, independent random spanning trees, etc. Such extensions are used in our applications.

\section{Applications}

Let us present a few applications of our framework. Our application to rainbow spanning trees is new, even in the existential sense. Our applications to Latin transversals and rainbow matchings are also new to the best of our knowledge, although they could also have been obtained using the framework of [21] and [2].

\subsection{Rainbow spanning trees}

Given an edge-coloring of $K_{n}$, a spanning tree is called rainbow if each of its edges has a distinct color. The existence of a single rainbow spanning tree is completely resolved by the matroid intersection theorem: It 
can be decided efficiently whether a rainbow spanning tree exists for a given edge coloring, and it can be found efficiently if it exists. However, the existence of multiple edge-disjoint rainbow spanning trees is more challenging. An attractive conjecture of Brualdi and Hollingsworth [11] states that if $n$ is even and $K_{n}$ is properly edge-colored by $n-1$ colors, then the edges can be decomposed into $n / 2$ rainbow spanning trees, each tree using each color exactly once. Until recently, it was only known that every such edge-coloring contains 2 edge-disjoint rainbow spanning trees [5]. In a recent development, it was proved that if every color is used at most $n / 2$ times (which is true for any proper coloring) then there exist $\Omega(n / \log n)$ edgedisjoint rainbow spanning trees [12]. In fact this result seems to be algorithmically efficient, although this was not claimed by the authors. We prove that using our framework, we can find $\Omega(n)$ rainbow spanning trees under a slight strengthening of the coloring assumption.

Theorem 4.1. Given an edge-coloring of $K_{n}$ such that each color appears on at most $\frac{1}{32}\left(\frac{7}{8}\right)^{7} n$ edges, at least $\frac{1}{32}\left(\frac{7}{8}\right)^{7} n$ edge-disjoint rainbow spanning trees exist and can be found in $O\left(n^{4}\right)$ resampling oracle calls with high probability.

This result relies on Theorem 1.3, our algorithmic version of the LLL under the cluster expansion criterion. To obtain the result with high probability, we appeal to a more refined bound that we state in Theorem 5.44. We note that if there is constant multiplicative slack in the assumption on color appearances, the number of resamplings improves to $O\left(n^{2}\right)$, using the result in Theorem 5.44 with constant $\epsilon$ slack.

To prove the existential statement, we simply sample $\frac{1}{32}\left(\frac{7}{8}\right)^{7} n$ independently random spanning trees and hope that they will be (a) pairwise edge-disjoint, and (b) rainbow. This unlikely proposition happens to be true with positive probability, thanks to the LLL and the independence properties of random spanning trees that we mentioned in Section 3.4 Given this setup, our framework implies that we can also find the rainbow trees efficiently.

Proof. We apply our algorithm in the setting of $t$ independent and uniformly random spanning trees $T_{1}, \ldots, T_{t} \subset$ $K_{n}$, with the following two types of bad events:

- $E_{e f}^{i}$ : For each $i \in[t]$ and two edges $e \neq f$ in $K_{n}$ of the same color, $E_{e f}^{i}$ occurs if $\{e, f\} \subset T_{i}$;

- $E_{e}^{i j}$ : For each $i \neq j \in[t]$ and an edge $e$ in $K_{n}, E_{e}^{i j}$ occurs if $e \in T_{i} \cap T_{j}$.

Clearly, if no bad event occurs then the $t$ trees are rainbow and pairwise edge-disjoint.

By (3) the probability of a bad event of the first type is $\operatorname{Pr}\left[E_{e f}^{i}\right]=3 / n^{2}$ if $|e \cup f|=3$ and $\operatorname{Pr}\left[E_{e f}^{i}\right]=$ $4 / n^{2}$ if $|e \cup f|=4$. The probability of a bad event of the second type is $\operatorname{Pr}\left[E_{e}^{i j}\right]=(2 / n)^{2}=4 / n^{2}$, since each of the two trees contains $e$ independently with probability $2 / n$. Hence, the probability of each bad event is upper-bounded by $p=4 / n^{2}$.

In Section 3.4 we constructed a resampling oracle $r_{A}$ for a single spanning tree. By Theorem 3.7 this resampling oracle extends in a natural way to the setting of $t$ independent random spanning trees. In particular, for an event $E_{e f}^{i}$, we define $r_{e f}^{i}$ as an application of the resampling oracle $r_{\{e, f\}}$ to the tree $T_{i}$. For an event $E_{e}^{i j}$, we define $r_{e}^{i j}$ as an application of the resampling oracle $r_{\{e\}}$ independently to the trees $T_{i}$ and $T_{j}$. It is easy to check using Theorem 3.7 that for independent uniformly random spanning trees conditioned on either type of event, the respective resampling oracle generates independent uniformly random spanning trees.

Let us define the following dependency graph; we are somewhat conservative for the sake of simplicity. The graph contains the following kinds of edges:

- $E_{e f}^{i} \sim E_{e^{\prime} f^{\prime}}^{i}$ whenever $e \cup f$ intersects $e^{\prime} \cup f^{\prime}$; 
- $E_{e f}^{i}, E_{e f}^{j} \sim E_{e^{\prime}}^{i j}$ whenever $e^{\prime}$ intersects $e \cup f$;

- $E_{e}^{i j} \sim E_{e^{\prime}}^{i j^{\prime}}, E_{e^{\prime}}^{i^{\prime} j}$ whenever $e^{\prime}$ intersects $e$.

We claim that the resampling oracle for any bad event can cause new bad events only in its neighborhood. This follows from the fact that the resampling oracle affect only the trees relevant to the event (in the superscript), and the only edges modified are those incident to those relevant to the event (in the subscript).

Let us now verify the cluster expansion criterion, introduced as (CLL) in Section 1.4 so that we may apply Theorem 5.44. Let us assume that each color appears on at most $q$ edges, and we generate $t$ random spanning trees. We claim that the neighborhood of each bad event can be partitioned into 4 cliques of size $(n-1)(t-1)$ and 4 cliques of size $(n-1)(q-1)$.

First, let us consider an event of type $E_{e f}^{i}$. The neighborhood of $E_{e f}^{i}$ consists of: (1) events $E_{e^{\prime} f^{\prime}}^{i}$ where $e^{\prime}$ or $f^{\prime}$ shares a vertex with $e \cup f$; these events form 4 cliques, one for each vertex of $e \cup f$, and the size of each clique is at most $(n-1)(q-1)$, since the number of incident edges to a vertex is $n-1$, and the number of other edges of the same color is at most $q-1$. (2) events $E_{e^{\prime}}^{i j}$ where $e^{\prime}$ intersects $e \cup f$; these events form 4 cliques, one for each vertex of $e \cup f$, and each clique has size at most $(n-1)(t-1)$, since its events can be identified with the $(n-1)$ edges incident to a fixed vertex and the remaining $t-1$ trees.

Second, let us consider an event of type $E_{e}^{i j}$. The neighborhood of $E_{e}^{i j}$ consists of: (1) events $E_{e^{\prime} f^{\prime}}^{i}$ and $E_{e^{\prime} f^{\prime}}^{j}$ where $e$ intersects $e^{\prime} \cup f^{\prime}$; these events form 4 cliques, one for each vertex of $e$ and either $i$ or $j$ in the superscript, and the size of each clique is at most $(n-1)(q-1)$ by an argument as above. (2) events $E_{e^{\prime}}^{i^{\prime} j}, E_{e^{\prime}}^{i j^{\prime}}$ where $e^{\prime}$ intersects $e$; these events form 4 cliques, one for each vertex of $e$ and either $i^{\prime} j$ or $i j^{\prime}$ in the superscript. The size of each clique is at most $(n-1)(t-1)$, since the events can be identified with the $(n-1)$ edges incident to a vertex and the remaining $t-1$ trees.

Considering the symmetry of the dependency graph, we set the variables for all events equal to $y_{e f}^{i}=$ $y_{e}^{i j}=y$. The cluster expansion criteria will be satisfied if we set the parameters so that

$$
p \leq \frac{y}{(1+(n-1)(t-1) y)^{4}(1+(n-1)(q-1) y)^{4}} \leq \frac{y}{\sum_{I \subseteq \Gamma^{+}(E), I \in \operatorname{lnd}} y^{I}},
$$

where $E$ denotes either $E_{e f}^{i}$ or $E_{e}^{i j}$. The second inequality holds due to the structure of the neighborhood of each event that we described above. We set $y=\beta p=4 \beta / n^{2}$ and assume $t \leq \gamma n, q \leq \gamma n$. The reader can verify that with the settings $\beta=\left(\frac{8}{7}\right)^{8}$ and $\gamma=\frac{1}{32}\left(\frac{7}{8}\right)^{7}$, we get $\frac{\beta}{(1+4 \gamma \beta)^{8}}=1$. Therefore,

$$
p \leq \frac{\beta p}{(1+4 \gamma \beta)^{8}} \leq \frac{y}{(1+(n-1)(t-1) y)^{4}(1+(n-1)(q-1) y)^{4}}
$$

which verifies the assumption of Theorem 5.44 Theorem5.44 implies that MaximalSetResample terminates after $O\left(\left(\sum y_{e f}^{i}+\sum y_{e}^{i j}\right)^{2}\right)$ resampling oracle calls with high probability. The total number of events here is $O\left(t q n^{2}\right)=O\left(n^{4}\right)$ and for each event the respective variable is $y=O\left(1 / n^{2}\right)$. Therefore, the expected number of resampling oracle calls is $O\left(n^{4}\right)$.

\subsection{Rainbow matchings}

Given an edge-coloring of $K_{2 n}$, a perfect matching is called rainbow if each of its edges has a distinct color. This can be viewed as a non-bipartite version of the problem of Latin transversals. It is known that given any proper $(2 n-1)$-edge-coloring of $K_{2 n}$ (where each color forms a perfect matching), there exists a rainbow perfect matching [38]. However, finding rainbow matchings algorithmically is more difficult. Achlioptas 
and Iliopoulos [2] showed how to find a rainbow matching in $K_{2 n}$ efficiently when each color appears on at most $\gamma n$ edges, $\gamma<\frac{1}{2 e} \simeq 0.184$. Our result is that we can do this for $\gamma=\frac{27}{128} \simeq 0.211$. The improvement comes from the application of the "cluster expansion" form of the local lemma, which is still efficient in our framework. (We note that an updated version of the Achlioptas-Iliopoulos framework [3] also contains this result.)

Theorem 4.2. Given an edge-coloring of $K_{2 n}$ where each color appears on at most $\frac{27}{128} n$ edges, a rainbow perfect matching exists and can be found in $O\left(n^{2}\right)$ resampling oracle calls with high probability.

In fact, we can find many disjoint rainbow matchings - up to a linear number, if we replace $\frac{27}{128}$ above by a smaller constant.

Theorem 4.3. Given an edge-coloring of $K_{2 n}$ where each color appears on at most $\frac{7^{7}}{8^{8}} n$ edges, at least $\frac{7^{7}}{8^{8}} n$ edge-disjoint rainbow perfect matchings exist and can be found in $O\left(n^{4}\right)$ resampling oracle calls whp.

We postpone the proof to Section 4.3, since it follows from our result for Latin transversals.

Proof of Theorem 4.2. We apply our algorithm in the setting of uniformly random perfect matchings $M \subset$ $K_{2 n}$, with the following bad events (identical to the setup in [2]): For every pair of edges $e, f$ of the same color, $E_{e f}$ occurs if $\{e, f\} \subset M$. If no bad event $E_{e f}$ occurs then $M$ is a rainbow matching. We also define the following dependency graph: $E_{e f} \sim E_{e^{\prime} f^{\prime}}$ unless $e, f, e^{\prime}, f^{\prime}$ are four disjoint edges. Note that this is more conservative than the dependency graph we considered in Section 3.3, where two events are only connected if they do not form a matching together. The more conservative definition will simplify our analysis. In any case, our resampling oracle is consistent with this lopsidependency graph in the sense that resampling $E_{e f}$ can only cause new events $E_{e^{\prime} f^{\prime}}$ such that $E_{e f} \sim E_{e^{\prime} f^{\prime}}$. We show that this setup satisfies the criteria of the cluster expansion lemma.

Let $q=\frac{27}{128} n, p=\frac{1}{(2 n-1)(2 n-3)}$ and $y=\left(\frac{4}{3}\right)^{4} p$. Consider the neighborhood of a bad event $\Gamma\left(E_{e f}\right)$. It contains all events $E_{e^{\prime} f^{\prime}}$ such that there is some intersection among the edges $e, f, e^{\prime}, f^{\prime}$. Such events can be partitioned into 4 cliques: for each vertex $v \in e \cup f$, let $\mathcal{Q}_{v}$ denote all the events $E_{e^{\prime} f^{\prime}}$ such that $v \in e^{\prime}$ and $f^{\prime}$ has the same color as $e^{\prime}$. The number of edges $e^{\prime}$ incident to $v$ is $2 n-1$, and for each of them, the number of other edges of the same color is by assumption at most $q-1$. Therefore, the size of $\mathcal{Q}_{v}$ is at most $(q-1)(2 n-1)$.

In the following, we use the short-hand notation $y^{I}=\prod_{i \in I} y_{i}$. Consider the assumptions of the cluster expansion lemma: for each event $E_{e f}$, we should have

$$
\operatorname{Pr}\left[E_{e f}\right] \leq \frac{y_{e f}}{\sum_{I \subseteq \Gamma^{+}\left(E_{e f}\right), I \in \operatorname{lnd}} y^{I}}
$$

We have $\operatorname{Pr}\left[E_{e f}\right]=p=\frac{1}{(2 n-1)(2 n-3)}$. By symmetry, we set all the variables $y_{e f}$ to the same value, $y_{e f}=y=\left(\frac{4}{3}\right)^{4} p$. Note that an independent subset of $\Gamma^{+}\left(E_{e f}\right)$ can contain at most 1 event from each clique $\mathcal{Q}_{v}$. (The event $E_{e f}$ itself is also contained in these cliques.) Therefore,

$$
\sum_{I \subseteq \Gamma^{+}\left(E_{e f}\right), I \in \text { Ind }} y^{I} \leq \prod_{v \in e \cup f}\left(1+\sum_{E_{e^{\prime} f^{\prime}} \in \mathcal{Q}_{v}} y_{e^{\prime} f^{\prime}}\right) \leq(1+(q-1)(2 n-1) y)^{4} .
$$

The reader can verify that $\sum_{I \subseteq \Gamma^{+}\left(E_{e f}\right), I \in \operatorname{lnd}} y^{I} \leq(1+(q-1)(2 n-1) y)^{4} \leq\left(1+\frac{27}{64} n^{2}\left(\frac{4}{3}\right)^{4} /(2 n)^{2}\right)^{4}=\left(\frac{4}{3}\right)^{4}$. Therefore,

$$
\frac{y}{\sum_{I \subseteq \Gamma^{+}\left(E_{e f}\right), I \in \operatorname{lnd}} y^{I}} \geq p
$$


which is the assumption of Theorem 5.44. By Theorem 5.44, MaximalSetResample with the resampling oracle for matchings and the dependency graph defined above will find a rainbow perfect matching in time $O\left(\sum_{E_{e f}} y_{e f} \sum_{E_{e f}} \log \left(1+y_{e f}\right)\right)=O\left(\left(\sum_{E_{e f}} y_{e f}\right)^{2}\right)$ with high probability. The number of bad events $E_{e f}$ is $O\left(n^{3}\right)$, because each color class has $O(n)$ edges so the number of edge pairs of equal color is $O\left(n^{3}\right)$. We have $y_{e f}=O\left(1 / n^{2}\right)$, and hence the total number of resamplings is $O\left(n^{2}\right)$ with high probability.

\subsection{Latin transversals}

A Latin transversal in an $n \times n$ matrix $A$ is a permutation $\pi \in S_{n}$ such that the entries $A_{i, \pi(i)}$ ("colors") are distinct for $i=1,2, \ldots, n$. In other words, it is a set of distinct entries, exactly one in each row and one in each column. It is easy to see that this is equivalent to a bipartite version of the rainbow matching problem: $A_{i j}$ is the color of the edge $(i, j)$ and we are looking for a perfect bipartite matching where no color appears twice. It is a classical application of the Lovász Local Lemma that if no color appears more than $\frac{1}{4 e} n$ times in $A$ then there exists a Latin transversal [16]. An improvement of this result is that if no color appears more than $\frac{27}{256} n$ times in $A$ then a Latin transversal exists [8]; this paper introduced the "cluster expansion" strengthening of the local lemma. (Note that $\frac{27}{256}=\frac{3^{3}}{4^{4}}$.) These results were made algorithmically efficient by the work of Harris and Srinivasan [21].

Beyond finding one Latin transversal, one can ask whether there exist multiple disjoint Latin transversals. A remarkable existential result was proved by Alon, Spencer and Tetali [7]: If $n=2^{k}$ and each color appears in $A$ at most $\epsilon n$ times ( $\epsilon=10^{-10^{10}}$ in their proof), then $A$ can be partitioned into $n$ disjoint Latin transversals. Here, we show how to find a linear number of Latin transversals algorithmically.

Theorem 4.4. For any $n \times n$ matrix $A$ where each color appears at most $\frac{7^{7}}{8^{8}} n$ times, there exist at least $\frac{7^{7}}{8^{8}} n$ disjoint Latin transversals, and they can be found in $O\left(n^{4}\right)$ resampling oracle calls w.h.p.

We note that again, if there is constant multiplicative slack in the assumption on color appearances, the number of resamplings improves to $O\left(n^{2}\right)$. This also implies Theorem 4.3 as a special case: For an edge-coloring of $K_{2 n}$ where no color appears more than $\frac{7^{7}}{8^{8}} n$ times, let us label the vertices arbitrarily $\left(u_{1}, \ldots, u_{n}, v_{1}, \ldots, v_{n}\right)$ construct a matrix $A$ where $A_{i j}$ is the color of the edge $\left(u_{i}, v_{j}\right)$. If no color appears more than $\frac{7^{7}}{8^{8}} n$ times, by Theorem 4.4 we can find $\frac{7^{7}}{8^{8}} n$ Latin transversals; these correspond to rainbow matchings in $K_{2 n}$.

Our approach to proving Theorem 4.4 is similar to the proof of Theorem 4.1 sample $\frac{7^{7}}{8^{8}} n$ independently random permutations and hope that they will be (a) disjoint, and (b) Latin. For reasons similar to Theorem 4.1, the local lemma works out and our framework makes this algorithmic.

Proof. Let $t=\frac{7^{7}}{8^{8}} n$ and let $\pi_{1}, \ldots, \pi_{t}$ be independently random permutations on $[n]$. We consider the following two types of bad events:

- $E_{e f}^{i}$ : For each $i \in[t]$ and $e=(u, v), f=(x, y) \in[n] \times[n]$ such that $u \neq v, x \neq y, A_{u v}=A_{x y}$, the event $E_{e f}^{i}$ occurs if $\pi_{i}(u)=v$ and $\pi_{i}(x)=y$;

- $E_{e}^{i j}$ : For each $i \neq j \in[t]$ and $e=(u, v) \in[n] \times[n]$, the event $E_{e}^{i j}$ occurs if $\pi_{i}(u)=\pi_{j}(u)=v$.

Clearly, if none of these events occurs then the permutations $\pi_{1}, \ldots, \pi_{t}$ correspond to pairwise disjoint Latin transversals. The probability of a bad event of the first type is $\operatorname{Pr}\left[E_{e f}^{i}\right]=\frac{1}{n(n-1)}$ and the probability for the second type is $\operatorname{Pr}\left[E_{e}^{i j}\right]=\frac{1}{n^{2}}$. Thus the probability of each bad event is at most $p=\frac{1}{n(n-1)}$. 
It will be convenient to think of the pairs $e=(x, y) \in[n] \times[n]$ as edges in a bipartite complete graph. As we proved in Section 3.2 the resampling oracle for permutations is consistent with the following lopsidependency graph graph.

- $E_{e f}^{i} \sim E_{e^{\prime} f^{\prime}}^{i}$ whenever there is some intersection between the edges $e, f$ and $e^{\prime}, f^{\prime}$;

- $E_{e f}^{i}, E_{e f}^{j} \sim E_{e^{\prime}}^{i j}$ whenever there is some intersection between $e^{\prime}$ and $e, f$;

- $E_{e}^{i j} \sim E_{e^{\prime}}^{i j^{\prime}}, E_{e^{\prime}}^{i^{\prime} j}$ whenever $e^{\prime}$ intersects $e$.

By Lemma 3.2, the resampling oracle for a given event never causes a new event except in its neighborhood.

Let us now verify the cluster expansion criteria. The counting here is quite similar to the proof of Theorem 4.1, so we skim over some details. The neighborhood of each event $E_{e f}^{i}$ consist of 8 cliques: 4 cliques of events of type $E_{e^{\prime} f^{\prime}}^{i}$ and 4 cliques of events of type $E_{e}^{i j}$, corresponding in each case to the 4 vertices of $e \cup f$. In the first case, each clique has at most $n(q-1)$ events, determined by selecting an incident edge and another edge of the same color. In the second case, each clique has at most $n(t-1)$ events, determined by selecting an incident edge and another permutation.

The neighborhood of each event $E_{e}^{i j}$ also consists of 8 cliques: 4 cliques of events $E_{e^{\prime} f^{\prime}}^{i}$ or $E_{e^{\prime} f^{\prime}}^{j}$, corresponding to the choice of either $i$ or $j$ in the superscript, and one of the two vertices of $e$. The size of each clique is at most $n(q-1)$, determined by choosing an incident edge and another edge of the same color. Then, we have 4 cliques of events $E_{e^{\prime}}^{i j^{\prime}}$ or $E_{e^{\prime}}^{i^{\prime} j}$, determined by switching either $i^{\prime}$ or $j^{\prime}$ in the superscript, and choosing one of the vertices of $e$. The size of each clique is at most $n(t-1)$, determined by choosing an incident edge and a new permutation in the superscript.

As a consequence, the cluster expansion criterion here is almost exactly the same as in the case of Theorem 4.1 .

$$
p \leq \frac{y}{(1+n(t-1) y)^{4}(1+n(q-1) y)^{4}} .
$$

We have $p=\frac{1}{n(n-1)}$ here and we set $y=\beta p$. For $t, q \leq \gamma n$, it's enough to satisfy $\frac{\beta}{(1+\beta \gamma)^{8}} \geq 1$, which is achieved by $\beta=\left(\frac{8}{7}\right)^{8}$ and $\gamma=\frac{7^{7}}{8^{8}}$. Therefore, Theorem 5.44 implies that MaximalSetResample will terminate within $O\left(\left(\sum y_{e f}^{i}+\sum y_{e}^{i j}\right)^{2}\right)=O\left(n^{4}\right)$ resampling oracle calls with high probability.

\section{Analysis of the algorithm}

Here we provide the analysis of our algorithm and the proofs of our main theorems. In Section 5.1, we begin with the basic notions necessary for our analysis and a coupling argument which forms the basis of all our algorithmic results. In Section 5.2, we prove a weaker form of Theorem 1.2 under the assumption that the (GLL) criterion holds with some slack. In Section 5.3, we introduce the independence polynomial of a graph and summarize its fundamental properties that are important for our analysis. In Section 5.4, we prove that our algorithm is efficient if Shearer's criterion is satisfied with an $\epsilon$ slack. In Section 5.5, we show that in some sense this assumption is not necessary, because every point satisfying Shearer's criterion has some slack available, and we quantify how large this slack is. Finally, we return to the weaker (but more practical) variants of the local lemma: the (GLL) and (CLL) criteria. We present new combinatorial connections between these criteria and Shearer's criterion, which in turn imply our main results on the efficiency of our algorithm under the (GLL) and (CLL) criteria (in Sections 5.6 and 5.7 respectively). 


\subsection{Stable set sequences and the coupling argument}

An important notion in our analysis is that of stable set sequences. We note that this concept originated in the work of Kolipaka and Szegedy [24] which builds on Shearer's work [36]. There are some similarities but also differences in how this concept is applied here: most notably, our stable set sequences grow forward in time, while the stable set sequences in [24] grow backward in time (which is similar to the Moser-Tardos analysis [30]).

Definition 5.1. One execution of the outer repeat loop in MaximalSetResample is called an iteration. For a sequence of non-empty sets $\mathcal{I}=\left(I_{1}, \ldots, I_{t}\right)$, we say that the algorithm follows $\mathcal{I}$ if $I_{s}$ is the set resampled in iteration $s$ for $1 \leq s<t$, and $I_{t}$ is a set of the first $m$ events resampled in iteration $t$ for some $m \geq 1$ ( $a$ prefix of the maximal independent set constructed in iteration $t$ ).

Recall that Ind $=\operatorname{lnd}(G)$ denotes the independent sets (including the empty set) in the graph under consideration.

Definition 5.2. $\mathcal{I}=\left(I_{1}, I_{2}, \ldots, I_{t}\right)$ is called a stable set sequence if $I_{1}, \ldots, I_{t} \in \operatorname{Ind}(G)$ and $I_{s+1} \subseteq \Gamma^{+}\left(I_{s}\right)$ for each $1 \leq s<t$. We call the sequence $\mathcal{I}$ proper if each independent set $I_{s}$ is nonempty.

Note that if $I_{s}=\emptyset$ for some $s$, then $I_{t}=\emptyset$ for all $t>s$. Therefore, the nonempty sets always form a prefix of the stable set sequence. Formally, we consider an empty sequence also a stable set sequence, of length 0 .

Lemma 5.3. If MaximalSetResample follows a sequence $\mathcal{J}=\left(J_{1}, \ldots, J_{t}\right)$, then $\mathcal{J}$ is a stable set sequence.

Proof. By construction, the set $J_{s}$ chosen in each iteration is independent in $G$. For each $i \in J_{s}$, we execute the resampling oracle $r_{i}$. Recall that $r_{i}$ executed on a satisfied event $E_{i}$ can only cause new events in the neighborhood $\Gamma^{+}(i)$ (and this neighborhood is not explored again until the following iteration). Since $J_{s}$ is a maximal independent set of satisfied events, all the events satisfied in the following iteration are neighbors of some event in $J_{s}$, i.e., $J_{s+1} \subseteq \Gamma^{+}\left(J_{s}\right)$. In the last iteration, this also holds for a subset of the resampled events.

We use the following notation: For $i \in[n], p_{i}=\operatorname{Pr}_{\mu}\left[E_{i}\right]$. For $S \subseteq[n], p^{S}=\prod_{i \in S} p_{i}$. For a stable set sequence $\mathcal{I}=\left(I_{1}, \ldots, I_{t}\right), p_{\mathcal{I}}=\prod_{s=1}^{t} p^{I_{s}}$. We relate stable set sequences to executions of the algorithm by the following coupling argument. Although the use of stable set sequences is inspired by [24], their coupling argument is different due to its backward-looking nature (similar to [30]), and their restriction to the variable model.

Lemma 5.4. For any proper stable set sequence $\mathcal{I}=\left(I_{1}, I_{2}, \ldots, I_{t}\right)$, the probability that the MaximalSetResample algorithm follows $\mathcal{I}$ is at most $p_{\mathcal{I}}$.

Proof. Given $\mathcal{I}=\left(I_{1}, I_{2}, \ldots, I_{t}\right)$, let us consider the following " $\mathcal{I}$-checking" random process. We start with a random state $\omega \sim \mu$. In iteration $s$, we process the events of $I_{s}$ in the ascending order of their indices. For each $i \in I_{s}$, we check whether $\omega$ satisfies $E_{i}$; if not, we terminate. Otherwise, we apply the resampling oracle $r_{i}$ and replace $\omega$ by $r_{i}(\omega)$. We continue for $s=1,2, \ldots, t$. We say that the $\mathcal{I}$-checking process succeeds if every event is satisfied when checked and the process runs until the end.

By induction, the state $\omega$ after each resampling oracle call is distributed according to $\mu$ : Assuming this was true in the previous step and conditioned on $E_{i}$ satisfied, we have $\left.\omega \sim \mu\right|_{E_{i}}$. By assumption, the resampling oracle $r_{i}$ removes this conditioning and produces again a random state $r_{i}(\omega) \sim \mu$. Therefore, 
whenever we check event $E_{i}$, it is satisfied with probability $\operatorname{Pr}_{\mu}\left[E_{i}\right]$ (conditioned on the past). By a telescoping product of conditional probabilities, the probability that the $\mathcal{I}$-checking process succeeds is exactly $\prod_{s=1}^{t} \prod_{i \in I_{s}} \operatorname{Pr}_{\mu}\left[E_{i}\right]=\prod_{s=1}^{t} p^{I_{s}}=p_{\mathcal{I}}$.

To conclude, we argue that the probability that MaximalSetResample follows the sequence $\mathcal{I}$ is at most the probability that the $\mathcal{I}$-checking process succeeds. To see this, suppose that we couple MaximalSetResample and the $\mathcal{I}$-checking process, so they use the same source of randomness. In each iteration, if MaximalSetResample includes $i$ in $J_{t}$, it means that $E_{i}$ is satisfied. Both procedures apply the resampling oracle $r_{I}(\omega)$ and by coupling the distribution in the next iteration is the same. Therefore, the event that MaximalSetResample follows the sequence $\mathcal{I}$ is contained in the event that the $\mathcal{I}$-checking process succeeds, which happens with probability $p_{\mathcal{I}}$.

We emphasize that we do not claim that the distribution of the current state $\omega \in \Omega$ is $\mu$ after each resampling oracle call performed by the MaximalSetResample algorithm. This would mean that the algorithm is not making any progress in its search for a state avoiding all events. It is only the $\mathcal{I}$-checking process that has this property.

Definition 5.5. Let Stab denote the set of all stable set sequences and Prop the set of proper stable set sequences. Let us denote by $\operatorname{Stab}_{\ell}$ the set of stable set sequences $\left(I_{1}, \ldots, I_{\ell}\right)$ of length $\ell$, and by $\operatorname{Stab}_{\ell}(J)$ the subset of $\mathrm{Stab}_{\ell}$ such that the first set in the sequence is J. Similarly, denote by $\mathrm{Prop}_{\ell}$ the set of proper stable set sequences of length $\ell$, and by $\operatorname{Prop}(J)$ the subset of Prop such that the first set in the sequence is $J$. For $\mathcal{I}=\left(I_{1}, \ldots, I_{t}\right) \in$ Prop, let us call $\sigma(\mathcal{I})=\sum_{s=1}^{t}\left|I_{s}\right|$ the total size of the sequence.

Lemma 5.6. The probability that MaximalSetResample runs for at least $\ell$ iterations is at most $\sum_{\mathcal{I} \in \operatorname{Prop}_{\ell}} p_{\mathcal{I} \text {. }}$ The probability that MaximalSetResample resamples at least $s$ events is at most $\sum_{\mathcal{I} \in \operatorname{Prop}: \sigma(\mathcal{I})=s} p_{\mathcal{I}}$.

Proof. If the algorithm runs for at least $\ell$ iterations, it means that it follows some proper sequence $\mathcal{I}=$ $\left(I_{1}, I_{2}, \ldots, I_{\ell}\right)$. By Lemma 5.4, the probability that the algorithm follows a particular stable set sequence $\mathcal{I}$ is at most $p_{\mathcal{I}}$. By the union bound, the probability that the algorithm runs for at least $\ell$ iterations is at most $\sum_{\mathcal{I}=\left(I_{1}, \ldots, I_{\ell}\right) \in \operatorname{Prop}} p_{\mathcal{I}}$.

Similarly, if the algorithm resamples at least $s$ events, it means that it follows some proper sequence $\mathcal{I}$ of total size $\sigma(\mathcal{I})=s$. By the union bound, the probability of resampling at least $s$ events is upper-bounded by $\sum_{\mathcal{I} \in \operatorname{Prop}: \sigma(\mathcal{I})=s} p_{\mathcal{I}}$.

We note that these bounds could be larger than 1 and thus vacuous. The events that "the algorithm follows $\mathcal{I}=\left(I_{1}, \ldots, I_{\ell}\right)$ " are disjoint for different sequences of fixed total size $\sigma(\mathcal{I})$, while they could overlap for a fixed length $\ell$ (because we can take $I_{\ell}$ to be different prefixes of the sequence of events resampled in iteration $t$ ). In any case, the upper bound of $p_{\mathcal{I}}$ on each of the events could be quite loose.

\subsection{A simple analysis: the General Lovász Lemma criterion, with slack}

In this section we will analyze the algorithm under the assumption that the (GLL) criterion holds with some "slack". This idea of exploiting slack has appeared in previous work, e.g., [30, 13, 20, 24]. This analysis proves only a weaker form of Theorem 1.2. The full proof, which removes the assumption of slack, appears in Section 5.6

To begin, let us prove the following (crude) bound on the expected number of iterations. We note that this bound is typically exponentially large. 
Lemma 5.7. Provided that the $p_{i}$ satisfy the (GLL) criterion, $p_{i} \leq x_{i} \prod_{j \in \Gamma(i)}\left(1-x_{j}\right)$, we have

$$
\sum_{\mathcal{I} \in \text { Prop }} p_{\mathcal{I}} \leq \prod_{i=1}^{n} \frac{1}{1-x_{i}}
$$

Proof. It will be convenient to work with sequences of fixed length, where we pad by empty sets if necessary. Note that by definition this does not change the value of $p_{\mathcal{I}}$ : e.g., $p_{\left(I_{1}, I_{2}\right)}=p_{\left(I_{1}, I_{2}, \emptyset, \ldots, \emptyset\right)}$. Recall that $\operatorname{Stab}_{\ell}(J)$ denotes the set of all stable set sequences of length $\ell$ where the first set is $J$. We show the following statement by induction on $\ell$ : For any $J \in$ Ind and any $\ell \geq 1$,

$$
\sum_{\mathcal{I} \in \operatorname{Stab}_{\ell}(J)} p_{\mathcal{I}} \leq \prod_{j \in J} \frac{x_{j}}{1-x_{j}}
$$

This is true for $\ell=1$, since $p_{(J)}=p^{J} \leq \prod_{j \in J} x_{j}$ by the LLL assumption. Let us consider the expression for $\ell+1$. We have

$$
\sum_{\mathcal{I}^{\prime} \in \operatorname{Stab}_{\ell+1}(J)} p_{\mathcal{I}^{\prime}}=p^{J} \sum_{J^{\prime} \subseteq \Gamma^{+}(J)} \sum_{\mathcal{I} \in \operatorname{Stab}_{\ell}\left(J^{\prime}\right)} p_{\mathcal{I}} \leq p^{J} \sum_{J^{\prime} \subseteq \Gamma^{+}(J)} \prod_{i \in J^{\prime}} \frac{x_{i}}{1-x_{i}}
$$

by the inductive hypothesis. This can be simplified using the following identity:

$$
\prod_{i \in \Gamma^{+}(J)}\left(1+\alpha_{i}\right)=\sum_{I_{1} \subseteq \Gamma^{+}(J)} \prod_{i \in I_{1}} \alpha_{i}
$$

We use this with $\alpha_{i}=\frac{x_{i}}{1-x_{i}}$. Therefore,

$$
\sum_{\mathcal{I}^{\prime} \in \operatorname{Stab}_{\ell+1}(J)} p_{\mathcal{I}^{\prime}} \leq p^{J} \prod_{i \in \Gamma^{+}(J)}\left(1+\frac{x_{i}}{1-x_{i}}\right)=p^{J} \prod_{i \in \Gamma^{+}(J)} \frac{1}{1-x_{i}} .
$$

Now we use the LLL assumption:

$$
p^{J}=\prod_{i \in J} p_{i} \leq \prod_{i \in J}\left(x_{i} \prod_{j \in \Gamma(i)}\left(1-x_{j}\right)\right) \leq \prod_{i \in J} x_{i} \prod_{j \in \Gamma^{+}(J) \backslash J}\left(1-x_{j}\right)
$$

because each element of $\Gamma^{+}(J) \backslash J$ appears in $\Gamma(i)$ for at least one $i \in J$. We conclude that

$$
\sum_{\mathcal{I}^{\prime} \in \operatorname{Stab}_{\ell}(J)} p_{\mathcal{I}^{\prime}} \leq \prod_{i \in J} x_{i} \prod_{j \in \Gamma^{+}(J) \backslash J}\left(1-x_{j}\right) \cdot \prod_{i^{\prime} \in \Gamma^{+}(J)} \frac{1}{1-x_{i^{\prime}}}=\prod_{i \in J} \frac{x_{j}}{1-x_{j}} .
$$

This proves (4).

Adding up over all sets $J \subseteq[n]$, we again use (5) to obtain

$$
\sum_{\mathcal{I} \in \operatorname{Stab}_{\ell}} p_{\mathcal{I}} \leq \sum_{J \subseteq[n]} \prod_{j \in J} \frac{x_{j}}{1-x_{j}}=\prod_{i=1}^{n}\left(1+\frac{x_{i}}{1-x_{i}}\right)=\prod_{i=1}^{n} \frac{1}{1-x_{i}} .
$$

As we argued above, this can be written equivalently as

$$
\sum_{k=1}^{\ell} \sum_{\mathcal{I} \in \text { Prop }} p_{\mathcal{I}} \leq \prod_{i=1}^{n} \frac{1}{1-x_{i}}
$$

Since this is true for every $\ell$, and the left-hand-side is non-increasing in $\ell$, the sequence as $\ell \rightarrow \infty$ has a limit and the bound still holds in the limit. 
The following is our first concrete result: our algorithm is efficient if (GLL) is satisfied with a slack.

Theorem 5.8. If (GLL) is satisfied with a slack of $\epsilon$, i.e.

$$
\operatorname{Pr}_{\mu}\left[E_{i}\right] \leq(1-\epsilon) x_{i} \prod_{j \in \Gamma(i)}\left(1-x_{j}\right)
$$

then with probability $1-e^{-t}$ MaximalSetResample resamples at most $\frac{1}{\epsilon}\left(t+\sum_{i=1}^{n} \ln \frac{1}{1-x_{i}}\right)$ events.

Proof. By Lemma 5.6, the probability that MaximalSetResample resamples more than $s$ events is at most $\sum_{\mathcal{I} \in \operatorname{Prop}: \sigma(\mathcal{I})=\lceil s\rceil} p_{\mathcal{I}}$ where $p_{\mathcal{I}}$ is the product of $p_{i}=\operatorname{Pr}_{\mu}\left[E_{i}\right]$ over all events in the sequence $\mathcal{I}$. By the slack assumption, we have $p_{i} \leq(1-\epsilon) p_{i}^{\prime}$ and $p_{\mathcal{I}} \leq(1-\epsilon)^{\sigma(\mathcal{I})} p_{\mathcal{I}}^{\prime}$, where $p_{i}^{\prime}=x_{i} \prod_{j \in \Gamma(i)}\left(1-x_{j}\right)$. Using Lemma5.7, we obtain

$$
\sum_{\substack{\mathcal{I} \in \operatorname{Prop} \\ \sigma(\mathcal{I})=\lceil s\rceil}} p_{\mathcal{I}} \leq(1-\epsilon)^{s} \sum_{\mathcal{I} \in \text { Prop }} p_{\mathcal{I}}^{\prime} \leq e^{-\epsilon s} \prod_{i=1}^{n} \frac{1}{1-x_{i}} .
$$

For $s=\frac{1}{\epsilon}\left(t+\sum_{i=1}^{n} \ln \frac{1}{1-x_{i}}\right)$, we obtain

$$
\sum_{\substack{\mathcal{I} \in \operatorname{Prop} \\ \sigma(\mathcal{I})=\lceil s\rceil}} p_{\mathcal{I}} \leq e^{-\epsilon s} \prod_{i=1}^{n} \frac{1}{1-x_{i}} \leq e^{-t} .
$$

Therefore, the probability of resampling more than $s$ events is at most $e^{-t}$.

\subsection{Preliminaries on Shearer's criterion}

In this section we discuss a strong version of the local lemma due to Shearer [36]. Shearer's lemma is based on certain forms of the multivariate independence polynomial. We recall that $p^{I}$ denotes $\prod_{i \in I} p_{i}$.

Definition 5.9. Given a graph $G$ and values $p_{1}, \ldots, p_{n}$, define for each $S \subseteq[n]$

$$
q_{S}=q_{S}(p)=\sum_{\substack{I \in \operatorname{Ind} \\ S \subseteq I}}(-1)^{|I \backslash S|} p^{I}
$$

Note that $q_{S}=0$ for $S \notin$ Ind. An alternative form of these polynomials that is also useful is obtained by summing over subsets of $S$.

Definition 5.10. Given a graph $G$ and values $p_{1}, \ldots, p_{n}$, define

$$
\breve{q}_{S}=\breve{q}_{S}(p)=\sum_{\substack{I \in \operatorname{Ind} \\ I \subseteq S}}(-1)^{|I|} p^{I} .
$$

The following set plays a fundamental role.

Definition 5.11. Given a graph $G$, the Shearer region is the semialgebraic set

$$
\begin{aligned}
\mathcal{S} & =\left\{p \in(0,1)^{n}: \forall I \in \operatorname{lnd}, q_{I}(p)>0\right\} \\
& =\left\{p \in(0,1)^{n}: \forall S \subseteq[n], \breve{q}_{S}(p)>0\right\}
\end{aligned}
$$


The equivalence between (7a) and (7b) is proven below in Claim 5.19

Shearer's Lemma can be stated as follows.

Lemma 5.12 (Shearer [36]). Let $G$ be a lopsidependency graph for the events $E_{1}, \ldots, E_{n}$. Let $p_{i}=$ $\operatorname{Pr}_{\mu}\left[E_{i}\right] \in(0,1)$. If $p \in \mathcal{S}$ then $\operatorname{Pr}_{\mu}\left[\bigcap_{i=1}^{n} \overline{E_{i}}\right] \geq q_{\emptyset}$.

It is known that Shearer's Lemma implies Theorem 1.1, as we will see in Section 5.6, and in fact gives the tight criterion under which all events can be avoided for a given dependency graph $G$. The polynomials $q_{S}(p)$ and $\breve{q}_{S}(p)$ have a natural interpretation in the Shearer region: There is a "tight instance" where $q_{S}(p)$ is the probability that the set of occurring events is exactly $S$, and $\breve{q}_{S}(p)$ is the probability that none of the events in $S$ occur. In particular, $q_{\emptyset}(p)=\breve{q}_{[n]}(p)$ is exactly the probability that no event occurs. (See [36] for more details.)

\subsubsection{Properties of independence polynomials}

In this section we summarize some of the important properties of these polynomials, most of which may be found in earlier work. Since some of the proofs are not easy to recover due to different notation and/or their analytic nature (in case of [35]), we provide short combinatorial proofs for completeness.

Claim 5.13 (The "fundamental identity". Shearer [36], Scott-Sokal [35, Eq. (3.5)]). For any $a \in S$, we have

$$
\breve{q}_{S}=\breve{q}_{S \backslash\{a\}}-p_{a} \cdot \breve{q}_{S \backslash \Gamma^{+}(a)} \cdot
$$

Proof. Every independent set $I \subseteq S$ either contains $a$ or does not. In addition, if $a \in I$ then $I$ is independent iff $I \backslash\{a\}$ is an independent subset of $S \backslash \Gamma^{+}(a)$.

Claim 5.14 (Shearer [36], Scott-Sokal [35, Eq. (2.52)]). For every $S \subseteq[n]$,

$$
\breve{q}_{S}=\sum_{Y \subseteq[n] \backslash S} q_{Y}
$$

Proof. By definition of $q_{Y}$,

$$
\sum_{Y \subseteq[n] \backslash S} q_{Y}=\sum_{Y \subseteq[n] \backslash S} \sum_{\substack{I \in \text { Ind } \\ Y \subseteq I}}(-1)^{|I \backslash Y|} p^{I}=\sum_{I \in \operatorname{lnd}} p^{I} \sum_{Y \subseteq I \backslash S}(-1)^{|I \backslash Y|} .
$$

If $I \backslash S \neq \emptyset$ then the last alternating sum is zero. Therefore, the sum simplifies to $\sum_{I \in \operatorname{lnd}: I \subseteq S}(-1)^{|I|} p^{I}=\breve{q}_{S}$ as required.

Claim 5.15 (Shearer [36]).

$$
\sum_{J \in \text { Ind }} q_{J}=\sum_{S \subseteq[n]} q_{S}=1
$$

Proof. Set $S=\emptyset$ in Claim 5.14 and use the fact that $\breve{q}_{\emptyset}=1$.

Claim 5.16 (Scott-Sokal [35, Eq. (2.48)]). For $I \in$ Ind,

$$
q_{I}=p^{I} \cdot \breve{q}_{[n] \backslash \Gamma^{+}(I)} .
$$


Proof. Given $I \in$ Ind, each independent set $J \supseteq I$ can be written uniquely as $J=I \cup K$ where $K$ is independent and $K \cap \Gamma^{+}(I)=\emptyset$. So,

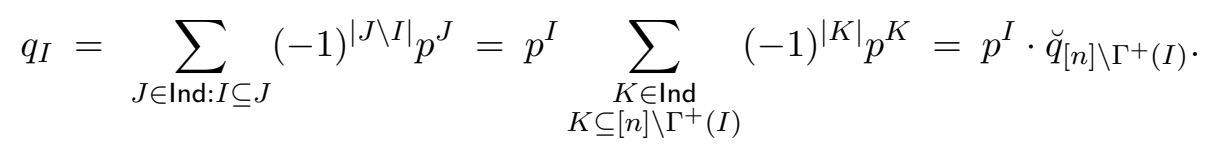

Lemma 5.17 (Kolipaka-Szegedy [24, Lemma 15]). For any $I \in$ Ind

$$
q_{I}=p^{I} \cdot \sum_{S \subseteq \Gamma^{+}(I)} q_{S}
$$

Proof. By Claim 5.16 and Claim 5.14, we have $q_{I}=p^{I} \cdot \breve{q}_{[n] \backslash \Gamma^{+}(I)}=p^{I} \sum_{S \subseteq \Gamma^{+}(I)} q_{S}$, as required.

Claim 5.18 (Simultaneous positivity of $q_{S}$ and $\breve{q}_{S}$ ). Assume that $p \in[0,1]^{n}$. Then

$$
\begin{array}{lll}
q_{I} \geq 0 \forall I \in \text { Ind } & \Longrightarrow & \breve{q}_{S} \geq q_{\emptyset} \forall S \subseteq[n] \\
\breve{q}_{S} \geq 0 \forall S \subseteq[n] \quad \Longrightarrow & q_{I} \geq p^{[n]} \cdot \breve{q}_{[n]} \forall I \in \text { Ind }
\end{array}
$$

Proof. (8) follows from Claim 5.14 (since $q_{Y}=0$ for $Y \notin$ Ind). To see (9), first note that $q_{I} \geq 0$ for all $I \in$ Ind, by Claim 5.16. Consequently, by Claim 5.14, $\breve{q}_{[n]}=\min _{S} \breve{q}_{S}$. Clearly, $p^{[n]}=\min _{I} p^{I}$. It follows from Claim 5.16 again that $q_{I}=p^{I} \cdot \breve{q}_{[n] \backslash \Gamma^{+}(I)} \geq p^{[n]} \cdot \breve{q}_{[n]}$.

Claim 5.19. The two characterizations of the Shearer region, (7a) and (7b), are equivalent.

Proof. By Claim 5.18, if $q_{\emptyset}>0$ and $q_{S} \geq 0 \forall S \subseteq[n]$, then $\breve{q}_{S}>0$ for all $S \subseteq[n]$. Conversely, if $\breve{q}_{S}>0$ for all $S \subseteq[n]$, then $q_{I} \geq p^{[n]} \breve{q}_{[n]}>0$ for all $I \in$ Ind.

Claim 5.20 (Monotonicity of $\breve{q}$, Scott-Sokal [35, Theorem 2.10]). Let $p \in[0,1]^{n}$.

$$
\breve{q}_{S}(p) \geq 0 \forall S \subseteq[n] \quad \Longrightarrow \quad \breve{q}_{S}\left(p^{\prime}\right) \geq \breve{q}_{S}(p) \quad \forall 0 \leq p^{\prime} \leq p, \forall S \subseteq[n] .
$$

Proof. First consider the case that $p$ and $p^{\prime}$ differ only in coordinate $i$. For any $S \subseteq[n]$, Claim 5.13 implies that $\frac{\partial}{\partial p_{i}} \breve{q}_{S}(p)=-\breve{q}_{S \backslash \Gamma^{+}(i)}(p)$ and $\frac{\partial^{2}}{\partial p_{i}^{2}} \breve{q}_{S}=0$. Thus,

$$
\breve{q}_{S}\left(p^{\prime}\right)=\breve{q}_{S}(p)+\left(p_{i}-p_{i}^{\prime}\right) \cdot \breve{q}_{S \backslash \Gamma^{+}(i)}(p) \geq \breve{q}_{S}(p) .
$$

The case that $p^{\prime}$ and $p$ differ in multiple coordinates is handled by induction.

Claim 5.21 (Log-submodularity of $\breve{q}_{S}$, Scott-Sokal [35, Corollary 2.27]). For any $p \in \mathcal{S}$ and $A, B \subseteq[n]$, we have $\breve{q}_{A} \cdot \breve{q}_{B} \geq \breve{q}_{A \cup B} \cdot \breve{q}_{A \cap B}$.

Proof. We claim that for any $a \in S \subseteq T$, we have

$$
\frac{\breve{q}_{S}}{\breve{q}_{S \backslash\{a\}}} \geq \frac{\breve{q}_{T}}{\breve{q}_{T \backslash\{a\}}}
$$

By induction, this implies that for any $R \subseteq S, \frac{\breve{q}_{S}}{\breve{q}_{S \backslash R}} \geq \frac{\breve{q}_{T}}{\breve{q}_{T \backslash R}}$. We obtain the claim above by setting $S=A$, $T=A \cup B$, and $R=A \backslash B$. 
We prove (10) again by induction, on $|T|$. For $|T|=1$, the statement is trivial. Let $|T|>1$. By Claim 5.13, we have

$$
\breve{q}_{S}=\breve{q}_{S \backslash\{a\}}-p_{a} \breve{q}_{S \backslash \Gamma^{+}(a)}
$$

and

$$
\breve{q}_{T}=\breve{q}_{T \backslash\{a\}}-p_{a} \breve{q}_{T \backslash \Gamma^{+}(a)} \cdot
$$

Let us denote $S \cap \Gamma^{+}(a)=\left\{a, s_{1}, \ldots, s_{k}\right\}$. We apply (10) to strict subsets of $S$ and $T$, to obtain

$$
\frac{\breve{q}_{S \backslash \Gamma^{+}(a)}}{\breve{q}_{S \backslash\{a\}}}=\prod_{i=1}^{k} \frac{\breve{q}_{S \backslash\left\{a, s_{1}, \ldots, s_{i-1}, s_{i}\right\}}}{\breve{q}_{S \backslash\left\{a, s_{1}, \ldots, s_{i-1}\right\}}} \leq \prod_{i=1}^{k} \frac{\breve{q}_{T \backslash\left\{a, s_{1}, \ldots, s_{i-1}, s_{i}\right\}}}{\breve{q}_{T \backslash\left\{a, s_{1}, \ldots, s_{i-1}\right\}}}=\frac{\breve{q}_{T \backslash\left(S \cap \Gamma^{+}(a)\right)}}{\breve{q}_{T \backslash\{a\}}} \leq \frac{\breve{q}_{T \backslash \Gamma^{+}(a)}}{\breve{q}_{T \backslash\{a\}}}
$$

where in the last step we used the monotonicity of $\breve{q}_{T}$ in $T$ (again from Claim 5.13). This implies (10):

$$
\frac{\breve{q}_{S}}{\breve{q}_{S \backslash\{a\}}}=1-p_{a} \frac{\breve{q}_{S \backslash \Gamma^{+}(a)}}{\breve{q}_{S \backslash\{a\}}} \geq 1-p_{a} \frac{\breve{q}_{T \backslash \Gamma^{+}(a)}}{\breve{q}_{T \backslash\{a\}}}=\frac{\breve{q}_{T}}{\breve{q}_{T \backslash\{a\}}} .
$$

Claim 5.22 (Log-submodularity of $q_{S}$ ). For any $p \in \mathcal{S}$ and $A, B \subseteq[n]$, we have $q_{A} \cdot q_{B} \geq q_{A \cup B} \cdot q_{A \cap B}$.

Proof. We can assume $A \cup B \in$ Ind; otherwise the right-hand side is zero. By Claim5.16, we have

$$
q_{A} \cdot q_{B}=p^{A} \breve{q}_{[n] \backslash \Gamma^{+}(A)} \cdot p^{B} \breve{q}_{[n] \backslash \Gamma^{+}(B)} \cdot
$$

By Claim 5.21,

$$
\breve{q}_{[n] \backslash \Gamma^{+}(A)} \cdot \breve{q}_{[n] \backslash \Gamma^{+}(B)} \geq \breve{q}_{[n] \backslash\left(\Gamma^{+}(A) \cup \Gamma^{+}(B)\right)} \cdot \breve{q}_{[n] \backslash\left(\Gamma^{+}(A) \cap \Gamma^{+}(B)\right)} \cdot
$$

Here we use the fact that $\Gamma^{+}(A) \cup \Gamma^{+}(B)=\Gamma^{+}(A \cup B)$, and $\Gamma^{+}(A) \cap \Gamma^{+}(B) \supseteq \Gamma^{+}(A \cap B)$. Therefore, by the monotonicity of $\breve{q}_{S}$,

$$
\breve{q}_{[n] \backslash \Gamma^{+}(A)} \cdot \breve{q}_{[n] \backslash \Gamma^{+}(B)} \geq \breve{q}_{[n] \backslash \Gamma^{+}(A \cup B)} \cdot \breve{q}_{[n] \backslash \Gamma^{+}(A \cap B)} \cdot
$$

Also, $p^{A} p^{B}=p^{A \cup B} p^{A \cap B}$. Using Claim 5.16 one more time, we obtain

$$
q_{A} \cdot q_{B} \geq p^{A \cup B} \breve{q}_{[n] \backslash \Gamma^{+}(A \cup B)} \cdot p^{A \cap B} \breve{q}_{[n] \backslash \Gamma^{+}(A \cap B)}=q_{A \cup B} \cdot q_{A \cap B} \cdot
$$

Claim 5.23. Suppose that $p \in \mathcal{S}$. For any set $S \subseteq[n]$,

$$
\sum_{J \subseteq S} \frac{q_{J}}{q_{\emptyset}} \leq \prod_{j \in S}\left(1+\frac{q_{\{j\}}}{q_{\emptyset}}\right)
$$

Proof. The proof is by induction on $S$, the case $|S| \leq 1$ being trivial. Fix any $s \in S$. Claim 5.22 implies that $q_{J+s} \cdot q_{\emptyset} \leq q_{\{s\}} \cdot q_{J}$ for any $J \subseteq S \backslash\{s\}$. Summing over $J$ yields

$$
\sum_{J \subseteq S \backslash\{s\}} \frac{q_{J+s}}{q_{\emptyset}} \leq \frac{q_{\{s\}}}{q_{\emptyset}} \sum_{J \subseteq S \backslash\{s\}} \frac{q_{J}}{q_{\emptyset}} .
$$

Adding $\sum_{J \subseteq S \backslash\{s\}} \frac{q_{J}}{q_{\emptyset}}$ to both sides yields

$$
\sum_{J \subseteq S} \frac{q_{J}}{q_{\emptyset}} \leq\left(1+\frac{q_{\{s\}}}{q_{\emptyset}}\right) \sum_{J \subseteq S \backslash\{s\}} \frac{q_{J}}{q_{\emptyset}} .
$$

The claim follows by induction. 
Claim 5.24. If $q_{\emptyset}>0$ then $\frac{q_{\{i\}}}{q_{\emptyset}}=\frac{\breve{q}_{[n] \backslash\{i\}}}{\breve{q}_{[n]}}-1$.

Proof. By Claim 5.14,

$$
1+\frac{q_{\{i\}}}{q_{\emptyset}}=\frac{q_{\emptyset}+q_{\{i\}}}{q_{\emptyset}}=\frac{\breve{q}_{[n] \backslash\{i\}}}{\breve{q}_{[n]}} .
$$

Claim 5.25 (Kolipaka-Szegedy [24, Theorem 5]). If $(1+\epsilon) p \in \mathcal{S}$ then $\frac{q_{\{i\}}}{q_{\emptyset}} \leq \frac{1}{\epsilon}$ for each $i \in[n]$.

Proof. Note that $\breve{q}_{[n] \backslash\{i\}}(p)$ does not depend on $p_{i}$, while $\breve{q}_{[n]}(p)$ is linear in $p_{i}$. Also, both quantities are equal at $p_{i}=0$ : we have $\breve{q}_{[n]}\left(p_{1}, \ldots, 0 \cdot p_{i}, \ldots, p_{n}\right)=\breve{q}_{[n] \backslash\{i\}}(p)$. Since $(1+\epsilon) p \in \mathcal{S}$, we know that $\breve{q}_{[n]}\left(p_{1}, \ldots,(1+\epsilon) p_{i}, \ldots, p_{n}\right) \geq 0$. By linearity, $\breve{q}_{[n]}(p) \geq \frac{\epsilon}{1+\epsilon} \breve{q}_{[n] \backslash\{i\}}(p)$. Claim 5.24 then implies that $\frac{q_{\{i\}}}{q_{\emptyset}} \leq \frac{1}{\epsilon}$.

\subsubsection{Connection to stable set sequences}

Kolipaka and Szegedy showed that stable set sequences relate to the independence polynomials $q_{S}$. The following is the crucial upper-bound for stable set sequences when Shearer's criterion holds. In fact, this result is subsumed by Lemma 5.27 but we present the upper bound first, with a shorter proof.

Lemma 5.26 (Kolipaka-Szegedy [24]). If $q_{S} \geq 0$ for all $S \subseteq[n]$ and $q_{\emptyset}>0$, then

$$
\sum_{\mathcal{I} \in \operatorname{Stab}_{\ell}(J)} p_{\mathcal{I}} \leq \frac{q_{J}}{q_{\emptyset}} \quad \forall J \in \operatorname{lnd}, \forall \ell \geq 1 .
$$

Proof. We proceed by induction: for $\ell=1$, there is only one such stable set sequence $\mathcal{I}=(J)$. By Lemma 5.17, we have $q_{J}=p^{J} \sum_{S \subseteq \Gamma^{+}(J)} q_{S} \geq p^{J} q_{\emptyset}$. (Recall that $q_{S} \geq 0$ for all $S \subseteq[n]$.) Hence, $p_{(J)}=p^{J} \leq q_{J} / q_{\emptyset}$.

The inductive step: every stable set sequence starting with $J$ has the form $\mathcal{I}=\left(J, J^{\prime}, \ldots\right)$ where $J^{\prime} \subseteq \Gamma^{+}(J)$. Therefore,

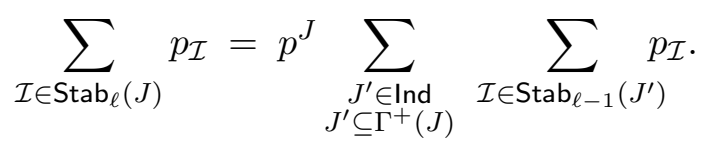

By the inductive hypothesis, $\sum_{\mathcal{I} \in \operatorname{Stab}_{\ell-1}\left(J^{\prime}\right)} p_{\mathcal{I}} \leq q_{J^{\prime}} / q_{\emptyset}$. Also, recall that $q_{J^{\prime}}=0$ if $J^{\prime} \notin$ Ind. Therefore,

$$
\sum_{\mathcal{I} \in \operatorname{Stab}_{\ell}(J)} p_{\mathcal{I}} \leq p^{J} \sum_{J^{\prime} \subseteq \Gamma^{+}(J)} \frac{q_{J^{\prime}}}{q_{\emptyset}}=\frac{q_{J}}{q_{\emptyset}}
$$

using Lemma 5.17 to obtain the last equality.

The inequality in Lemma 5.26 actually becomes an equality as $\ell \rightarrow \infty$, as shown in Lemma 5.27 This stronger result is used only tangentially in Section 5.7.2, but we provide a detailed proof in order to clarify the arguments of Kolipaka and Szegedy [24].

Lemma 5.27 (Kolipaka-Szegedy [24, Theorem 14]). For a dependency graph $G$ and $p_{1}, \ldots, p_{n} \in(0,1)$, the following statements are equivalent:

1. $q_{\emptyset}>0$ and $q_{S} \geq 0$ for all $S \subseteq[n]$. 
2. for all $J \in \operatorname{Ind}, q_{J}>0$ and $\sum_{\mathcal{I} \in \operatorname{Prop}(J)} p_{\mathcal{I}}=q_{J} / q_{\emptyset}$.

3. $\sum_{\mathcal{I} \in \operatorname{Prop}(J)} p_{\mathcal{I}}$ is finite for each $J \in$ Ind.

Proof. First, note that $\operatorname{Prop}(J)=\bigcup_{t=1}^{\infty} \operatorname{Prop}_{t}(J)$, and $\bigcup_{t=1}^{\ell} \operatorname{Prop}_{t}(J)$ can be identified with $\operatorname{Stab}_{\ell}(J)$, since each proper sequence $\mathcal{I}$ of length at most $\ell$ can be padded with empty sets to obtain a sequence in $\operatorname{Stab}_{\ell}(J)$ (and $p_{\mathcal{I}}$ does not change). Therefore, $\sum_{\mathcal{I} \in \operatorname{Prop}(J)} p_{\mathcal{I}}=\lim _{\ell \rightarrow \infty} \sum_{\mathcal{I} \in \operatorname{Stab}_{\ell}(J)} p_{\mathcal{I}}$. This is a non-decreasing sequence; the limit exists but could be infinite. Let us denote $w_{J}^{(\ell)}=\sum_{\mathcal{I} \in \operatorname{Stab}_{\ell}(J)} p_{\mathcal{I}}$ and $w_{J}^{*}=\lim _{\ell \rightarrow \infty} w_{J}^{(\ell)}=\sum_{\mathcal{I} \in \operatorname{Prop}(J)} p_{\mathcal{I}}$. Let us define $M$ to be the following linear operator on $\mathbb{R}^{\text {Ind. }}$ :

$$
(M x)_{I}=p^{I} \sum_{\substack{J \in \operatorname{lnd} \\ J \subseteq \Gamma^{+}(I)}} x_{J}
$$

Using this notation, the identity (11) can written compactly as $w^{(\ell)}=M w^{(\ell-1)}$. Inductively, $w^{(\ell)}=$ $M^{\ell-1} w^{(1)}$, and $w^{*}=\lim _{\ell \rightarrow \infty} M^{\ell} w^{(1)}$.

$1 \Rightarrow 2$ : Assume now that $q_{S} \geq 0$ for all $S \subseteq[n]$ and $q_{\emptyset}>0$. Lemma 5.26 proves that this implies $w_{J}^{*}=\sum_{\mathcal{I} \in \operatorname{Prop}(J)} p_{\mathcal{I}}=\lim _{\ell \rightarrow \infty} \sum_{\mathcal{I} \in \operatorname{Stab}_{\ell}(J)} p_{\mathcal{I}} \leq q_{J} / q_{\emptyset}$. Clearly $\sum_{\mathcal{I} \in \operatorname{Prop}(J)} p_{\mathcal{I}}>0$, so this also implies that $q_{J}>0$ for all $J \in$ Ind.

Note that $w^{(1)}$ is the column of $M$ corresponding to $J=\emptyset: M_{I, \emptyset}=p^{I}$ for each $I \in \operatorname{Ind}$. Therefore, we can write $w^{(1)}=M w^{(0)}$, where $w^{(0)}=e_{\emptyset}$ is the canonical basis vector in $\mathbb{R}^{\text {Ind }}$ corresponding to $\emptyset$. We have $w^{*}=\lim _{\ell \rightarrow \infty} M^{\ell} w^{(1)}=\lim _{\ell \rightarrow \infty} M^{\ell} w^{(0)}$. We may subtract these two limits since we have shown that every $w_{J}^{*}$ is finite, obtaining $\lim _{\ell \rightarrow \infty} M^{\ell}\left(w^{(1)}-w^{(0)}\right)=0$. We note that $w^{(1)}-w^{(0)}$ has strictly positive coordinates for $I \neq \emptyset$, and 0 for $I=\emptyset$.

By Lemma 5.17, we have $M q=q$ for the vector $q \in \mathbb{R}^{\text {Ind }}$ with coordinates $q_{I}$. Consider $\frac{1}{q_{\emptyset}} q-w^{(0)}$, a nonnegative vector with 0 in the coordinate corresponding to $\emptyset$. We can choose $\beta>0$ large enough so that coordinate-wise, $0 \leq \frac{1}{q_{\emptyset}} q-w^{(0)} \leq \beta\left(w^{(1)}-w^{(0)}\right)$. From this we derive that

$$
0 \leq \frac{1}{q_{\emptyset}} q-w^{*}=\lim _{\ell \rightarrow \infty} M^{\ell}\left(\frac{1}{q_{\emptyset}} q-w^{(0)}\right) \leq \beta \lim _{\ell \rightarrow \infty} M^{\ell}\left(w^{(1)}-w^{(0)}\right)=0,
$$

so equality holds throughout. Recalling the definition of $w_{J}^{*}$, we conclude that $\sum_{\mathcal{I} \in \operatorname{Prop}(J)} p_{\mathcal{I}}=w_{J}^{*}=\frac{1}{q_{\emptyset}} q_{J}$.

$2 \Rightarrow 3$ : Trivial.

$\mathbf{3} \Rightarrow \mathbf{1}$ : Let $p \in(0,1)^{n}$ be the vector $\left(p_{1}, \ldots, p_{n}\right)$. We can assume that $\min _{S} \breve{q}_{S}(p) \leq 0$, otherwise we are done by Claim 5.18 Let us consider the values of $\breve{q}_{S}$ on the line $\{\lambda p: \lambda \in[0,1]\}$. Define $\lambda^{*}=$ $\inf \left\{\lambda \in(0,1]: \min _{S} \breve{q}_{S}(\lambda p) \leq 0\right\}$. We observe that $\min _{S} \breve{q}_{S}(\lambda p)>0$ for $0<\lambda<1 / n$, which can be verified directly by considering the alternating sum defining $\breve{q}_{S}$. (Intuitively, Shearer's Lemma holds in this region just by the union bound.) Therefore, we have $\lambda^{*}>0$. Furthermore continuity also implies $\min _{S} \breve{q}_{S}\left(\lambda^{*} p\right)=0$, so Claim 5.18 yields $q_{\emptyset}\left(\lambda^{*} p\right)=\breve{q}_{[n]}\left(\lambda^{*} p\right)=0$. For $\lambda \in\left[0, \lambda^{*}\right)$ we have $\min _{S} \breve{q}_{S}(\lambda p)>$ 0 , so by Claim 5.18 we also have $\min _{I \in \text { Ind }} q_{I}(\lambda p)>0$. This shows that the condition 1 holds at the point $\lambda p$, for $\lambda \in\left[0, \lambda^{*}\right)$, so we may use the implication $1 \Rightarrow \mathbf{2}: \sum_{\mathcal{I} \in \operatorname{Prop}(J)}(\lambda p)_{\mathcal{I}}=q_{J}(\lambda p) / q_{\emptyset}(\lambda p)$. Let $J \in$ Ind be such that $q_{J}\left(\lambda^{*} p\right)>0$; such a $J$ must exist by Claim 5.15. By the monotonicity of $p_{\mathcal{I}}=\prod_{I \in \mathcal{I}} p^{I}$ in the variables $p_{1}, \ldots, p_{n}$, we have

$$
\sum_{\mathcal{I} \in \operatorname{Prop}(J)} p_{\mathcal{I}} \geq \sum_{\mathcal{I} \in \operatorname{Prop}(J)}\left(\lambda^{*} p\right)_{\mathcal{I}} \geq \liminf _{\lambda \rightarrow \lambda^{*}-} \sum_{\mathcal{I} \in \operatorname{Prop}(J)}(\lambda p)_{\mathcal{I}}=\liminf _{\lambda \rightarrow \lambda^{*}-} \frac{q_{J}(\lambda p)}{q_{\emptyset}(\lambda p)}=\infty,
$$

as $q_{J}\left(\lambda^{*} p\right)>0$ but $q_{\emptyset}\left(\lambda^{*} p\right)=0$. This contradicts the assumption 3 that $\sum_{\mathcal{I} \in \operatorname{Prop}(J)} p_{\mathcal{I}}$ is finite. 
From Claim 5.15, we obtain immediately the following.

Corollary 5.28. If $q_{S} \geq 0$ for all $S \subseteq[n]$ and $q_{\emptyset}>0$,

$$
\sum_{\mathcal{I} \in \operatorname{Prop}} p_{\mathcal{I}}=\frac{1}{q_{\emptyset}}
$$

Remark. An equivalent statement using the language of "traces" appears in the recent manuscript of Knuth [23. Page 86, Theorem F], together with a short proof using generating functions. Furthermore, using Claim 5.14, we may derive

$$
\sum_{J \subseteq A} \sum_{\mathcal{I} \in \operatorname{Prop}(J)} p_{\mathcal{I}}=\sum_{J \subseteq A} \frac{q_{J}}{q_{\emptyset}}=\frac{\breve{q}_{[n] \backslash A}}{\breve{q}_{[n]}},
$$

for any $A \subseteq[n]$. This statement, in the language of traces, also appears in Knuth's draft [23, Page 87, Equation (144)].

Summary at this point. By Lemma 5.6 and Corollary 5.28, MaximalSetResample produces a state in $\bigcap_{i=1}^{n} \overline{E_{i}}$ after at most $1 / q_{\emptyset}$ iterations in expectation. However, this should not be viewed as a statement of efficiency. Shearer's Lemma proves that $\operatorname{Pr}_{\mu}\left[\bigcap_{i=1}^{n} \overline{E_{i}}\right] \geq q_{\emptyset}$ so, in expectation, $1 / q_{\emptyset}$ independent samples from $\mu$ would also suffice to find a state in $\bigcap_{i=1}^{n} \overline{E_{i}}$.

Section 5.4 improves this analysis by assuming that Shearer's criterion holds with some slack, analogous to the result in Section 5.2. Section 5.5 then removes the need for that assumption — it argues that Shearer's criterion always holds with some slack, and provides quantitative bounds on that slack.

\subsection{Shearer's criterion with slack}

In this section we consider scenarios in which Shearer's criterion holds with a certain amount of slack. To make this formal, we will consider another vector $p^{\prime}$ of probabilities with $p \leq p^{\prime} \in \mathcal{S}$. For notational convenience, we will let $q_{S}^{\prime}$ denote the value $q_{S}\left(p^{\prime}\right)$ and let $q_{S}$ denote $q_{S}(p)$ as before. Let us assume that Shearer's criterion holds with some slack in the following natural sense.

Definition 5.29. We say that $p \in(0,1)^{n}$ satisfies Shearer's criterion with coefficients $q_{S}^{\prime}$ at a slack of $\epsilon$, if $p^{\prime}=(1+\epsilon) p$ is still in the Shearer region $\mathcal{S}$ and $q_{S}^{\prime}=q_{S}\left(p^{\prime}\right)$.

Theorem 5.30. Recall that $p_{i}=\operatorname{Pr}_{\mu}\left[E_{i}\right]$. If the $p_{i}$ satisfy Shearer's criterion with coefficient $q_{\emptyset}^{\prime}$ at a slack of $\epsilon \in(0,1)$, then the probability that MaximalSetResample resamples more than $\frac{2}{\epsilon}\left(\ln \frac{1}{q_{\emptyset}^{\prime}}+t\right)$ events is at most $e^{-t}$.

Proof. By Lemma 5.6 the probability that MaximalSetResample resamples more than $s$ events is at most $\sum_{\mathcal{I} \in \operatorname{Prop}: \sigma(\mathcal{I})=\lceil s\rceil} p_{\mathcal{I}}$. By the slack assumption, we have

$$
\operatorname{Pr}[\text { resample more than } s \text { events }] \leq \sum_{\substack{\mathcal{I} \in \operatorname{Prop} \\ \sigma(\mathcal{I})=\lceil s\rceil}} p_{\mathcal{I}} \leq(1+\epsilon)^{-s} \sum_{\substack{\mathcal{I} \in \text { Prop } \\ \sigma(\mathcal{I})=\lceil s\rceil}} p_{\mathcal{I}}^{\prime}
$$

since we have $p_{i}^{\prime}=(1+\epsilon) p_{i}$ for each event appearing in a sequence $\mathcal{I}$. The hypothesis is that the probabilities $p_{i}^{\prime}$ satisfy Shearer's criterion with a bound of $q_{\emptyset}^{\prime}$. Consequently, Corollary 5.28 implies that $\sum_{\mathcal{I} \in \operatorname{Prop}: \sigma(\mathcal{I})=\lceil s\rceil} p_{\mathcal{I}}^{\prime} \leq \sum_{\mathcal{I} \in \text { Prop }} p_{\mathcal{I}}^{\prime} \leq 1 / q_{\emptyset}^{\prime}$. Thus, for $s=\frac{2}{\epsilon}\left(\ln \frac{1}{q_{\emptyset}^{\prime}}+t\right)$ we obtain

$$
\operatorname{Pr}[\text { resample more than } s \text { events }] \leq(1+\epsilon)^{-s} \frac{1}{q_{\emptyset}^{\prime}} \leq e^{-s \epsilon / 2} \frac{1}{q_{\emptyset}^{\prime}} \leq e^{-\left(\ln \left(1 / q_{\emptyset}^{\prime}\right)+t\right)} \frac{1}{q_{\emptyset}^{\prime}}=e^{-t} .
$$


In other words, the probability that MaximalSetResample requires more than $\frac{2}{\epsilon} \ln \left(1 / q_{\emptyset}^{\prime}\right)$ resamplings decays exponentially fast; in particular the expected number of resampled events is $O\left(\frac{1}{\epsilon} \ln \left(1 / q_{\emptyset}^{\prime}\right)\right)$. This appears significantly better than the trivial bound of $1 / q_{\emptyset}$; still, it is not clear whether this bound can be considered "polynomial". In the following, we show that this leads in fact to efficient bounds, comparable to the best known bounds in the variable model.

Corollary 5.31. If the $p_{i}$ satisfy Shearer's criterion with coefficients $q_{S}^{\prime}$ at a slack of $\epsilon \in(0,1)$, then the probability that MaximalSetResample resamples more than

$$
\frac{2}{\epsilon}\left(\sum_{j=1}^{n} \ln \left(1+\frac{q_{\{j\}}^{\prime}}{q_{\emptyset}^{\prime}}\right)+t\right)
$$

events is at most $e^{-t}$.

Proof. By Claim 5.15 and Claim 5.23, we have

$$
\ln \frac{1}{q_{\emptyset}^{\prime}}=\ln \sum_{J \subseteq[n]} \frac{q_{J}^{\prime}}{q_{\emptyset}^{\prime}} \leq \sum_{j=1}^{n} \ln \left(1+\frac{q_{\{j\}}^{\prime}}{q_{\emptyset}^{\prime}}\right) .
$$

The result follows from Theorem 5.30 .

Next, we provide a simplified bound that depends only on the amount of slack and the number of events. This is analogous to a bound of $O(n / \epsilon)$ given by Kolipaka-Szegedy [24] in the variable model.

Theorem 5.32. If $p_{1}, \ldots, p_{n}$ satisfy Shearer's criterion at a slack of $\epsilon \in(0,1)$, then the expected number of events resampled by MaximalSetResample is $O\left(\frac{n}{\epsilon} \log \frac{1}{\epsilon}\right)$.

Proof. Let $p^{\prime}=(1+\epsilon / 2) p$. By assumption, $(1+\epsilon / 3) p^{\prime} \leq(1+\epsilon) p \in \mathcal{S}$. Therefore, $p^{\prime}$ still has $\epsilon / 3$ slack so by Claim 5.25, the coefficients $q_{S}^{\prime}=q_{S}\left(p^{\prime}\right)$ satisfy $\frac{q_{\{i\}}^{\prime}}{q_{\emptyset}^{\prime}} \leq \frac{3}{\epsilon}$. The point $p$ satisfies Shearer's criterion with coefficients $q_{S}^{\prime}$ at a slack of $\epsilon / 2$, so by Corollary 5.31, the probability that we resample more than $\frac{4}{\epsilon}\left(n \ln \left(1+\frac{3}{\epsilon}\right)+t\right)$ events is at most $e^{-t}$. In expectation, we resample $O\left(\frac{n}{\epsilon} \log \frac{1}{\epsilon}\right)$ events as claimed.

\subsection{Quantification of slack in Shearer's criterion}

In the previous section, we proved a bound on the number of resamplings in the MaximalSetResample algorithm, provided that Shearer's criterion is satisfied with a certain slack. In fact, from Definition 5.11 one can observe that the Shearer region is an open set and therefore there is always a certain amount of slack. However, how large a slack we can assume is not a priori clear. In particular, one can compare with Kolipaka-Szegedy [24] where a bound is proved on the expected number of events one has to resample in the variable model: If Shearer's criterion is satisfied with coefficients $q_{S}$, then the expected number of resamplings is at most $\sum_{i=1}^{n} q_{\{i\}} / q_{\emptyset}[24]$. In this section, we prove that anywhere in the Shearer region, there is an amount of slack inversely proportional to this quantity, which leads to a bound similar to that of Kolipaka and Szegedy [24].

Lemma 5.33. Let $\left(p_{1}, \ldots, p_{n}\right) \in(0,1)^{n}$ be a point in the Shearer region. Let $\epsilon=q_{\emptyset} /\left(2 \sum_{i=1}^{n} q_{\{i\}}\right)$ and $p_{i}^{\prime}=(1+\epsilon) p_{i}$. Then $\left(p_{1}^{\prime}, \ldots, p_{n}^{\prime}\right)$ is also in the Shearer region, and $q_{\emptyset}\left(p^{\prime}\right) \geq \frac{1}{2} q_{\emptyset}(p)$. 
Before proving the lemma, let us consider the partial derivatives of the $\breve{q}_{S}$ polynomials.

Claim 5.34. For any $i \in S$,

$$
\frac{\partial \breve{q}_{S}}{\partial p_{i}}=-\breve{q}_{S \backslash \Gamma^{+}(i)}
$$

and for any $j \in S \backslash \Gamma^{+}(i)$,

$$
\frac{\partial^{2} \breve{q}_{S}}{\partial p_{i} \partial p_{j}}=\breve{q}_{S \backslash \Gamma^{+}(i) \backslash \Gamma^{+}(j)} .
$$

For other choices of $i, j$, the partial derivatives are 0. In particular, for any point in the Shearer region, $\frac{\partial \breve{q}_{S}}{\partial p_{i}} \leq 0$ and $\frac{\partial^{2} \breve{q}_{S}}{\partial p_{i} \partial p_{j}} \geq 0$.

Due to Claim 5.34 we may say that $\breve{q}_{S}\left(p_{1}, \ldots, p_{n}\right)$ is "continuous supermodular" in the Shearer region. Proof. For any $i \in S$, we have $\breve{q}_{S}=\breve{q}_{S \backslash\{i\}}-p_{i} \breve{q}_{S \backslash \Gamma^{+}(i)}$ by Claim 5.13 The polynomials $\breve{q}_{S \backslash\{i\}}$ and $\breve{q}_{S \backslash \Gamma^{+}(i)}$ do not depend on $p_{i}$ and hence $\frac{\partial \breve{q}_{S}}{\partial p_{i}}$ is equal to $-\breve{q}_{S \backslash \Gamma^{+}(i)}$. Repeating this argument one more time for $j \in S \backslash \Gamma^{+}(i)$, we get $\frac{\partial \breve{q}_{S}}{\partial p_{i}}=-\breve{q}_{S \backslash \Gamma^{+}(i)}=-\breve{q}_{S \backslash \Gamma^{+}(i) \backslash\{j\}}+p_{j} \breve{q}_{S \backslash \Gamma^{+}(i) \backslash \Gamma^{+}(j)}$. Again, $\breve{q}_{S \backslash \Gamma^{+}(i) \backslash\{j\}}$ and $\breve{q}_{S \backslash \Gamma^{+}(i) \backslash \Gamma^{+}(j)}$ do not depend on $p_{j}$ and hence $\frac{\partial^{2} \breve{q}_{S}}{\partial p_{i} \partial p_{j}}=\breve{q}_{S \backslash \Gamma^{+}(i) \backslash \Gamma^{+}(j)}$.

Clearly, we have $\frac{\partial \breve{q}_{S}}{\partial p_{i}}=0$ unless $i \in S$, and $\frac{\partial^{2} \breve{q}_{S}}{\partial p_{i} \partial p_{j}}=0$ unless $i \in S$ and $j \in S \backslash \Gamma^{+}(i)$. Since all the coefficients $\breve{q}_{S}$ are positive in the Shearer region, we have $\frac{\partial \breve{q}_{S}}{\partial p_{i}} \leq 0$ and $\frac{\partial^{2} \breve{q}_{S}}{\partial p_{i} \partial p_{j}} \geq 0$ for all $i, j$.

Now we can prove Lemma 5.33

Proof. Consider the line segment from $p=\left(p_{1}, \ldots, p_{n}\right)$ to $p^{\prime}=\left(p_{1}^{\prime}, \ldots, p_{n}^{\prime}\right)$ where $p_{i}^{\prime}=(1+\epsilon) p_{i}$, $\epsilon=\frac{q_{\emptyset}}{2 \sum_{i=1}^{n} q_{\{i\}}}$. Note that $p_{i}^{\prime} \leq\left(1+\frac{q_{\emptyset}}{q_{\{i\}}}\right) p_{i}=\frac{q_{\{i\}}+q_{\emptyset}}{q_{\{i\}}} p_{i}=\frac{\breve{q}_{[n] \backslash\{i\}}}{p_{i} \tilde{q}_{[n] \backslash \Gamma}+(i)} p_{i} \leq 1$ by Claim 5.14, Claim 5.16 and Claim 5.20, Let us define

$$
Q_{\emptyset}(\lambda)=q_{\emptyset}\left((1+\lambda) p_{1}, \ldots,(1+\lambda) p_{n}\right) .
$$

By the chain rule and Claim 5.34, we have

$$
\left.\frac{d Q_{\emptyset}}{d \lambda}\right|_{\lambda=0}=\sum_{i=1}^{n} p_{i} \frac{\partial q_{\emptyset}}{\partial p_{i}}=-\sum_{i=1}^{n} p_{i} \breve{q}_{[n] \backslash \Gamma^{+}(i)}=-\sum_{i=1}^{n} q_{\{i\}}
$$

where we used Claim 5.16 in the last equality. Assuming that $(1+\lambda) p=\left((1+\lambda) p_{1}, \ldots,(1+\lambda) p_{n}\right)$ is in the Shearer region, we also have by Claim 5.34

$$
\frac{d^{2} Q_{\emptyset}}{d \lambda^{2}}=\sum_{i, j=1}^{n} \frac{\partial^{2} q_{\emptyset}}{\partial p_{i} \partial p_{j}} p_{i} p_{j} \geq 0
$$

That is, $Q_{\emptyset}(\lambda)$ is a convex function for $\lambda \geq 0$ as long as $(1+\lambda) p$ is in the Shearer region. Our goal is to prove that this indeed happens for $\lambda \in[0, \epsilon]$.

Assume for the sake of contradiction that $(1+\lambda) p$ is not in the Shearer region for some $\lambda \in[0, \epsilon]$, and let $\lambda^{*}$ be the minimum such value (which exists since the complement of the Shearer region is closed). By Claim 5.18, anywhere in the Shearer region, $q_{\emptyset}=\breve{q}_{[n]}$ is the minimum of the $\breve{q}_{S}$ coefficients; hence by continuity it must be the case that $\breve{q}_{[n]}\left(\left(1+\lambda^{*}\right) p\right)$ is the minimum coefficient among $\breve{q}_{S}\left(\left(1+\lambda^{*}\right) p\right)$ for all 
$S \subseteq[n]$, and $Q_{\emptyset}\left(\lambda^{*}\right)=\breve{q}_{[n]}\left(\left(1+\lambda^{*}\right) p\right) \leq 0$. On the other hand, by the minimality of $\lambda^{*}, Q_{\emptyset}(\lambda)$ is positive and convex on $\left[0, \lambda^{*}\right)$ and therefore

$$
Q_{\emptyset}\left(\lambda^{*}\right) \geq Q_{\emptyset}(0)+\left.\lambda^{*} \frac{d Q_{\emptyset}}{d \lambda}\right|_{\lambda=0}=q_{\emptyset}-\lambda^{*} \sum_{i=1}^{n} q_{\{i\}} \geq q_{\emptyset}-\epsilon \sum_{i=1}^{n} q_{\{i\}}=\frac{1}{2} q_{\emptyset}>0,
$$

which is a contradiction. Therefore, $Q_{\emptyset}(\lambda)$ is positive and convex for all $\lambda \in[0, \epsilon]$. By the same computation as above, $Q_{\emptyset}(\epsilon) \geq \frac{1}{2} q_{\emptyset}$.

This implies our main algorithmic result under Shearer's criterion.

Theorem 5.35. Let $E_{1}, \ldots, E_{n}$ be events and let $p_{i}=\operatorname{Pr}_{\mu}\left[E_{i}\right]$. Suppose that the three subroutines described in Section 1.1.1 exist. If $p \in \mathcal{S}$ then the probability that MaximalSetResample resamples more than $4 \sum_{i=1}^{n} \frac{q_{\{i\}}}{q_{\emptyset}}\left(\sum_{j=1}^{n} \ln \left(1+\frac{q_{\{j\}}}{q_{\emptyset}}\right)+1+t\right)$ events is at most $e^{-t}$.

We note that the corresponding result in the variable model [24] was that the expected number of resamplings is at most $\sum_{i=1}^{n} \frac{q_{\{i\}}}{q \emptyset}$. Here, we obtain a bound which is at most quadratic in this quantity.

Proof. Directly from Theorem 5.30 and Lemma 5.33. Given $p$ in the Shearer region, Lemma 5.33 implies that $p$ in fact satisfies Shearer's criterion with a bound of $q_{\emptyset}^{\prime} \geq \frac{q_{\emptyset}}{2}$ at a slack of $\epsilon=\frac{q_{\emptyset}}{2} / \sum_{i=1}^{n} q_{\{i\}}$. By Theorem 5.30, the probability that MaximalSetResample resamples more than $s$ events is at most $e^{-t}$, where

$$
s=\frac{2}{\epsilon}\left(\ln \frac{1}{q_{\emptyset}^{\prime}}+t\right) \leq \frac{4}{q_{\emptyset}} \sum_{i=1}^{n} q_{\{i\}}\left(\ln \frac{1}{q_{\emptyset}}+1+t\right) .
$$

Using Claim 5.23, we can replace $\ln \frac{1}{q_{\emptyset}}$ by $\sum_{j=1}^{n} \ln \left(1+\frac{q_{\{j\}}}{q_{\emptyset}}\right)$.

\subsection{The General LLL criterion, without slack}

Shearer's Lemma (Lemma 5.12) is a strengthening of the original Lovász Local Lemma (Theorem 1.1): if $p_{1}, \ldots, p_{n}$ satisfy (GLL) then they must also satisfy Shearer's criterion $p \in \mathcal{S}$. Nevertheless, there does not seem to be a direct proof of this fact in the literature. Shearer [36] indirectly proves this fact by showing that, when $p \notin \mathcal{S}$ it is possible that $\operatorname{Pr}\left[\bigcap_{i=1}^{n} \overline{E_{i}}\right]=0$, so the contrapositive of Theorem 1.1 implies that (GLL) cannot hold. Scott and Sokal prove this fact using analytic properties of the partition function [35, Corollary 5.3]. In this section we establish this fact by an elementary, self-contained proof.

We then establish Theorem 1.2, our algorithmic form of Theorem 1.1 in the general framework of resampling oracles. Unlike the simpler analysis of Section 5.2, the analysis of this section does not assume any slack in the GLL criterion.

Lemma 5.36. Suppose that $p$ satisfies (GLL). Then, for every $S \subseteq[n]$ and $a \in S$, we have

$$
\frac{\breve{q}_{S}}{\breve{q}_{S \backslash\{a\}}} \geq 1-x_{a} \text {. }
$$

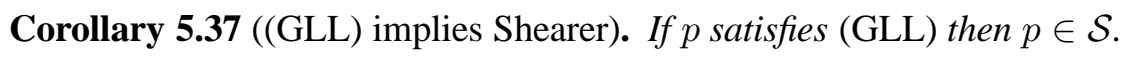

Proof. For any $S \subseteq[n]$, write it as $S=\left\{s_{1}, \ldots, s_{k}\right\}$. Induction yields

$$
\frac{\breve{q}_{S}}{\breve{q}_{\emptyset}}=\prod_{i=1}^{k} \frac{\breve{q}_{\left\{s_{1}, \ldots, s_{i}\right\}}}{\breve{q}_{\left\{s_{1}, \ldots, s_{i-1}\right\}}} \geq \prod_{a \in S}\left(1-x_{a}\right)>0 .
$$

The claim follows since $\breve{q}_{\emptyset}=1$. 
Corollary 5.38. If $p$ satisfies (GLL) then $\frac{q_{\{a\}}}{q_{\emptyset}} \leq \frac{x_{a}}{1-x_{a}}$.

Proof. Lemma 5.36 yields $\frac{\breve{q}_{[n]-a}}{\breve{q}_{[n]}} \leq \frac{1}{1-x_{a}}$, so the result follows from Claim 5.25,

Proof (of Lemma 5.36). We proceed by induction on $|S|$. The base case, $S=\emptyset$, is trivial: there is no $a \in S$ to choose. Consider $S \neq \emptyset$ and an element $a \in S$. By Claim 5.13, we have $\breve{q}_{S}=\breve{q}_{S \backslash\{a\}}-p_{a} \breve{q}_{S \backslash \Gamma^{+}(a)}$. By the inductive hypothesis applied iteratively to the elements of $(S \backslash\{a\}) \backslash\left(S \backslash \Gamma^{+}(a)\right)=\Gamma(a) \cap S$, we have

$$
\breve{q}_{S \backslash\{a\}} \geq \breve{q}_{S \backslash \Gamma^{+}(a)} \prod_{i \in \Gamma(a) \cap S}\left(1-x_{i}\right) .
$$

Therefore, we can write

$$
\breve{q}_{S}=\breve{q}_{S \backslash\{a\}}-p_{a} \breve{q}_{S \backslash \Gamma^{+}(a)} \geq \breve{q}_{S \backslash\{a\}}\left(1-\frac{p_{a}}{\prod_{i \in \Gamma(a) \cap S}\left(1-x_{i}\right)}\right) .
$$

By the claim's hypothesis, $p_{a} \leq x_{a} \prod_{i \in \Gamma(a)}\left(1-x_{i}\right) \leq x_{a} \prod_{i \in \Gamma(a) \cap S}\left(1-x_{i}\right)$, so we conclude that $\breve{q}_{S} \geq$ $\left(1-x_{a}\right) \breve{q}_{S \backslash\{a\}}$.

These results, together with our analysis of Shearer's criterion with slack (Corollary 5.31), immediately provide an analysis under the assumption that (GLL) holds with slack, similar to Theorem 5.8 However, this connection to Shearer's criterion allows us to prove more.

We show that our algorithm is in fact efficient even when the (GLL criterion is tight. This might be surprising in light of Corollary 5.28, which does not use any slack and gives an exponential bound of $\frac{1}{q_{\emptyset}}=\frac{1}{\breve{q}_{[n]}} \leq \prod_{i=1}^{n} \frac{1}{1-x_{i}}$. The reason why we can prove a stronger bound is that Shearer's criterion is never tight: as we argued already, it defines an open set, and Section 5.5 derives a quantitative bound on the slack that is always available under Shearer's criterion.

Theorem 5.39. Let $E_{1}, \ldots, E_{n}$ be events and let $p_{i}=\operatorname{Pr}_{\mu}\left[E_{i}\right]$. Suppose that the three subroutines described in Section 1.1.1 exist. If p satisfies (GLD) then the probability that MaximalSetResample resamples more than $4 \sum_{i=1}^{n} \frac{x_{i}}{1-x_{i}}\left(\sum_{j=1}^{n} \ln \frac{1}{1-x_{j}}+1+t\right)$ events is at most $e^{-t}$.

If (GLD) is satisfied with a slack of $\epsilon \in(0,1)$, i.e., $(1+\epsilon) p_{i} \leq x_{i} \prod_{j \in \Gamma(i)}\left(1-x_{j}\right)$, then with probability at least $1-e^{-t}$, MaximalSetResample resamples no more than $\frac{2}{\epsilon}\left(\sum_{j=1}^{n} \ln \frac{1}{1-x_{j}}+t\right)$ events.

Proof. The first part follows directly from Theorem 5.35, since Corollary 5.37 shows that $p \in \mathcal{S}$ and Corollary 5.38 shows that $\frac{q_{\{i\}}}{q_{\emptyset}} \leq \frac{x_{i}}{1-x_{i}}$. The second part follows from Corollary 5.31, using again that $\frac{q_{\{i\}}}{q_{\emptyset}} \leq \frac{x_{i}}{1-x_{i}}$.

Theorem 1.2 follows immediately from Theorem 5.39

\subsection{The cluster expansion criterion}

Recall that Section 1.4 introduced the cluster expansion criterion, which often gives improved quantitative bounds compared to the General LLL (such as the applications discussed in Section 44). For convenience, let us restate the cluster expansion criterion here. Given parameters $y_{1}, \ldots, y_{n}$, define the notation

$$
Y_{S}=\sum_{\substack{I \subseteq S \\ I \in \operatorname{lnd}}} y^{I} \quad \forall S \subseteq[n] .
$$


The cluster expansion criterion for a vector $p \in[0,1]^{n}$, with respect to a graph $G$, is

$$
\exists y_{1}, \ldots, y_{n}>0 \quad \text { such that } \quad p_{i} \leq y_{i} / Y_{\Gamma^{+}(i)} .
$$

This criterion was introduced in the following non-constructive form of the LLL.

Theorem 5.40 (Bissacot et al. [8]). Let $E_{1}, \ldots, E_{n}$ be events with a (lopsi-)dependency graph $G$, and let $p_{i}=\operatorname{Pr}_{\mu}\left[E_{i}\right]$. If $p$ and $G$ satisfy (CLL) then $\operatorname{Pr}_{\mu}\left[\bigcap_{i=1}^{n} \overline{E_{i}}\right]>0$.

To see that this strengthens the original LLL (Theorem 1.1), one may verify that (GLL) implies (CLL): if $p_{i} \leq x_{i} \prod_{j \in \Gamma(i)}\left(1-x_{j}\right)$, we can take $y_{i}=\frac{x_{i}}{1-x_{i}}$ (so $\left.1-x_{i}=\frac{1}{1+y_{i}}\right)$ and then use the simple bound

$$
\sum_{\substack{I \subseteq \Gamma^{+}(i) \\ I \in \text { Ind }}} y^{I} \leq \sum_{I \subseteq \Gamma^{+}(i)} y^{I}=\prod_{j \in \Gamma^{+}(i)}\left(1+y_{j}\right) .
$$

On the other hand, Shearer's Lemma (Lemma 5.12) strengthens Theorem 5.40, in the sense that (CLL) implies $p \in \mathcal{S}$. This fact was established by Bissacot et al. [8] by analytic methods that relied on earlier results [17]. In this section we establish this fact by a new proof that is elementary and self-contained.

An algorithmic form of Theorem 5.40 in the variable model was proven by Pegden [33]. In fact, that result is subsumed by the algorithm of Kolipaka and Szegedy in Shearer's setting, since (CLL) implies $p \in \mathcal{S}$. In this section, we prove a new algorithmic form of Theorem 5.40 in the general framework of resampling oracles.

To begin, we establish the following connection between the $y_{i}$ parameters and the $\breve{q}_{S}$ polynomials. For convenience, let us introduce the notation $S^{c}=[n] \backslash S, S+a=S \cup\{a\}$ and $S-a=S \backslash\{a\}$.

Lemma 5.41. Suppose that $p$ satisfies (CLL). Then, for every $S \subseteq[n]$ and $a \in S$, we have

$$
\frac{\breve{q}_{S}}{\breve{q}_{S-a}} \geq \frac{Y_{S^{c}}}{Y_{(S-a)^{c}}}
$$

The proof is in Section 5.7.1 below.

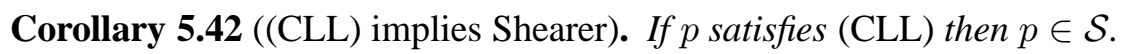

Proof. For any $S \subseteq[n]$, write it as $S=\left\{s_{1}, \ldots, s_{k}\right\}$. Applying Lemma 5.41 repeatedly, we obtain

$$
\frac{\breve{q}_{S}}{\breve{q}_{\emptyset}}=\prod_{i=1}^{k} \frac{\breve{q}_{\left\{s_{1}, \ldots, s_{i}\right\}}}{\breve{q}_{\left\{s_{1}, \ldots, s_{i-1}\right\}}} \geq \prod_{i=1}^{k} \frac{Y_{\left\{s_{1}, \ldots, s_{i}\right\}^{c}}}{Y_{\left\{s_{1}, \ldots, s_{i-1}\right\}^{c}}}=\frac{Y_{S^{c}}}{Y_{[n]}}>0
$$

since $Y_{T}>0$ for all $T \subseteq[n]$ under the (CLL) criterion. Recall that $\breve{q}_{\emptyset}=1$. Hence $\breve{q}_{S}>0$ for all $S \subseteq[n]$, which means that $p$ is in the Shearer region.

Corollary 5.43. If $p$ satisfies (CLL) then $\frac{q_{\{a\}}}{q_{\emptyset}} \leq y_{a}$.

Proof. Lemma 5.41yields $\frac{\breve{q}_{[n]-a}}{\breve{q}_{[n]}} \leq \frac{Y_{([n]-a)^{c}}}{Y_{[n]^{c}}}=1+y_{a}$, so the result follows from Claim 5.25

These corollaries lead to our algorithmic result under the cluster expansion criterion. The following theorem subsumes Theorem 1.3 and adds a statement under the assumption of slack. 
Theorem 5.44. Let $E_{1}, \ldots, E_{n}$ be events and let $p_{i}=\operatorname{Pr}_{\mu}\left[E_{i}\right]$. Suppose that the three subroutines described in Section 1.1.1 exist. If $p$ satisfies (CLL) then, with probability at least $1-e^{-t}$, MaximalSetResample resamples no more than $4\left(\sum_{i=1}^{n} y_{i}\right)\left(\sum_{j=1}^{n} \ln \left(1+y_{j}\right)+1+t\right)$ events.

If (CLL) is satisfied with a slack of $\epsilon \in(0,1)$, i.e., $(1+\epsilon) p_{i} \leq y_{i} / Y_{\Gamma^{+}(i)}$, then with probability at least $1-e^{-t}$, MaximalSetResample resamples no more than $\frac{2}{\epsilon}\left(\sum_{j=1}^{n} \ln \left(1+y_{j}\right)+t\right)$ events.

Proof. The first statement follows directly from Theorem 5.35, since Corollary 5.42 shows that $p \in \mathcal{S}$ and Corollary 5.43 shows that $\frac{q_{\{i\}}}{q_{\emptyset}} \leq y_{i}$. Next assume that (CLL) is satisfied with $\epsilon$ slack. We apply Corollary 5.42 and Corollary 5.43 to the point $p^{\prime}=(1+\epsilon) p$, obtaining that $p^{\prime} \in \mathcal{S}$ and $q_{\{j\}}^{\prime} / q_{\emptyset}^{\prime} \leq y_{j}$, where $q_{S}^{\prime}$ denotes $q_{S}\left(p^{\prime}\right)$. The second statement then follows directly from Corollary 5.31 .

\subsubsection{Proof of Lemma 5.41}

Claim 5.45 (The "fundamental identity" for $Y)$. $Y_{A}=Y_{A-a}+y_{a} Y_{A \backslash \Gamma^{+}(a)}$ for all $a \in A$.

Proof. Every summand $y^{J}$ on the left-hand side either appears in $Y_{A-a}$ if $a \notin J$, or can be written as $y_{a} \cdot y^{B}$ where $B=J \backslash \Gamma^{+}(a)$, in which case it appears as a summand in $y_{a} Y_{A \backslash \Gamma^{+}(a)}$.

Claim 5.46 (Log-subadditivity of $Y$ ). $Y_{A \cup B} \leq Y_{A} \cdot Y_{B}$ for any $A, B \subseteq[n]$.

Proof. It suffices to consider the case that $A$ and $B$ are disjoint, as replacing $B$ with $B \backslash A$ decreases the right-hand side and leaves the left-hand side unchanged. Every summand $y^{J}$ on the left-hand side can be written as $y^{J^{\prime}} \cdot y^{J^{\prime \prime}}$ with $J^{\prime}=J \cap A$ and $J^{\prime \prime}=J \cap B$. The product $y^{J^{\prime}} \cdot y^{J^{\prime \prime}}$ appears as a summand on the right-hand side, and all other summands are non-negative.

Proof (of Lemma 5.41). We proceed by induction on $|S|$. The base case is $S=\{a\}$. In that case we have $\frac{\breve{q}_{\{a\}}}{\breve{q}_{\emptyset}}=\breve{q}_{\{a\}}=1-p_{a}$. On the other hand, by the two claims above and (CLL), we have

$$
Y_{[n]}=Y_{[n]-a}+y_{a} Y_{[n] \backslash \Gamma^{+}(a)} \geq Y_{[n]-a}+p_{a} Y_{\Gamma^{+}(a)} Y_{[n] \backslash \Gamma^{+}(a)} \geq Y_{[n]-a}+p_{a} Y_{[n]} .
$$

Therefore, $\frac{Y_{[n]-a}}{Y_{[n]}} \leq 1-p_{a}$ which proves the base case.

We prove the inductive step by similar manipulations. By Claim 5.13, we have

$$
\frac{\breve{q}_{S}}{\breve{q}_{S-a}}=1-p_{a} \frac{\breve{q}_{S \backslash \Gamma^{+}(a)}}{\breve{q}_{S-a}} .
$$

The inductive hypothesis applied repeatedly to the elements of $S \cap \Gamma(a)$ yields

$$
1-p_{a} \frac{\breve{q}_{S \backslash \Gamma^{+}(a)}}{\breve{q}_{S-a}} \geq 1-p_{a} \frac{Y_{\left(S \backslash \Gamma^{+}(a)\right)^{c}}}{Y_{(S-a)^{c}}}=1-p_{a} \frac{Y_{S^{c} \cup \Gamma^{+}(a)}}{Y_{S^{c}+a}} .
$$

By the two claims above and (CLL), we have

$$
Y_{S^{c}+a}=Y_{S^{c}}+y_{a} Y_{S^{c} \backslash \Gamma^{+}(a)} \geq Y_{S^{c}}+p_{a} Y_{\Gamma^{+}(a)} Y_{S^{c} \backslash \Gamma^{+}(a)} \geq Y_{S^{c}}+p_{a} Y_{S^{c} \cup \Gamma^{+}(a)} .
$$

We conclude that

$$
\frac{\breve{q}_{S}}{\breve{q}_{S-a}} \geq 1-p_{a} \frac{Y_{S^{c} \cup \Gamma^{+}(a)}}{Y_{S^{c}+a}} \geq 1-\frac{Y_{S^{c}+a}-Y_{S^{c}}}{Y_{S^{c}+a}}=\frac{Y_{S^{c}}}{Y_{(S-a)^{c}}}
$$




\subsubsection{Relationship between cluster expansion and stable set sequences}

We remark that the following more general bound holds: For every $J \in$ Ind,

$$
\sum_{\mathcal{I} \in \operatorname{Prop}(J)} p_{\mathcal{I}}=\frac{q_{J}}{q_{\emptyset}} \leq y^{J} .
$$

The equality holds by Lemma 5.27 and the inequality can be derived from Lemma 5.41 as follows:

$$
\frac{q_{J}}{q_{\emptyset}}=\frac{p^{J} \breve{q}_{\left(\Gamma^{+}(J)\right)^{c}}}{\breve{q}_{(\emptyset)^{c}}} \leq p^{J} \frac{Y_{\Gamma^{+}(J)}}{Y_{\emptyset}}=p^{J} Y_{\Gamma^{+}(J)} \leq \prod_{j \in J}\left(p_{j} Y_{\Gamma^{+}(j)}\right) \leq y^{J}
$$

using Claim 5.16 for the first equality, and Claim 5.46 and (CLL) in the last two inequalities.

A direct proof that $\sum_{\mathcal{I} \in \operatorname{Prop}(J)} p_{\mathcal{I}} \leq y^{J}$ can be obtained by an inductive argument similar to the proof of (4) in Section 5.2. An application of Lemma 5.27 then establishes (12). Earlier versions of this paper used this approach to relate the cluster expansion criterion and Shearer's lemma. Our new approach in Corollary 5.42 has the advantage that it does not require the limiting arguments used in Lemma 5.27

\section{Conclusions}

We have shown that the Lovász Local Lemma can be made algorithmic in the abstract framework of resampling oracles. This framework captures the General LLL as well as Shearer's Lemma in the existential sense, and leads to efficient algorithms for the primary examples of probability spaces and events satisfying lopsidependency that have been considered in the literature (as surveyed in [26]).

Our algorithmic form of the General LLL (Theorem 1.2) uses $O\left(\sum_{i=1}^{n} \frac{x_{i}}{1-x_{i}} \sum_{j=1}^{n} \log \frac{1}{1-x_{j}}\right)$ resampling operations, which is roughly quadratically worse than the $\sum_{i=1}^{n} \frac{x_{i}}{1-x_{i}}$ bound of Moser-Tardos [30]. Similarly, our algorithmic result under Shearer's condition (Theorem 5.35) uses $O\left(\sum_{i=1}^{n} \frac{q_{\{i\}}}{q_{\emptyset}} \sum_{j=1}^{n} \ln (1+\right.$ $\left.\frac{q_{\{j\}}}{q_{\emptyset}}\right)$ ) resampling operations, which is roughly quadratically worse than the $\sum_{i=1}^{n} \frac{q_{\{i\}}}{q_{\emptyset}}$ bound of KolipakaSzegedy [24]. Can this quadratic loss be eliminated?

One way to prove that result would be to prove an analog of the witness tree lemma, which is a centerpiece of the Moser-Tardos analysis [30]. The witness tree lemma has other advantages, for example in deriving parallel and deterministic algorithms. Unfortunately, the witness tree lemma is not true in the general setting of resampling oracles (see Appendix A]). It is, however, true in the variable model [30] as well as in the setting of random permutations [21]. Is there a variant of our framework in which the witness tree lemma is true, and which continues to capture the LLL in full generality?

\section{Acknowledgements}

We thank Mohit Singh for discussions at the early stage of this work. We thank David Harris for suggesting the results of Section 3.5, and for discussions relating to Appendix A

\section{References}

[1] Dimitris Achlioptas and Themis Gouleakis. Algorithmic improvements of the Lovász local lemma via cluster expansion. In Proceedings of FSTTCS, 2012. 
[2] Dimitris Achlioptas and Fotis Iliopoulos. Random walks that find perfect objects and the Lovász local lemma. In 55th IEEE Annual Symposium on Foundations of Computer Science, FOCS 2014, Philadelphia, PA, USA, October 18-21, 2014, pages 494-503, 2014.

[3] Dimitris Achlioptas and Fotis Iliopoulos. Random walks that find perfect objects and the Lovász local lemma. CoRR, abs/1406.0242v3, 2015.

[4] Dimitris Achlioptas and Fotis Iliopoulos. Focused stochastic local search and the Lovász local lemma. In Proc. of $27^{\text {th }}$ ACM-SIAM SODA, 2016, to appear.

[5] Saieed Akbari and Alireza Alipour. Multicolored trees in complete graphs. J. Graph Theory, 54:3:221232, 2007.

[6] N. Alon and J. Spencer. The Probabilistic Method. Wiley, 2000.

[7] Noga Alon, Joel Spencer, and Prasad Tetali. Covering with latin transversals. Discrete Applied Mathematics, 57:1:1-10, 1995.

[8] R. Bissacot, R. Fernández, A. Procacci, and B. Scoppola. An improvement of the Lovász local lemma via cluster expansion. Combin. Probab. Comput., 20:709-719, 2011.

[9] Julia Böttcher, Yoshiharu Kohayakawa, and Aldo Procacci. Properly coloured copies and rainbow copies of large graphs with small maximum degree. Random Structures and Algorithms, 40(4), 2012.

[10] Andrei Broder. Generating random spanning trees. In Proceedings of SFCS, pages 442-447, 1989.

[11] Richard A. Brualdi and Susan Hollingsworth. Multicolored trees in complete graphs. J. Combin. Theory Ser. B, 68, 1996.

[12] James M. Carraher, Stephen G. Hartke, and Paul Horn. Edge-disjoint rainbow spanning trees in complete graphs, 2013.

[13] Karthekeyan Chandrasekaran, Navin Goyal, and Bernhard Haeupler. Deterministic algorithms for the Lovász local lemma. SIAM Journal on Computing, 42(6), 2013.

[14] Kai-Min Chung, Seth Pettie, and Hsin-Hao Su. Distributed algorithms for the Lovász local lemma and graph coloring. In Proceedings of PODC, 2014.

[15] Paul Erdös and László Lovász. Problems and results on 3-chromatic hypergraphs and some related questions. In A. Hajnal et al., editor, Infinite and finite sets, volume 10 of Colloquia Mathematica Societatis János Bolyai, pages 609-628. North-Holland, Amsterdam, 1975.

[16] Paul Erdös and Joel Spencer. The Lopsided Lovász Local Lemma and Latin transversals. Discrete Applied Mathematics, 30:151-154, 1991.

[17] R. Fernández and A. Procacci. Cluster expansion for abstract polymer models: New bounds from an old approach. Comm. Math. Phys, 274:123-140, 2007.

[18] Heidi Gebauer, Tibor Szabó, and Gábor Tardos. The local lemma is tight for SAT. In Proceedings of SODA, 2011. 
[19] Ioannis Giotis, Lefteris Kirousis, Kostas I. Psaromiligkos, and Dimitrios M. Thilikos. On the algorithmic Lovász local lemma and acyclic edge coloring. In Proceedings of ANALCO, 2015.

[20] Bernhard Haeupler, Barna Saha, and Aravind Srinivasan. New constructive aspects of the Lovász local lemma. Journal of the ACM, 58(6), 2011.

[21] David G. Harris and Aravind Srinivasan. A constructive algorithm for the Lovász Local Lemma on permutations. In Proceedings of the Twenty-Fifth Annual ACM-SIAM Symposium on Discrete Algorithms, SODA 2014, Portland, Oregon, USA, January 5-7, 2014, pages 907-925, 2014.

[22] Richard Holley. Remarks on the FKG inequalities. Communications in Mathematical Physics, 36:227231, 1974.

[23] Donald E. Knuth. The art of computer programming, Volume 4B (draft, pre-fascicle 6a), 2015. http://www-cs-faculty.stanford.edu/ uno/fasc6a.ps.gz.

[24] Kashyap Kolipaka and Mario Szegedy. Moser and Tardos meet Lovász. In Proceedings of STOC, 2011.

[25] Kashyap Kolipaka, Mario Szegedy, and Yixin Xu. A sharper local lemma with improved applications. In Proceedings of APPROX/RANDOM, 2012.

[26] Lincoln Lu, Austin Mohr, and László Székely. Quest for negative dependency graphs. Recent Advances in Harmonic Analysis and Applications, 25:243-258, 2013.

[27] Austin Mohr. Applications of the lopsided Lovász local lemma regarding hypergraphs. PhD thesis, University of South Carolina, 2013.

[28] Robin Moser. Exact Algorithms for Constraint Satisfaction Problems. PhD thesis, ETH Zürich, 2012.

[29] Robin A. Moser. A constructive proof of the Lovász local lemma. In Proceedings of STOC, 2009.

[30] Robin A. Moser and Gábor Tardos. A constructive proof of the general Lovász Local Lemma. Journal of the ACM, 57(2), 2010.

[31] Sokol Ndreca, Aldo Procacci, and Benedetto Scoppola. Improved bounds on coloring of graphs. European Journal of Combinatorics, 33(4), 2012.

[32] Christos H. Papadimitriou. On the complexity of the parity argument and other inefficient proofs of existence. Journal of Computer and System Sciences, 48:498-532, 1994.

[33] Wesley Pegden. An extension of the Moser-Tardos algorithmic local lemma. SIAM J. Discrete Math, 28:911-917, 2014.

[34] Alexander Schrijver. Combinatorial Optimization: Polyhedra and Efficiency. Springer, 2004.

[35] Alexander D. Scott and Alan D. Sokal. The Repulsive Lattice Gas, the Independent-Set Polynomial, and the Lovász Local Lemma. Journal of Statistical Physics, 118(5):1151-1261, 2005.

[36] James B. Shearer. On a problem of Spencer. Combinatorica, 5(3), 1985.

[37] Joel Spencer. Asymptotic lower bounds for Ramsey functions. Discrete Mathematics, 20:69-76, 1977. 
[38] David E. Woolbright and Hung-Lin Fu. On the existence of rainbows in 1-factorizations of $K_{2 n}$. Journal of Combinatorial Designs, 6:1:1-20, 1998. 


\section{A A counterexample to the witness tree lemma}

A cornerstone of the analysis of Moser and Tardos [30] is the witness tree lemma. It states (roughly) that for any tree of events growing backwards in time from a certain root event $E_{i}$, with the children of each node $E_{i^{\prime}}$ being neighboring events resampled before $E_{i^{\prime}}$, the probability that this tree is consistent with the execution of the algorithm is at most the product of the probabilities of all events in the tree. (We give a more precise statement below.) Extensions of this lemma have been crucial in the work of Kolipaka-Szegedy on algorithmic forms of Shearer's Lemma [24] and work of Harris-Srinivasan on the algorithmic local lemma for permutations [21]. The witness tree lemma leads to somewhat stronger quantitative bounds than the ones we obtain, and it has been also useful for other purposes: derandomization of LLL algorithms [30, 13], parallel algorithms [30, 14], and handling exponentially many events [20]. Therefore, it would be desirable to prove the witness tree lemma in our general framework of resampling oracles.

Unfortunately, this turns out to be impossible. The main purpose of this section is to show that the witness tree lemma is false in the framework of resampling oracles in a strong sense. Whereas in typical scenarios the Moser-Tardos algorithm only requires witness trees of depth $O(\log n)$ with high probability, in the resampling oracle framework the stable set sequences (and an analogous notion of witness trees) can have nearly-linear length with constant probability.

Before we proceed, we define a few notions necessary for the formulation of the witness tree lemma. Our definitions here are natural extensions of the notions from [30] to the setting of resampling oracles.

Definition A.1. Given a lopsided association graph $G$ on vertex set $[n]$, a witness tree is a finite rooted tree $T$, with each vertex $v$ in $T$ given a label $\mathcal{E}_{v} \in[n]$, such that the children of a vertex $v$ receive labels from $\Gamma^{+}\left(\mathcal{E}_{v}\right)$.

Definition A.2. We say that a witness tree $T$ with root $r$ appears in the log of the algorithm, if event $\mathcal{E}_{r}$ is resampled at some point and the tree is produced by the following procedure: process the resampled events from that point backwards, and for each resampled event $j$ such that $j \in \Gamma^{+}\left(\mathcal{E}_{v}\right)$ for some $v$ in the tree, pick such a vertex $v$ of maximum depth in the tree and create a new child $w$ of $v$ with label $\mathcal{E}_{w}=j$.

The witness tree lemma, in various incarnations, states that the probability of a witness tree $T$ appearing in the log of an LLL algorithm is at most $\prod_{v \in T} \operatorname{Pr}\left[E_{\mathcal{E}_{v}}\right]$. We show here that this can be grossly violated in the setting of resampling oracles. Our example actually uses the independent variable setting but resampling oracles different from the natural ones considered by Moser and Tardos.

Example. Consider independent Bernoulli variables $X_{i}, Y_{i}^{j}, Z_{i}$ and $W$ where $1 \leq i \leq k$ and $1 \leq j \leq \ell$. The probability distribution $\mu$ is uniform on the product space of these random variables. Consider the following events:

- $E_{i}=\left\{X_{i}=0\right\}$

- $E_{i}^{j}=\left\{Y_{i}^{j}=0\right\}$

- $E^{\prime}=\{W=1\}$

These events are mutually independent. However, let us consider a dependency graph $G$ where $E_{i} \sim E_{i}^{j}$ for each $1 \leq i \leq k, 1 \leq j \leq \ell$; this is a conservative choice but nevertheless a valid one for our events. (One could also tweak the probability space slightly so that neighboring events are actually dependent.) In any case, $E^{\prime}$ is an isolated vertex in the graph.

We define resampling oracles as follows. In the following, $Q$ describes a fresh new sample of a Bernoulli variable. Only the variables relevant to the respective oracle are listed as arguments. 
- $r_{i}\left(X_{i}\right)=Q$

- $r_{i}^{j}\left(X_{i}, Y_{i}^{j}, Z_{i}\right)=\left(Z_{i}, Q, X_{i}\right)$

- $r^{\prime}\left(W, Z_{1}, \ldots, Z_{k}\right)=\left(Z_{1}, \ldots, Z_{k}, Q\right)$.

Claim A.3. $r_{i}, r_{i}^{j}, r^{\prime}$ are valid resampling oracles for the events $E_{i}, E_{i}^{j}, E^{\prime}$ and the dependency graph $G$.

Proof. $r_{i}$ resamples only the variable $X_{i}$ relevant to event $E_{i}$ and hence cannot cause any other event to occur. Conditioned on $E_{i}=\left\{X_{i}=0\right\}$, it clearly produces the uniform distribution.

$r_{i}^{j}$ switches the variables $X_{i}$ and $Z_{i}$ and thus can cause $E_{i}$ to occur (which is consistent with the dependency graph $G$ ). Conditioned on $E_{i}^{j}=\left\{Y_{i}^{j}=0\right\}$, it makes $Y_{i}^{j}$ uniformly random and preserves a uniform distribution on $\left(X_{i}, Z_{i}\right)$.

$r^{\prime}$ affects the values of $W, Z_{1}, \ldots, Z_{k}$ but no event depends on $Z_{1}, \ldots, Z_{k}$, so $r^{\prime}$ cannot cause any event except $E^{\prime}$ to occur. Conditioned on $E^{\prime}=\{W=1\}$, since $\left(Z_{1}, \ldots, Z_{k}\right)$ are distributed uniformly, it produces again the uniform distribution.

The Moser-Tardos algorithm. First, let us consider the Moser-Tardos algorithm: In the most general form, it resamples in each step an arbitrary occurring event. For concreteness, let's say that the algorithm always resamples the occurring event of minimum index (in some fixed ordering).

Claim A.4. If the Moser-Tardos algorithm considers events in the order $\left(E_{i}, E_{i}^{j}, E^{\prime}\right)$, then at the time it gets to resample $E^{\prime}$, the variables $Z_{1}, \ldots, Z_{k}$ are independent are equal to 1 with probability $1-1 / 2^{\ell+1}$ each.

Proof. Let us fix $i$. Whenever some variable $Y_{i}^{j}$ is initially equal to 0 , we have to resample $E_{i}^{j}$ at some point. However, we only resample $E_{i}^{j}$ if $E_{i}$ does not occur, which means that $X_{i}$ must be 1 at that time. So the resampling oracle $E_{i}^{j}$ forces $Z_{i}$ to be equal to 1 . The only way $Z_{i}$ could remain equal to 0 is that it is initially equal to 0 and none of the events $E_{i}^{j}$ need to be resampled, which happens with probability $1 / 2^{\ell}$. Therefore, when we're done with $E_{i}$ and $E_{i}^{j}$ for $1 \leq j \leq \ell, Z_{i}$ is equal to 0 with probability $1 / 2^{\ell+1}$. This happens independently for each $i$.

Lemma A.5. The probability that the Moser-Tardos algorithm resamples $E^{\prime}$ at least $k$ times in a row is at least $\frac{1}{2}\left(1-\frac{1}{2^{\ell+1}}\right)^{k-1}$.

Proof. By the ordering of events, $E^{\prime}$ is resampled only when all other events have been fixed. Also, resampling $E^{\prime}$ cannot cause any other event, so the algorithm will terminate afterwards. However, as we argued above, when we get to resampling $E^{\prime}$, each variable $Z_{i}$ is equal to 1 independently with probability $1-1 / 2^{\ell+1}$. Considering the resampling oracle $r^{\prime}\left(W, Z_{1}, \ldots, Z_{k}\right)=\left(Z_{1}, \ldots, Z_{k}, Q\right)$, if $W$ as well as all the variables $Z_{i}$ are equal to 1 , it will take at least $k$ resamplings to clear the queue and get a chance to avoid event $E^{\prime}$. This happens with probability $\frac{1}{2}\left(1-\frac{1}{2^{\ell+1}}\right)^{k-1}$.

Let $T$ consist of a path of $k$ vertices labeled $E^{\prime}$. For $k=2^{\ell}$, we conclude that the witness tree $T$ appears with constant probability in the log of the Moser-Tardos algorithm, as opposed to $1 / 2^{k}$ which would follow from the witness tree lemma.

The MaximalSetResample algorithm. A slightly more involved analysis is necessary in the case of MaximalSetResample. By nature of this algorithm, we would resample $E^{\prime}$ "in parallel" with the other events and so the variables evolve somewhat differently. 
Claim A.6. For each $i$ independently, after 2 iterations of the MaximalSetResample algorithm, $Z_{i}=1$ with probability $1-1 / 2^{\ell+1}$. Any further updates of $Z_{i}$ other than those caused by resampling $E^{\prime}$ can only change the variable from 0 to 1 .

Proof. The claim is that unless $Z_{i}=0$ and $Y_{i}^{1}=\ldots,=Y_{i}^{\ell}=1$ initially, in the first two iterations we will possibly resample $E_{i}$ and then one of the events $E_{i}^{j}$, which makes $Z_{i}$ equal to 1 . Any further update to $Z_{i}$ occurs only when $E^{\prime}$ is resampled (which shifts the sequence $\left(Z_{1}, \ldots, Z_{k}\right)$ ) or when $E_{i}^{j}$ is resampled, which makes $Z_{i}$ equal to 1 .

Lemma A.7. The probability that MaximalSetResample resamples $E^{\prime}$ at least $k$ times in a row is at least $\frac{1}{4}\left(1-\frac{1}{2^{\ell+1}}\right)^{k-2}$.

Proof. In the first two iterations, the probability that $E^{\prime}$ is resampled twice is at least $1 / 4$ (the values of $W$ and $Z_{1}$ are initially uniform, and if $Z_{1}$ is updated, it can only increase the probability that we resample $E^{\prime}$ ). Independently, the probability that $Z_{2}=\ldots=Z_{k-1}=1$ after the first two iterations is $\left(1-1 / 2^{\ell+1}\right)^{k-2}$, by the preceding claim. (We are not using $Z_{1}$ which is possibly correlated with the probability of resampling $E^{\prime}$ in the second iteration, and $Z_{k}$ which would be refreshed by this resampling in the second iteration.) If this happens, we will continue to resample $E^{\prime}$ at least $k-2$ additional times, because it will take $k-2$ executions of $r^{\prime}$ before a zero can reach the variable $W$.

Again, consider setting $k=2^{\ell}$. The total number of events is $n=O(k \ell)$, so $\ell=\Theta(\log n)$ and $k=\Theta(n / \log n)$. With constant probability, the witness tree $T$ consisting of a path of $k$ vertices labeled $E^{\prime}$ will appear in the log of MaximalSetResample algorithm. Thus, with constant probability, the algorithm will require a stable set sequence of length at least $k$. 